Martin Setzer

Roland Klopfleisch

Werner Sesselmeier

\title{
Langzeitarbeitslose und Erster Arbeitsmarkt
}




\section{Martin Setzer \\ Roland Klopfleisch \\ Werner Sesselmeier}

\section{Langzeitarbeitslose und Erster Arbeitsmarkt}

Arbeitslosigkeit und insbesondere Langzeitarbeitslosigkeit führen in einer Erwerbsarbeitsgesellschaft zu einer sozialen Exklusion, da die individuellen Chancen und die gesellschaftliche Positionierung jedes einzelnen über die Erwerbsarbeit definiert werden. Deshalb ist es nicht ausreichend, arbeitslose Bürger mit Lohnersatzleistungen $\mathrm{zu}$ versorgen. Beschäftigungspolitik sollte vielmehr darauf abzielen, Arbeitslosen möglichst schnell wieder zu Erwerbsarbeit $\mathrm{zu}$ verhelfen. Dieses Ziel wird in der Arbeit durch eine kombinierte Strategie zu erreichen versucht: Lohnsubventionen für Langzeitarbeitslose werden mit individuell ausgerichteten nicht-materiellen Unterstützungsmaßnahmen gekoppelt. Die damit erreichbaren Angebots- und Nachfragepotentiale werden mit Hilfe der Sozialhilfestatistik und internationaler Vergleiche abgeschätzt.

Martin Setzer, geboren 1965 in Darmstadt, studierte Wirtschaftsingenieurwesen an der TU Darmstadt und war von 1993 bis 1998 wissenschaftlicher Mitarbeiter am Fachgebiet Finanzwissenschaft der TU Darmstadt. Er promovierte 1998.

Roland Klopfleisch, geboren 1966 in Frankfurt am Main, studierte Wirtschaftsingenieurwesen an der TU Darmstadt und ist seit 1994 wissenschaftlicher Mitarbeiter am Fachgebiet Finanzwissenschaft der TU Darmstadt.

Werner Sesselmeier, geboren 1960 in Straubing, studierte Volkswirtschaftslehre an der Universität Regensburg. Seit 1988 ist er wissenschaftlicher Mitarbeiter, seit 1997 Privatdozent am Fachgebiet Finanzwissenschaft der TU Darmstadt. 
Langzeitarbeitslose und Erster Arbeitsmarkt 


\section{Sozialökonomische Schriften}

Herausgegeben von Bert Rürup

Band 16

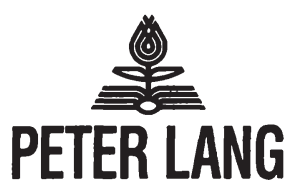

Frankfurt am Main - Berlin · Bern · New York · Paris · Wien 


\section{Martin Setzer \\ Roland Klopfleisch \\ Werner Sesselmeier}

\section{Langzeitarbeitslose und Erster Arbeitsmarkt}

Eine kombinierte Strategie zur Erhöhung der Wiederbeschäftigungschancen

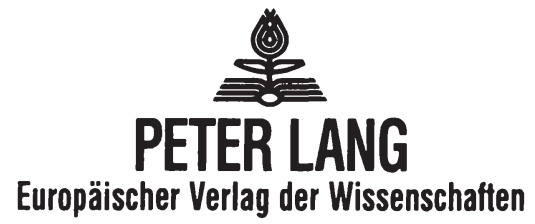


Die Deutsche Bibliothek - CIP-Einheitsaufnahme

Open Access: The online version of this publication is published on www.peterlang.com and www.econstor.eu under the international Creative Commons License CC-BY 4.0. Learn more on how you can use and share this work: http://creativecommons. org/licenses/by/4.0.

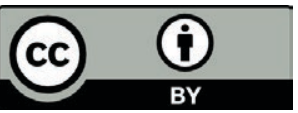

This book is available Open Access thanks to the kind support of ZBW - Leibniz-Informationszentrum Wirtschaft.

Langzeitarbeitslose und Erster Arbeitsmarkt : eine kombinierte Strategie zur Erhöhung der Wiederbeschäftigungschancen / Martin Setzer/Roland Klopfleisch/Werner Sesselmeier. Frankfurt am Main ; Berlin ; Bern ; New York ; Paris ; Wien : Lang, 1999

(Sozialökonomische Schriften ; Bd. 16)

ISBN 3-631-34394-9

Gedruckt auf alterungsbeständigem, säurefreiem Papier.

ISSN 0172-1747

ISBN 3-631-34394-9

ISBN 978-3-631-75277-7 (eBook)

(C) Peter Lang $\mathrm{GmbH}$

Europäischer Verlag der Wissenschaften

Frankfurt am Main 1999

Alle Rechte vorbehalten.

Das Werk einschließlich aller seiner Teile ist urheberrechtlich geschützt. Jede Verwertung außerhalb der engen Grenzen des

Urheberrechtsgesetzes ist ohne Zustimmung des Verlages unzulässig und strafbar. Das gilt insbesondere für Vervielfältigungen, Übersetzungen, Mikroverfilmungen und die Einspeicherung und Verarbeitung in elektronischen Systemen.

Printed in Germany $1234 \quad 67$ 


\section{Vorwort}

Die vorliegende Arbeit entstand in ihrer ersten Version im Auftrag der Stiftung zur Förderung von Wissenschaft und Kultur, Hamburg, und wurde angeregt vom Frankfurter Magistratsmitglied Thomas Koenigs. Aufgabenstellung dieser Studie war die Erarbeitung und konzeptionelle Darstellung von Wiedereinstiegsmöglichkeiten Langzeitarbeitsloser ins Erwerbsleben und sie richtete ihren Fokus auf die regionale Ebene, d.h. hier das Land Hessen. Die Studie wurde am 08.09.1998 der Landespressekonferenz in Wiesbaden vorgestellt und in der regionalen und überregionalen Presse diskutiert.

Der hier vorzustellende Band stellt die inhaltliche Überarbeitung und konzeptionelle Erweiterung der o.g. Studie dar und ist, wenngleich in den empirischen Abschätzungen der Arbeitsangebots- und Arbeitsnachfrageseite auf das Bundesland Hessen konzentriert, auf die überregionale Ebene übertragbar.

Wir danken an dieser Stelle der Stiftung zur Förderung von Wissenschaft und Kultur, Hamburg, für die Unterstützung dieser Studie und Ekkehard Nahm für die wertvolle Hilfe bei den empirischen Recherchen. Wir bedanken uns zudem bei Prof. Dr. Dr. h.c. Bert Rürup, Dr. Henning Stumpp und Dipl-Vw. Jochen Jagob für kritische Hinweise bei der Durchsicht einer früheren Form des Manuskripts. Alle verbleibenden Fehler gehen selbstverständlich ausschließlich zu Lasten der Verfasser.

Martin Setzer, Roland Klopfleisch, Werner Sesselmeier

Darmstadt, November 1998 
Martin Setzer, Roland Klopfleisch and Werner Sesselmeier - 978-3-631-75277-7

Downloaded from PubFactory at 01/11/2019 06:22:32AM

via free access 


\section{Inhalt}

Vorwort

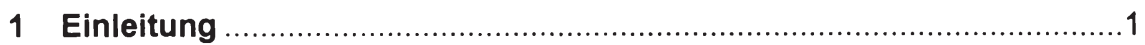

2 Sozioökonomische Rahmenbedingungen und theoretische

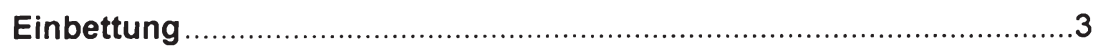

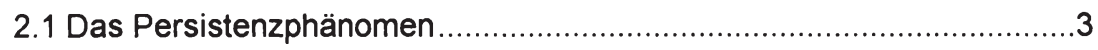

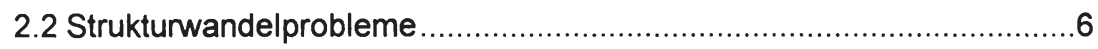

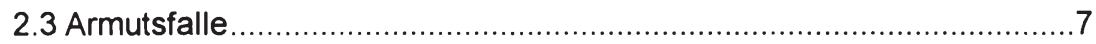

2.4 Beschäftigungspolitische Konsequenzen ................................... 8

3 Überblick, Diskussion und Kritik bestehender Ansätze und Instrumente zur Eingliederung Langzeitarbeitsloser.......................11

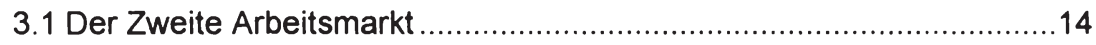

3.1.1 Begriff, Legitimation und Abgrenzung des Zweiten Arbeitsmarktes

3.1.2 Instrumente des Zweiten Arbeitsmarktes.................................15

3.1.3 Ökonomische Analyse des Zweiten Arbeitsmarktes..................17

3.2 Beschäftigungsinitiativen zwischen Erstem und Zweitem Arbeitsmarkt: Kritische Analyse ausgewählter Praxisbeispiele ....................23

3.2.1 Ausgestaltungsformen von Beschäftigungsinitiativen ...............23

3.2.2 Probleme von Beschäftigungsinitiativen ...................................32

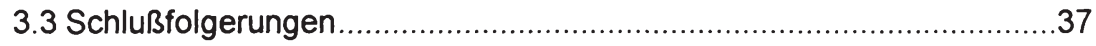


4 Lohnsubventionen zur Integration Langzeitarbeitsloser 40

4.1 Definition und Klassifikation von Lohnsubventionen.

4.2 Auswahl der Subventionsvariablen.

4.2.1 Der Rahmen: Zielführende Integration von Arbeitslosenhilfe und Sozialhilfe

4.2.2 Festlegung der Subventionsparameter

4.3 Modellstruktur und -berechnungen

5 Beratungs-, Betreuungs- und Vermittlungsagentur: „Jobs für Langzeitarbeitslose“

5.1 Grundidee und Rahmenbedingungen

5.2 Aufgaben und Instrumente.

5.2.1 Beratung

5.2.2 Betreuung 63

5.2.3 Vermittlung 64

5.2.4 Akquise und Werbung 64

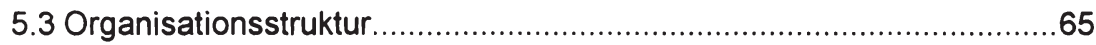

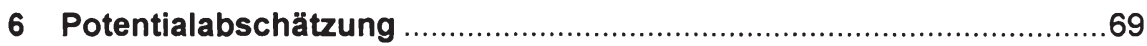

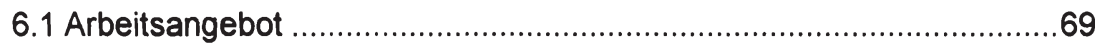

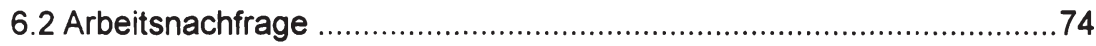

6.2.1 Allgemeine Vorgehensweise ..............................................74

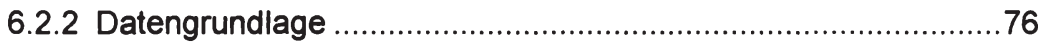

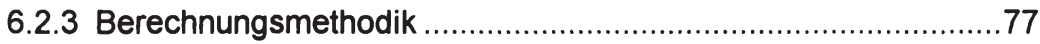

6.2.4 Auswahl der relevanten Berufsgruppen..................................78

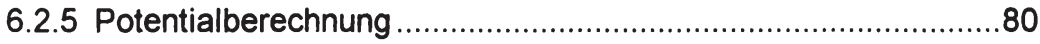

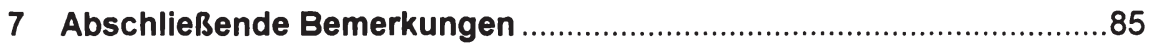

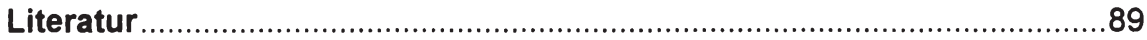

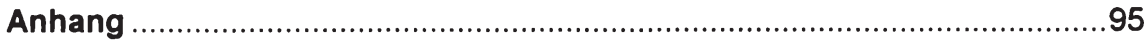




\section{$1 \quad$ Einleitung}

Arbeitslosigkeit und insbesondere Langzeitarbeitslosigkeit führen in einer $\mathrm{Er}$ werbsarbeitsgesellschaft zu einer sozialen Exklusion, da die individuellen Chancen und die gesellschaftliche Positionierung jedes einzelnen über die Erwerbsarbeit definiert werden. Deshalb - und auch im Hinblick auf die Akzeptanz des Sicherungssystems Arbeitslosenversicherung - ist es nicht ausreichend, arbeitslose Bürger mit Lohnersatzleistungen zu versorgen. Vielmehr und dies wurde bereits von Hegel erkannt - kommt es in einer Bürgergesellschaft zur Erhaltung ihres Selbstwertgefühls darauf an, sich das für den Lebensunterhalt benötigte Geld auch verdienen zu können. Beschäftigungspolitik muß folglich darauf abzielen, Arbeitslose möglichst schnell wieder in Erwerbsarbeit zu bringen. Diese Sichtweise ist interessanterweise der Anstoß für das Lohnsubventionsmodell von Phelps (1997), in dem er sogar soweit geht, eine Subvention an die Arbeitgeber zu fordern, um bei den davon begünstigten Arbeitnehmern die Fiktion eines selbst verdienten und das Existenzminimum sichernden Erwerbseinkommens aufrecht zu erhalten. Ein niedrige Markteinkommen ergänzender Transfer hat - unabhängig von seiner konkreten Ausgestaltung - neben dieser gesellschaftspolitischen Aufgabe auch einen erzieherischen Charakter, indem damit Beschäftigung statt Nichtstun subventioniert wird, wie es Hans-Werner Sinn zur Eröffnung der letzten Jahrestagung des Vereins für Socialpolitik etwas provokant formuliert hat. Etwas weniger zugespitzt formuliert, hat der Sozialstaat die Aufgabe, seine Regeln so zu setzen, daß Erwerbsarbeit angeboten, aber auch nachgefragt wird. Dies ist jedoch im besonders von Arbeitslosigkeit betroffenen Bereich der Geringqualifizierten nicht der Fall. Aus diesem Grunde müssen gerade in diesem Bereich die vorhandenen Regeln so angepaßt werden bzw. neue Instrumente so zum Einsatz kommen, daß in Zukunft eine effizientere Allokation der Arbeitskräfte gewährleistet wird. Wie die Erfahrungen aus mittlerweile beschäftigungspolitisch wesentlich erfolgreicheren Nachbarländern zeigen, ist ein Abbau der Arbeitslosigkeit nicht nur mit der Setzung positiver Anreize, wie dies bei Einkommenstransfers der Fall wäre, zu bewerkstelligen. Darüber hinaus sind auch komplementäre negative Anreize notwendig (siehe für einen Überblick Werner 1998). 
Letztendlich haben derartige Maßnahmen zum Ziel, die Eigenverantwortung der Bürger auch und gerade im Rahmen sozialstaatlicher Regelungen wieder stärker zu betonen (Sesselmeier 1998). In diesem Zusammenhang ist schließlich auch darauf hinzuweisen, daß der aktuelle Nobelpreisträger für Wirtschaftswissenschaften, Armatya Sen, auch dafür geehrt wurde, daß er die Realisation von Handlungsfreiheit als eine der wichtigsten Aufgaben von Unterstützungsmaßnahmen sieht und damit - so die königliche Akademie in Stockholm - eine ethische Dimension in die Diskussion über die Ökonomie zurückgebracht hat. Dies korrespondiert nun aber gerade mit dem hier vertretenen Ansatz, daß die Arbeitsmarkt- und Beschäftigungspolitik den Arbeitslosen den Wiedereintritt in den Arbeitsmarkt ermöglichen soll, statt sie nur zu alimentieren.

Mit der vorliegenden Arbeit soll somit ein Beitrag zur laufenden Diskussion um die Möglichkeiten transfergestützter Niedriglohnbereiche geleistet werden, der aufgrund der vorliegenden und auch hier zu präsentierenden Analysen das kürzlich von Vierling (1998) formulierte Motto zugrundeliegt: „Die Frage ist nicht 'ob', sondern 'wie'". Dazu wird in folgenden Schritten vorgegangen:

Als erstes werden die sozioökonomischen Rahmenbedingungen und die theoretischen Grundlagen der Studie sowie die sich aus diesen Überlegungen ergebenden Konsequenzen für die Arbeitsmarkt- und Beschäftigungspolitik dargestellt (Kapitel 2). Daran anschließend und darauf aufbauend werden die gegenwärtigen Konzepte zur Eingliederung von Arbeitslosen in den Ersten wie Zweiten Arbeitsmarkt kritisch analysiert und Schlußfolgerungen für ein eigenes Reformkonzept abgeleitet (Kapitel 3). Im Rahmen dieser Analyse wurde eine umfangreiche Evaluation verschiedener in Hessen ansässiger Beschäftigungsinitiativen durchgeführt, die im Anhang dokumentiert wird. Im dritten Schritt wird ein eigenes Maßnahmenbündel entwickelt und im Hinblick auf eine Umsetzung auf Landesebene konkretisiert (Kapitel 4-6). 


\section{Sozioökonomische Rahmenbedingungen und theoretische Einbettung}

Die Bundesrepublik Deutschland hat nicht nur ein Arbeitslosigkeitsproblem, sondern auch und vor allem ein (Wieder)Beschäftigungsproblem, denn die Entwicklung der Arbeitslosigkeit in den letzten 25 Jahren ist vor allem durch ihren treppenförmigen Anstieg um jeweils etwa 800.000 Personen nach jeder Rezession zu charakterisieren, ohne daß dieser Anstieg bis zur folgenden konjunkturellen Schwächephase wieder abgebaut worden wäre. Insgesamt erscheint diese Arbeitslosigkeit aus einem Zusammenspiel zweier ökonomischer und eines institutionellen Phänomens ableitbar zu sein:

- das Persistenzphänomen,

- Strukturwandelprobleme und

- die sogenannte Armutsfalle.

\subsection{Das Persistenzphänomen}

Theoretisch formuliert kann die in den letzten 25 Jahren und gegenwärtig zu beobachtende Arbeitslosigkeit als persistente oder hysterese Arbeitslosigkeit bezeichnet werden. ${ }^{1}$ Dies bedeutet, daß es keine gleichgewichtige (natürliche) Arbeitslosenquote gibt, die langfristig, also nach vorübergehender Störung, wieder erreicht wird, sondern daß die vorherrschende Arbeitslosenquote ihren eigenen langfristigen Gleichgewichtswert beeinflußt. Bei vollständiger Hysterese läßt sich dann keine eindeutige gleichgewichtige Arbeitslosenquote mehr berechnen. Folglich wird auch nicht das Niveau der Arbeitslosigkeit zur relevanten Größe, sondern die Veränderung der Arbeitslosigkeit unabhängig von ihrem Niveau. Empirisch zeigt sich dies in entsprechenden Schätzungen seit Anfang der sechziger Jahre für die Bundesrepublik Deutschland.

Die Erklärungsansätze für persistente Arbeitslosigkeit, die nicht den Anstieg sondern das Verharren der Arbeitslosigkeit auf einem bestimmten Niveau erklären

Eine ausführliche Darstellung der theoretischen und empirischen Zusammenhänge des Hysteresephänomens mit weiteren Literaturverweisen findet sich in Sesselmeier 1997. 
wollen, können entweder auf der Arbeitsangebotsseite oder auf der Arbeitsnachfrageseite bzw. in deren Zusammenspiel gesehen werden: Im einzelnen sind dies vor allem der Lohnfindungsprozeß und Mismatchgründe.

Zu Beginn der folgenden Argumentationsketten steht allerdings das Humankapital dessen Rolle zunächst erläutert werden soll. Der Begriff des Humankapitals umfaßt alle produktiven Eigenschaften eines Individuums, die es sich durch irgendeine Form von Erziehung oder Ausbildung aneignen kann. Neben Schul- und Berufsausbildung führt auch die Einübung von Eigenschaften wie Lernfähigkeit, Zuverlässigkeit, Teamarbeit oder die Fähigkeit, neue Aufgaben schnell zu übernehmen, zu einer Erhöhung des Humankapitals und damit zu einer Erhöhung der individuellen Produktivität. Diese umfassende Enumeration verdeutlicht, daß das Humankapitalproblem nicht nur die formale Bildung und Qualifikation erfaßt, sondern darüber hinaus auch die Person des Arbeitnehmers sowie dessen Sozialisation umfaßt (extrafunktionale Qualifikation). In der Summe hat das Humankapital sowohl für Arbeitnehmer als auch für Unternehmer eine strategische Bedeutung, die sich auch in den Löhnen widerspiegelt.

Grundlage der theoretischen Erklärungen für Hysterese mit Hilfe von Humankapitalüberlegungen ist die These, daß (Langzeit)Arbeitslosigkeit Dequalifizierungsprozesse und damit eine Veralterung und einen Abbau von $\mathrm{Hu}$ mankapital verursachen kann. Wird ein Arbeitnehmer arbeitslos, kommt es im Verlauf seiner Arbeitslosigkeit zu einer Entwertung des Humankapitals in zweierlei Hinsicht:

1. Die interne Humankapitalentwertung resultiert daraus, daß der Arbeitslose durch mangelnde Übung wichtige Fertigkeiten verlernt und Fachwissen vergißt. Ebenso werden seine Arbeitseinstellung schlechter und die Arbeitsuche mit der Dauer der Arbeitslosigkeit aussichtsloser, da die Suchanstrengungen aufgrund von Entmutigungseffekten sowie sinkenden formalen und informellen Kontakten abnehmen.

2. Die externe Humankapitalentwertung ergibt sich aus der Tatsache, daß gerade konjunkturelle Erholungsphasen durch Neuinvestitionen und damit strukturellen Wandel geprägt werden. In dessen Zuge ändern sich dann Berufsbilder, Ar- 
beitstechniken und Einsatzfelder der Beschäftigten. Der Arbeitslose hat in dieser Phase keine Chance zur Aktualisierung seines Humankapitals.

Folge dieser humankapitaltheoretischen Überlegungen ist, daß im Zeitablauf nicht der gesamte Arbeitslosenpool als Drohpotential für die beschäftigten Arbeitnehmer anzusehen ist, sondern nur ein Teil davon. Diese sogenannte effektive Arbeitslosenquote hat dann Einfluß auf den Lohnbildungsprozeß und die Mismatchproblematik.

Der Einfluß einer persistenten Entwicklung auf den Lohnfindungsprozeß läßt sich dann folgendermaßen modellieren: Nach einem exogenen Schock werden in einer Periode eine bestimmte Anzahl von Arbeitnehmern entlassen, es kommt also zu einem Anstieg der Arbeitslosigkeit. In der Folgeperiode haben diese erst seit kurzem Arbeitslosen noch Einfluß auf die Lohnbildung. Folglich wird es zu eher moderaten Lohnabschlüssen kommen, da die Unternehmen eine Outside-Option haben, die dazu führt, daß - je nach theoretischem Betrachtungswinkel - sowohl die Insider keine hohen Lohnforderungen durchsetzen als auch die Unternehmen geringere Effizienzlöhne zahlen können. Diejenigen Arbeitslosen, die keine Anstellung finden, können in den sich anschließenden Perioden als Langzeitarbeitslose mit entsprechend entwertetem Humankapital interpretiert werden. Aus diesem Grund haben sie keinen Einfluß mehr auf den Lohnbildungsprozeß: Die beschäftigten Insider können wieder höhere Lohnforderungen durchsetzen bzw. die Unternehmen müssen wieder höhere Effizienzlöhne bezahlen, weil die bestehende Arbeitslosigkeit nicht mehr als Drohpotential wirkt. Eine produktivitätsorientierte Lohnpolitik kann das Arbeitslosigkeitsproblem folglich nicht mildern.

Im Hinblick auf die Begründung von Hysterese durch Mismatch geht es schließlich um die Frage, ob und inwieweit die Profile der Arbeitslosen mit den Anforderungen der zu besetzenden Arbeitsplätze übereinstimmen. Im wirtschaftlichen Entwicklungsprozeß fallen regelmäßig unrentable Arbeitsplätze weg und es entstehen neue. Ein beschleunigter Strukturwandel auf dem Arbeitsmarkt kann bei den Erwerbstätigen und Arbeitslosen zu einer Entwertung des Humankapitals führen. Profildiskrepanzen zwischen Arbeitslosen und offenen Stellen, die sich infolge des Humankapitalabbaus bei länger Arbeitslosen ergeben, führen dann zu regionalem und qualifikatorischem Mismatch. Hysterese wird in diesem Argumentationsmu- 
ster empirisch dadurch sichtbar, daß sich die Arbeitslosenquote nach jedem Konjunkturzyklus bei gleicher Vakanzrate erhöht. Dies kann vor allem durch einen im Zeitablauf steigenden Anteil der Langzeitarbeitslosigkeit an der Gesamtarbeitslosigkeit erklärt werden.

Der Problemkern persistenter Arbeitslosigkeit ist also insbesondere in den mangelnden Wiederbeschäftigungschancen der Arbeitslosen zu sehen. Dabei kann diese Arbeitslosigkeit nicht als Marktversagen bezeichnet werden, da der Lohnsatz seiner Allokationsfunktion hinsichtlich der Knappheitsverhältnisse jeweiliger, nach Humankapitalbesitz unterschiedlicher Arbeitnehmergruppen nachkommt.

\subsection{Strukturwandelprobleme}

Das Wiederbeschäftigungsproblem der persistenten Arbeitslosigkeit führt zu der Frage, in welchen Sektoren zusätzliche Arbeitsnachfrage zu erwarten ist (vgl. auch Schettkat 1998). Zunehmende Beschäftigung wird aufgrund der empirischen Situation in beschäftigungspolitisch erfolgreicheren Ländern und der vorliegenden Prognosen nur noch im Dienstleistungssektor zu erwarten sein. Will eine Arbeitsmarkt- und Beschäftigungspolitik erfolgreich sein, also Arbeitslosigkeit abbauen und die Beschäftigung erhöhen, so muß sie diese Zusammenhänge berücksichtigen. Innerhalb dieses Bereiches sind bereits gegenwärtig Angebotslücken in den haushaltsnahen bzw. konsumbezogenen Dienstleistungen feststellbar. Allerdings kann daraus nicht unmittelbar auf eine höhere Beschäftigung geschlossen werden, da gerade in diesen Bereichen andere Preis- und Einkommenselastizitäten der Nachfrage als im sekundären Sektor vorliegen.

Generell kann zunächst eine für reife Volkswirtschaften typische abnehmende Preiselastizität der Nachfrage als Folge sinkender Innovation konstatiert werden. Industriegüter stoßen demnach in industrialisierten Ländern zunehmend auf gesättigte Märkte mit preisunelastischer Nachfrage, so daß der arbeitsplatzsparende Effekt von Produktivitätssteigerungen vorherrscht und zu abnehmender Industriebeschäftigung führt. Daß diese Situation in den OECDLändern dominiert, kann mit einer Fülle von Beispielen aufgezeigt werden: Man denke nur an die überall im Vordergrund stehenden Marketinganstrengungen 
verbunden mit Produktdifferenzierung und Markenbildung, aber auch an die fast flächendeckende Ausstattung der weit überwiegenden Zahl von Haushalten mit langlebigen Konsumgütern. Daneben ist die Entwicklung des Dienstleistungssektors zu betrachten. Die Nachfrage vor allem nach haushaltsnahen Dienstleistungen unterliegt einer hohen Einkommenselastizität. Da der Produktivitätsfortschritt nur den kleineren Teil der von den privaten Haushalten nachgefragten Dienstleistungen berührt und sich die Produktivität zwischen Eigenund Fremdproduktion in diesem Bereich kaum unterscheidet, hängt die makeor-buy-Entscheidung wesentlich von der Differenz zwischen eigenem Nettolohn und dem Preis der Dienstleistung ab. Letzterer wird insbesondere durch das Lohnniveau determiniert.

\subsection{Armutsfalle}

Hinzu kommt als drittes Problem die institutionell bedingte Sozialhilfe- oder Armutsfalle (vgl. Sesselmeier/Klopfleisch/Setzer 1996). Dahinter steht die Ansicht, daß die Lohnspreizung nach unten insbesondere durch Sozialhilferegelungen determiniert wird. Da Langzeitarbeitslosigkeit ein immer häufigerer Grund für den Bezug von Sozialhilfe ist und sich die Sozialhilfeausgaben seit 1985 verdoppelt haben, erscheint es an dieser Stelle angebracht, die externe Effizienz der Sozialhilfe als typisches Beispiel für die institutionell bedingte Anreizproblematik zu analysieren. Externe Effizienz bedeutet dabei, die Wirkungen der Sozialhilfe in bezug auf das Arbeitsangebot zu betrachten, wohingegen interne Effizienz die materielle Absicherung durch die Sozialhilfe zum Gegenstand hat. Allerdings muß festgehalten werden, daß die "Schuld" der Sozialhilfeempfänger an einer eventuell zu geringen Arbeitsneigung nicht ihnen, sondern den institutionellen Regelungen anzulasten ist, die für die betroffenen Personen als exogen gegebene Rahmenbedingungen anzusehen sind, innerhalb derer sie sich nutzenmaximierend verhalten.

Die konsequente Anwendung des Subsidiaritätsprinzips führt zu einer negativen Beeinflussung der externen Effizienz der Sozialhilfe bezüglich des beschäftigungspolitischen Ziels, speziell auf der Angebotsseite, denn eigene Einkünfte werden in nahezu voller Höhe auf die Sozialhilfe angerechnet. Bereits 
Nettoerwerbseinkommen, die nur $25 \%$ des Regelsatzes betragen, werden mit $85 \%$ angerechnet. Dies ist gemäß dem Prinzip der "Hilfe zur Selbsthilfe" zwar durchaus so gedacht, weil die Sozialhilfe nur der Sicherung des Existenzminimums dienen soll und in dem Maße, wie eigenes Einkommen hinzuverdient wird, überflüssig wird. Aus der Sicht des Hilfeempfängers stellt sich die Situation jedoch anders dar: Das verfügbare Einkommen würde sich trotz einer Arbeitsaufnahme praktisch nicht erhöhen, der Quasigrenzsteuersatz betrüge also $85 \%$. Betrachtet man die Entscheidungssituation des Hilfeempfängers, nämlich Arbeit aufzunehmen oder weiter Hilfe zu empfangen, aus mikroökonomischer Sicht, so bewirkt die Arbeitsaufnahme keine nennenswerte Steigerung des Einkommens, wohl aber eine Reduktion der zur Verfügung stehenden Freizeit. Aus diesem Blickwinkel wird der Hilfeempfänger die Aufnahme der Arbeit aus rationalen Gründen ablehnen, man spricht von einer "Armutsfalle" oder "Sozialhilfefalle". Es ist also festzustellen, daß der gegenwärtige Gewährungsmodus der Sozialhilfe unweigerlich starke negative Anreize zur Aufnahme von Arbeit bzw. eine starke externe Ineffizienz mit sich bringt. Somit ist es für den Einzelnen unter den herrschenden Sozialhilfebestimmungen kurzfristig rational, nicht zu arbeiten. Gesamtwirtschaftlich entstehen dadurch zwar auch in kurzfristiger Hinsicht suboptimale Zustände, denn es werden Mittel "verschwendet". Gravierender ist die bestehende Regelung jedoch in langfristiger Perspektive, und dies sowohl für das Individuum als auch für die Gesellschaft. Die kurzfristig rationale Entscheidung, nicht zu arbeiten, führt zu einem zunehmenden Humankapitalabbau bei den betroffenen Personen, der sie im Zeitablauf immer weniger konkurrenzfähig gegenüber den beschäftigten Arbeitnehmern macht. Es kommt also wieder zu typischen Persistenzphänomenen mit der Folge, daß die davon Betroffenen langfristig alimentiert werden müssen.

\subsection{Beschäftigungspolitische Konsequenzen}

Die Überlegungen des voranstehenden Abschnitts sowie vorliegende Analysen zur beschäftigungspolitischen Effektivität und Effizienz aktiver Arbeitsmarktpo- 
litik lassen einige Schlußfolgerungen hinsichtlich Arbeitsangebot, Arbeitsnachfrage und Arbeitsmarktpolitik zu:

- Betrachtet man die Struktur der Arbeitslosen einerseits und die Anforderungen der Arbeitsnachfrage andererseits, so ist festzuhalten, daß für einen Großteil der Langzeitarbeitslosen aufgrund ihrer tatsächlichen oder auch nur vermeintlichen Produktivitätsrückstände keine auskömmlichen Marktlöhne mehr zu erzielen sind. Will man die Entstehung einer working-poorSchicht so weit wie möglich vermeiden, gleichzeitig die betroffenen Personen aber wieder der Erwerbsarbeit zuführen, so wird man nicht um zusätzliche Transferleistungen kommen.

- Vergleicht man die Erwerbsquoten auf internationaler Ebene, so ist für Deutschland ein großer Nachholbedarf im Bereich der binnenmarktorientierten Dienstleistungen zu konstatieren (vgl. Zukunftskommission der Friedrich-Ebert-Stiftung 1998). Dieser Nachholbedarf läßt in Verbindung mit der Tatsache, daß die unteren Lohngruppen in der Industrie kaum mehr besetzt sind (Klös 1997b), nur den einfachen, d.h. wenig Humankapital erfordernden Bereich des binnenmarktorientierten Dienstleistungssektors als zukunftsweisendes Arbeitsplatzreservoir realistisch erscheinen. Diese Ansicht wird zusätzlich dadurch erhärtet, daß die im industriellen Sektor verlorengegangenen Arbeitsplätze aufgrund des technischen Fortschritts aller Wahrscheinlichkeit nach nicht wieder besetzt werden.

- Die bisherige sogenannte mengenorientierte Arbeitsmarktpolitik des Zweiten Arbeitsmarktes kennzeichnet sich in der Hauptsache durch zwei Entwicklungen: Zum einen erfolgt durch die Einrichtung von Beschäftigungsgesellschaften eine Abkopplung vom Ersten Arbeitsmarkt, die zu einer Stigmatisierung der darin Beschäftigten führt, und zum anderen stoßen die Weiterbildungsmaßnahmen an vielfältige individuelle und ökonomische Grenzen. Die Möglichkeit eines Niedriglohnsektors im Ersten Arbeitsmarkt in Verbindung mit lohnunterstützenden Maßnahmen im Sinne einer preisorientierten Arbeitsmarktpolitik erscheint deshalb als der mittel- und langfristig erfolgversprechendere Weg zur Reintegration der (Langzeit)arbeitslosen in die Erwerbsarbeitsgesellschaft. 
Die lohnunterstützenden Transfermaßnahmen können prinzipiell in Form von Steuervergünstigungen, wie sie unter dem Stichwort der negativen Einkommensteuer diskutiert werden, oder als direkte Zuschüsse in Form von Lohnsubventionen erfolgen. Aus organisationspolitischen Überlegungen erscheinen Lohnsubventionen für das hier zu skizzierende Modell als die zu bevorzugende Variante, zumal sie in bezug auf die Zielgruppe als anreizkompatibler betrachtet werden.

Der im Sommer 1998 vorgestellte Gesetzesentwurf der damaligen Bundesregierung steht der voranstehenden Analyse und den daraus abgeleiteten Handlungsoptionen in einigen Punkten entgegen:

- Die Orientierung des Kombilohnes an der Arbeitslosenhilfe und damit am letzten Nettoeinkommen des jeweiligen Arbeitnehmers führt nicht zu einer notwendigen Auffächerung der Lohnstruktur, sondern zementiert viel eher die bestehende. Für den Arbeitnehmer fehlt die Relation zwischen Kombilohn und der neuen Beschäftigung; eine produktivitäts- und knappheitsgerechte Entlohnung ist nicht möglich. Schließlich stellt sich die Frage, welchen Lohn der Arbeitgeber an wen zahlt.

- Die Formulierung, den Kombilohn 'in einem noch zu bestimmenden Niedriglohnsektor einzuführen', ist zumindest mißverständlich:

Meint der Begriff die Lohnspanne, innerhalb derer ein Arbeitslosenhilfeempfänger zum Kombilohnbezug berechtigt ist, so muß diese Lohnspanne konkretisiert werden, um eine mögliche Arbeitsnachfrage zu aktivieren. Dabei ergeben sich notwendigerweise die Probleme, die bereits im vorangegangenen Kritikpunkt aufgezeigt wurden.

Sind mit dem Begriff Niedriglohnsektor jedoch spezifische Arbeitsbereiche gemeint, die vom Gesetzgeber vorgegeben werden sollen und als alleinige Anwendungsfelder des Kombilohnes gelten, so ist eine derartige Regel abzulehnen. Denn dies würde eine ineffektive Kombination eines marktkompatiblen Instruments mit einer dirigistischen Maßnahme bedeuten. Alle positiven und dem Markt zugeschriebenen dynamischen Eigenschaften würden dadurch ausgehebelt. Darüber hinaus würden die Vorteile von Lohnsubven- 
tionen mit Ausrichtung auf den Ersten Arbeitsmarkt verpuffen, da alle Probleme, die aus der mengenorientierten Arbeitsmarktpolitik bekannt sind, auch hier existieren.

- Die Begrenzung des Zielgruppe auf Arbeitslosenhilfeempfänger ist dysfunktional und verletzt das Prinzip horizontaler Gleichbehandlung, da insbesondere langzeitarbeitslose Sozialhilfeempfänger ohne Anspruch auf AFG-Leistungen diskriminiert werden.

- Dauer der Subvention und Befristung des Gesetzes lassen nur eine eingeschränkte Effektivität vermuten, da es zum einen fraglich ist, ob die davon betroffenen Arbeitnehmer in den zwei Jahren so produktiv werden, daß sie auch Arbeitsplätze außerhalb des subventionierten Bereichs finden. Hinzu kommt, daß in den potentiellen Beschäftigungsfeldern personennaher Dienstleistungen nur begrenzte persönliche Produktivitätszuwächse erzielbar sind. Zudem ist die Befristung auf vier Jahre zu kurz, da die Arbeitsplätze in der Hauptsache in Sektoren entstehen werden, in denen es bislang keine oder nur wenig Erfahrung mit solchen Beschäftigungen gibt. Hier ist ein längerer Atem zur Beeinflussung der sozioökonomischen Rahmenbedingungen notwendig.

Die Anreizkompatibilität kann auch durch entsprechenden Druck auf die Zielgruppe unterstützt werden. Dabei geht es allerdings nicht um eine vielfach diskutierte Arbeitspflicht, da diese im Hinblick auf die mit diesem Projekt beabsichtigte Stärkung der Eigenverantwortung dysfunktional wäre, sondern um Überlegungen, die auf eine Absenkung der Transferleistungen mit entsprechenden Aufschlägen bei Arbeitsaufnahme hinauslaufen. Gerade auch in der Kombination verschiedener positiver und negativer Anreizmechanismen liegt der arbeitsmarktpolitische Erfolg in so unterschiedlichen Ländern wie Dänemark oder Großbritannien begründet (vgl. auch Emmerich 1998; Werner 1998). 
Martin Setzer, Roland Klopfleisch and Werner Sesselmeier - 978-3-631-75277-7

Downloaded from PubFactory at 01/11/2019 06:22:32AM

via free access 


\section{3 Überblick, Diskussion und Kritik bestehender Ansätze und Instrumente zur Eingliederung}

\section{Langzeitarbeitsloser}

Der überwiegende Anteil der bestehenden Maßnahmen und Instrumente zur Eingliederung (Langzeit)arbeitsloser kann unter dem Rubrum "mengenorientierte Arbeitsmarktpolitik" zusammengefaßt werden.

Die mengenorientierte Arbeitsmarktpolitik hat in der beschäftigungspolitischen Debatte der letzten Jahre unter dem Stichwort Zweiter Arbeitsmarkt eine beachtliche Konjunktur erlebt, da es als sinnvoller erachtet wird, Arbeit zu finanzieren, als Arbeitslosigkeit zu alimentieren. Neben der Notwendigkeit zur Abgrenzung und Legitimation stellen sich aus ökonomischer Sicht Fragen hinsichtlich der Effekte eines Zweiten Arbeitsmarktes auf Qualifikation, Anreizwirkungen, Allokation und Finanzierung, denen in diesem Kapitel als erstes nachgegangen wird ${ }^{2}$. Insbesondere in der jüngsten Zeit wurden eine Reihe von Institutionen entwickelt, die über die klassischen Instrumente des Zweiten Arbeitsmarktes hinausgehen und eine stärkere Orientierung an dauerhafter, privatwirtschaftlicher Beschäftigung anstreben. Die im weiteren - in Abgrenzung zum Begriff der Beschäftigungsgesellschaft - als Beschäftigungsinitiativen bezeichneten Ansätze werden am Beispiel ausgewählter konkreter Initiativen aus der Praxis diskutiert. Aus beiden Analysesträngen können dann die arbeitsmarktpolitischen Grundlagen für das hier vorzuschlagende Maßnahmenbündel gezogen werden.

2 Eine weitere Auswirkung betrifft die Lohnfindung im Ersten Arbeitsmarkt, die hier aber nicht weiter problematisiert wird; siehe hierzu Ehrlich 1997. 


\subsection{Der Zweite Arbeitsmarkt}

\subsubsection{Begriff, Legitimation und Abgrenzung des Zweiten Arbeitsmarktes}

Der Zweite Arbeitsmarkt ist ein äußert unscharfer Sammelbegriff, für den eine allgemein anerkannte, trennscharfe Definition ${ }^{3}$ nicht existiert, so daß eine Eingrenzung des Begriffs notwendig ist. Der Zweite Arbeitsmarkt bildet einen Teilbereich der aktiven Arbeitsmarktpolitik, worunter in negativer Abgrenzung zur passiven Arbeitsmarktpolitik (Lohnersatzleistungen) Maßnahmen verstanden werden, die die Arbeitsmarktchancen von Arbeitslosen erhöhen, die arbeitsmarktrelevanten Fähigkeiten dieser Personen verbessern und zur Effizienzverbesserung von Arbeitsmärkten beitragen sollen. Aktive arbeitsmarktpolitische Maßnahmen und insbesondere die Maßnahmen des Zweiten Arbeitsmarktes dienen daher der direkten Beeinflussung von Quantität und Struktur des Arbeitskräfteangebots bzw. der Arbeitskräftenachfrage und können deshalb als mengenorientierte Arbeitsmarktpolitik bezeichnet werden.

Der Ausdruck Zweiter Arbeitsmarkt legt ferner eine Abgrenzung zum sogenannten Ersten Arbeitsmarkt nahe. Unter dem Ersten Arbeitsmarkt wird der normale, reguläre, wettbewerbsmäßig strukturierte Arbeitsmarkt verstanden, von dem sich der Zweite Arbeitsmarkt durch die folgenden Merkmale unterscheidet (Bosch u.a. 1994, 50):

- Die öffentliche Förderung zielt auf Beschäftigung und nicht auf Investitionen oder Produkte.

- Die Einstellungskriterien richten sich nach arbeitsmarkt- und sozialpolitischen Zielen.

- Die Beschäftigung ist zeitlich befristet.

- Die ausgeführten Arbeiten müssen einerseits zusätzlich sein, damit nicht reguläre Beschäftigung verdrängt wird, und andererseits die volkswirtschaft-

3 Erstmals erwähnt wurde der Begriff Zweiter Arbeitsmarkt zu Beginn der 80er Jahre in einer Studie der Kammer für soziale Ordnung der evangelischen Kirche in Deutschland 1982. 
liche Wertschöpfung steigern, indem die sozialen Kosten der Arbeitslosigkeit vermindert werden.

- Die Beschäftigung bedarf in einigen Varianten besonderer Trägerinstitutionen (z.B. bei Gesellschaften zur Arbeitsförderung, Beschäftigung und Strukturentwicklung, kurz ABS-Gesellschaften).

Aufgabe des Zweiten Arbeitsmarktes, der somit nur eine ergänzende, subsidiäre Funktion übernimmt, ist die Schaffung von Beschäftigung, die der Erste Arbeitsmarkt nicht in ausreichendem Maße zur Verfügung stellt.

Prüft man die Literatur dahingehend, welche Begründungen für die Etablierung bzw. Enweiterung des Zweiten Arbeitsmarktes vorgebracht werden, so lassen sich im wesentlichen drei Legitimationen unterscheiden:

- Die ökonomische Legitimation, welche den Zweiten Arbeitsmarkt als sozialen Standortfaktor interpretiert, der geeignet ist, infrastrukturelle Voraussetzungen für den privaten Sektor zu verbessern und wichtige Vorleistungen bereitstellen kann;

- die soziale Legitimation, welche die Bedeutung von Erwerbsarbeit für die individuelle soziale und psychologische Wohlfahrt betont;

- die politische Legitimation, welche den Zweiten Arbeitsmarkt für geeignet ansieht, der Erosion der politischen Legitimität des gesellschaftlichen Systems durch anhaltende Massenarbeitslosigkeit entgegenzuwirken.

\subsubsection{Instrumente des Zweiten Arbeitsmarktes}

Die erste Säule des Zweiten Arbeitsmarkts besteht aus den von der Bundesanstalt für Arbeit finanzierten Arbeitsbeschaffungsmaßnahmen ( $A B M)$ gemäß $\S 91 \mathrm{ff}$. AFG. In ABM werden Arbeiten gefördert, die im öffentlichen Interesse liegen und andernfalls nicht oder erst später durchgeführt würden. Während sich in Westdeutschland in der zweiten Hälfte der achtziger Jahre die Teilnehmerzahlen an ABM bereits auf etwa 100.000 erhöhten (ca. 5\% der Arbeitslosen), erfuhren $A B M$ im Zuge der Tansformationskrise Ostdeutschlands mit 466.000 Teilnehmer (ca. 15\% der Arbeitslosen) im Jahr 1992 ihren Höhepunkt. 
1993 sank die Zahl an ABM-Beschäftigten dann wieder auf 288.000, um sich bis 1996 weiter auf 261.000 (6,6\% der Arbeitslosen) zu reduzieren.

Neben den ABM sind auch im Bundessozialhilfegesetz (BSHG) Maßnahmen vorgesehen, die zum Bereich des Zweiten Arbeitsmarktes gerechnet werden können. Es handelt sich hierbei um die sogenannte Hilfe zur Arbeit $(\mathrm{HzA})$ nach $\operatorname{dem} B S H G$, in der Arbeitseinsätze von Sozialhilfeempfängern geregelt werden und die seit den achtziger Jahren von einigen Kommunen praktiziert wird. Bei dem Instrument der $\mathrm{HzA}$ reicht das Spektrum der Bezahlung vom "üblichen Arbeitsentgelt" (Entgeltvariante) bis zur Hilfe zum Lebensunterhalt zuzüglich einer angemessenen Entschädigung für Mehraufwendungen (§19(2) Alt.1,2 BSHG). Bislang war - bei steigender Tendenz - die Mehrzahl der Sozialhilfeempfänger nach der Entgeltvariante beschäftigt ( $57 \%$ von 110.000 Beschäftigten in 1993).

Neben den beiden klassischen Instrumenten werden jedoch auch die wichtigsten arbeitsmarktpolitischen Innovationen der letzten Jahre zum Zweiten Arbeitsmarkt gerechnet: die Sonderarbeitsbeschaffungsmaßnahmen gemäß \$249h AFG und die ABS-Gesellschaften.

Mit dem §249h AFG wurde zunächst zeitlich befristet und allein für Ostdeutschland ein neuer Typ von öffentlich geförderter Beschäftigung eingeführt, der von den meisten Autoren zum Zweiten Arbeitsmarkt gezählt wird, obwohl die Grenze zu Lohnkostenzuschüssen fließend ist ${ }^{4}$. Seit 1993 können in Ostdeutschland Arbeitsämter den in der Vorschrift definierten Personenkreis in Aufgaben der Umweltverbesserung, der sozialen Dienste und der Jugendhilfe vermitteln und für eine Dauer von max. 36 Monaten einen Lohnkostenzuschuß in Höhe der durchschnittlichen monatlichen Aufwendungen an Arbeitslosengeld und Arbeitslosenhilfe einschließlich der Beiträge zur Renten- und Krankenversicherung gewähren (ca. 30-40\% der gesamten Maßnahmekosten) ${ }^{5} .1996$ waren in Westdeutschland 6.300 und in Ostdeutschland 86.300 Personen mit produktiven Lohnkostenzuschüssen beschäftigt.

$4 \mathrm{Zu}$ Eingrenzungsproblemen sowie Modellen zur Ausgestaltung des 2. Arbeitsmarktes siehe bspw. Kress 1994, 3ff.

5 Inzwischen wurde der \$249h AFG mehrmals verlängert, die Tätigkeitsbereiche erweitert und als §242s AFG auf Westdeutschland übertragen. Vgl. z.B. Sperling 1994. 
Dem Zweiten Arbeitsmarkt zuzurechnen sind ferner ABS-Gesellschaften. Daß ABS-Gesellschaften extra erwähnt werden, mag zunächst verwundern, da in innen Beschäftigung meist durch Lohnkostenzuschüsse gemäß $\$ 249 \mathrm{~h}$ AFG oder ABM gefördert wird, jedoch stellt insbesondere der institutionelle Rahmen der ABS-Gesellschaften in Ostdeutschland eine vielbeachtete Neuerung dar, die bei einem weiteren Ausbau des Zweiten Arbeitsmarktes Vorbildfunktion haben könnte. Mit den ABS-Gesellschaften wurde ein Netz neuer Träger geschaffen: Gesellschaften zur Arbeitsförderung, Beschäftigung und Strukturentwicklung. Diese zunächst zeitlich befristet ins Leben gerufenen Institutionen sollen primär Strukturprobleme bekämpfen und werden zu großen Teilen aus Mitteln der Bundesanstalt für Arbeit finanziert. Die ABS-Gesellschaften, die nicht (primär) auf Gewinnerzielung ausgerichtet sind, knüpfen häufig an den Beschäftigungsstrukturen von Altunternehmen und Verwaltungen an, wodurch sich auch in Ostdeutschland sehr häufig die Einbindung in kollektivrechtliche Vereinbarungen zwischen Unternehmen, der Treuhand und Gewerkschaften erga $^{6}$. Hinsichtlich der quantitativen Bedeutung ergaben Umfragen des IAB etwa 400 ABS-Gesellschaften in Ostdeutschland mit rund 110.000 Teilnehmern (1994) ${ }^{7}$.

\subsection{3 Ökonomische Analyse des Zweiten Arbeitsmarktes}

\subsubsection{Anreizaspekte}

Bei der Analyse des Zweiten Arbeitsmarktes kann man zwei Anreizprobleme unterscheiden. Von Kritikern wird in erster Linie das vertikale Anreizproblem hervorgehoben: Den Teilnehmern würde durch die Bereitstellung gut bezahlter Arbeitsplätze im Zweiten Arbeitsmarkt der individuelle Anreiz genommen (oder zumindest verringert), eine reguläre Beschäftigung aufzunehmen. Die öffentlich geförderte Beschäftigung würde somit als regulärer Arbeitsplatz betrachtet, und die Suchintensität nach einer unsubventionierten Stelle auf dem Ersten

6 Auf die Unterschiede zwischen ABS-Gesellschaften und in Westdeutschland praktizierten Formen der Beschäftigungsgesellschaften soll hier nicht eingegangen werden.

7 Das im Juni 1996 vorgelegte Arbeitsförderungs-Reformgesetz (AFRG) weist im 6. Kapitel die wesentlichen Instrumente des 2 . Arbeitsmarktes erstmals zusammen aus. 
Arbeitsmarkt würde abnehmen. Neben diesem Aspekt gibt es jedoch noch ein weiteres Anreizproblem, da der Lohnsatz des Zweiten Arbeitsmarktes auch eine Anreiz- und Motivationsfunktion für die innerhalb des Zweiten Arbeitsmarktes Beschäftigten erfüllen muß (horizontales Anreizprob/em).

Beide Anreizprobleme stehen sich nun hinsichtlich ihrer Wirkungsketten und Anforderungen an die Lohnhöhe diametral gegenüber. Das vertikale Anreizproblem ist um so geringer, je niedriger die Entlohnung auf dem Zweiten Arbeitsmarkt im Vergleich zum ersten ist, während das horizontale Anreizproblem um so geringer ist, je höher die Entlohnung liegt. Empirische Untersuchungen über die Wirkungen der Arbeitslosenunterstützungssysteme liefern in Verbindung mit Teilnehmerbefragungen insgesamt eher Argumente dafür, daß der Entlohnungs- bzw. Unterstützungshöhe nicht die vorrangige Bedeutung bei der Entscheidung, einen Arbeitsplatz anzunehmen, zukommt. Beispielsweise erklärten bei einer Untersuchung $72 \%$ der Befragten, ihre ABM sofort zu verlassen, wenn sich innen ein fester Arbeitsplatz böte (Miethe/Brödnow 1993). Hinsichtlich der horizontalen Anreizprobleme zeigen experimentelle und empirische Studien die Bedeutung von Lohndifferenzierung für die Motivation auch im Zweiten Arbeitsmarkt. So wird Z.B. der Erhalt individueller Qualifikationen bei der Mehraufwandsvariante deutlich negativer gesehen, als bei der Entgeltvariante. Vermittlungen in ein reguläres Arbeitsverhältnis sind bei Maßnahmen der Entgeltvariante ebenfalls deutlich höher als bei der Mehraufwandsvariante. Folglich sollte das horizontale Anreizproblem unter Beachtung der vertikalen Beschränkungen prioritär behandelt werden.

\subsubsection{Allokationseffekte}

In der Diskussion über den Zweiten Arbeitsmarkt spielen unter allokativen Aspekten einerseits Verdrängungs- und Mitnahmeeffekte und andererseits Fehlallokationen und damit die Verschwendung knapper Ressourcen eine gewichtige Rolle.

Generell wird unter Verdrängungseffekten die Verdrängung nicht-subventionierter Arbeit auf dem ersten durch subventionierte Arbeit auf dem Zweiten Arbeitsmarkt verstanden. Einem „Drehtüreneffekt" gleich wird vermutet, daß re- 
guläre Arbeitsplätze im gleichen Umfang verloren gehen wie öffentlich subventionierte Stellen geschaffen werden. Mitnahmeeffekte treten ein, wenn durch die Subventionierung Arbeitsplätze gefördert werden, die auch ohne diese Zahlung eingerichtet worden wären oder die zu einem späteren Zeitpunkt geplant waren. Staatliche Zuschüsse werden in diesen Fällen als "windfall profits" von den Trägern eingestrichen, und die Beschäftigungswirkung der staatlichen Leistungen wird durch dieses Verhalten der Unternehmen reduziert.

Um Verdrängungs- und Mitnahmeeffekte abschätzen zu können, müßte allerdings ein Referenzszenario ursprünglich intendierter Handlungen angenommen werden, welches sich ohne die entsprechenden arbeitsmarktpolitischen Instrumente ergeben hätte. Die Gestaltungsspielräume bei solchen Vergleichen sind allerdings so groß, daß sie je nach Interessenlage geringe oder hohe Effekte zum Ergebnis haben. In einer Studie der OECD werden für eine Reihe von Industrieländern bedeutende Effekte festgestellt: Das irische „Employment Incentive"-Programm führte zu Verdrängungseffekten in der Größenordnung von 95\%; im "Jobstart"-Programm in Österreich machten die Mitnahmeeffekte zwischen $67 \%$ und $79 \%$ aus und bei den Einstellungssubventionen in den Niederlanden betrugen die Verdrängungs- und Mitnahmeeffekte zwischen 76 und $89 \%$ (OECD 1993). Während in den achtziger Jahren für westdeutsche Kommunen bedeutende Verdrängungseffekte nachgewiesen wurden (z.B. im Büround Verwaltungsbereich Hamburgs), kommen die meisten Studien für Ostdeutschland zu eher geringen Verdrängungs- und Mitnahmeeffekten. Außerdem können derartige Effekte durch institutionelle Arrangements verhindert werden. So gilt für ABM das sogenannte „Zusätzlichkeitskriterium“ (§91 Abs. 2 $A F G)$, nach dem nur Arbeiten gefördert werden dürfen, die „sonst nicht oder erst zu einem späteren Zeitpunkt" durchgeführt worden wären. Zudem ist es inzwischen auch gängige Praxis der Arbeitsämter, vor der Durchführung einer ABM z.B. von den Handwerkskammern eine Unbedenklichkeitsbescheinigung einzuholen. Insofern ist es nicht verwunderlich, daß das IAB zu dem Ergebnis kommt, daß Verdrängungs- und Mitnahmeeffekte durch ABM in Ostdeutsch- 
land in nennenswertem, aber insgesamt geringem Umfang (Spitznagel 1992) ${ }^{8}$ zu verzeichnen waren.

Ein weiterer Problembereich liegt in der vermuteten Verschwendung von Ressourcen durch kostspielige Projekte im Zweiten Arbeitsmarkt mit geringen positiven Wirkungen. Trotz der wenigen Studien auf diesem Gebiet darf aber in Anbetracht der bisherigen Ergebnisse bezweifelt werden, daß in großem Umfang öffentliche Gelder verschwendet werden. ABM werden in den neuen Ländern überwiegend investiv eingesetzt und primär in solchen Bereichen, die eine hohe Förderpriorität besitzen (Umweltsanierung, Infrastrukturmaßnahmen). Empirische Hinweise auf positive strukturelle Wirkungen konnten in einer Untersuchung von ABS-Gesellschaften in Sachsen-Anhalt nachgewiesen werden: ABS-Gesellschaften erbrachten nach dieser Studie wichtige Vorleistungen für private Kleinunternehmen. Langfristig positive Effekte zeigen sich auch in international vergleichenden Studien, die feststellen, daß Länder mit einem hohen Aktivitätsniveau der aktiven Arbeitsmarktpolitik c.p. eine niedrigere Arbeitslosigkeit aufweisen. Eine Verlagerung von Mitteln von der passiven zugunsten einer aktiveren Verwendung scheint - unabhängig von einer Bewertung des Zweiten Arbeitsmarktes - anstrebenswert.

\subsubsection{Qualifizierungsaspekte}

Auf der theoretischen Grundlage der in Kapitel 1 beschriebenen Situation hystereser Arbeitslosigkeit können die durch einen Zweiten Arbeitsmarkt erbrachten Qualifizierungsleistungen systemimmanente Koordinationsprobleme des Arbeitsmarktes abmildern. Von den Maßnahmen des Zweiten Arbeitsmarktes könnten sowohl positive mikro- als auch makroökonomische Wirkungen ausgehen. Mikroökonomisch könnte der Zweite Arbeitsmarkt ein Marktversagen bei der Vermittlung allgemeinen Humankapitals abmildern, während er makroökonomisch durch eine Erhöhung der effektiven Arbeitslosigkeit zu einer besseren Allokation auf dem Arbeitsmarkt beitragen könnte.

8 Bei dieser Einschätzung ist allerdings zu berücksichtigen, daß aufgrund des bisher mässig entwickelten Ersten Arbeitsmarktes in Ostdeutschland kaum Verdrängungseffekte zu erwarten sind. 
In Anbetracht der vorliegenden empirischen Ergebnisse sind jedoch Zweifel an den theoretisch positiven Wirkungen angebracht: Ein Großteil der Maßnahmeteilnehmer wurde nicht qualifikationsadäquat eingesetzt und in den Maßnahmen eher unterfordert. Nur in wenigen Fällen vermittelten die untersuchten Maßnahmen Qualifikationen, die die Eingliederung in reguläre Beschäftigungsverhältnisse erleichtern konnten. Dies zeigt sich auch - neben anderen Ursachen - an der geringen Zahl von Übergängen in reguläre Beschäftigungsverhältnisse (vgl. Ehrlich 1997, 220). Sowohl unter dem Gesichtspunkt der Erhaltung und Erweiterung vorhandener beruflicher Qualifikationen als auch unter dem Aspekt der Aneignung neuer, knappheitsgerechter beruflicher Qualifikationen sind die zuvor geschilderten Maßnahmen eher negativ zu beurteilen. Dieser Befund bedeutet allerdings auch, daß die soziale Legitimation des Zweiten Arbeitsmarktes nur sehr eingeschränkt als erfüllt angesehen werden kann.

\subsubsection{Finanzierungsaspekte}

Bei der ökonomischen Betrachtung ist abschließend nach fiskalischen Belastungen durch einen Zweiten Arbeitsmarkt zu fragen. Empirische Studien verwenden hierzu Refinanzierungsrechnungen, in denen untersucht wird, in welchem Verhältnis die Kosten der verschiedenen Maßnahmen zu den Kosten stehen, die andernfalls aus der Arbeitslosigkeit entstanden wären. Hierbei ist zu beachten, daß eine solchermaßen fiktive gesamtfiskalische Sicht ausblendet, daß für einzelne Haushalte (Bund, Bundesanstalt für Arbeit, Kommunen etc.) die Be- und Entlastungen höchst unterschiedlich sein können, die hier nicht betrachtet werden.

Für ABM sind eine Vielzahl von Schätzungen unternommen worden, deren Angaben zu Refinanzierungsquoten zwischen 60\% und 95\% schwanken. Stellt man diese Untersuchungen nur für den Haushalt der Bundesanstalt für Arbeit an, so liegt die Refinanzierungsquote zwischen 46 und $66 \%$, wobei die höheren Zahlen durch Berücksichtigung indirekter Wirkungen (z.B. Vorleistungen, Multiplikatoreffekte) von ABM entstehen. Für Sonderarbeitsbeschaffungsmaßnahmen nach $\$ 249 \mathrm{~h}$ AFG, die zumindest der Intention nach für den Haushalt 
der Bundesanstalt für Arbeit ein kostenneutrales Instrument der Arbeitsmarktpolitik darstellen, liegen ebenso wie für ABS-Gesellschaften noch keine gesamtfiskalischen Kostenanalysen vor. Neuere Studien zur Wirksamkeit des Instruments "Hilfe zur Arbeit" nach dem BSHG zeigen, daß ab dem zweiten Jahr sozialversicherungspflichtiger Beschäftigung eine Amortisation der Maßnahmekosten einsetzen kann.

Diese günstigen Finanzierungseigenschaften des Zweiten Arbeitsmarktes beruhen allerdings teilweise auf impliziten Annahmen, welche den dargestellten Berechnungen zugrunde liegen. Zum einen werden reale Belastungen durch die Maßnahmekosten mit hypothetischen Entlastungen verglichen, die nur eintreten, wenn sich der Status des Teilnehmers für die Dauer der Betrachtung nicht ändern würde. Weiterhin sind die angenommenen Multiplikatorwirkungen (zusätzliche Nachfrage durch die Erhöhung der Kaufkraft) sehr umstritten und keineswegs nachweisbar. In jedem Fall aber gelten die obigen Abschätzungen nur für den bisherigen - begrenzten - Umfang des Zweiten Arbeitsmarktes.

Als Fazit der theoretischen und empirischen Betrachtungen zeigt sich, daß die Etablierung eines Zweiten Arbeitsmarktes über den bisherigen, begrenzten und stark durch die Sondersituation der neuen Bundesländer geprägten Rahmen hinaus kritisch zu bewerten ist. Zwar kann der ökonomische Gesamteffekt eines Zweiten Arbeitsmarktes nicht abschließend bewertet werden, da die untersuchten Wirkungsketten zum Teil gegenläufige Folgen haben. Die niedrigen Übergangsquoten, Drehtüreffekte und die Gefahr der Abspaltung von den Bedingungen des Ersten Arbeitsmarktes zeigen jedoch, daß sich wirtschaftspolitische Maßnahmen auf letzteren konzentrieren sollten. 


\subsection{Beschäftigungsinitiativen zwischen Erstem und Zweitem Arbeitsmarkt: Kritische Analyse ausgewählter Praxisbeispiele}

\subsubsection{Ausgestaltungsformen von Beschäftigungsinitiativen}

\subsubsection{Begriffsdefinition}

Unter dem Begriff Beschaftigungsinitiative wird in dieser Studie eine breite Palette von zumeist kommunalen Aktivitäten hinsichtlich der (Wieder-) Eingliederung von schwer vermittelbaren Arbeitsuchenden verstanden ${ }^{9}$. Die untersuchten Ausgestaltungsformen umfassen im einzelnen ${ }^{10}$.

- Sozialamtsreferate

- Referat Fallmanagement im Sozialamt Offenbach

- aus dem Sozialamt ausgegliederte, eigens zur Beschäftigungsförderung eingerichtete Ämter

- Amt für Arbeitsförderung und Statistik in Offenbach

- von der Kommune beauftragte Beschäftigungsgesellschaften

- Offenbach: Gemeinnützige Offenbacher Ausbildungs- und Beschäftigungsgesellschaft (GOAB)

- Bielefeld: Regionale Personalentwicklungsgesellschaft mbH (REGE)

- Hofheim: Selbsthilfe im Taunus (SiT)

- Internationaler Bund (IB), tätig in mehr als 300 Orten bundesweit, hier: Darmstadt

- Duisburg: START Zeitarbeit NRW, 33 Standorte in Nordrhein-Westfalen

- einzelne (Pilot-)Projekte

- Projekt 1.000 Ressourcen (Kooperation REGE mbH und INITA, vgl. Anhang)

- Projekt WORK-IN (SiT)

9 Als schwer vermittelbar gelten Personen, die mehrere vermittlungshemmende Merkmale aufweisen, die eine Arbeitsaufnahme erheblich behindern. Sie zählen in der Regel zur Gruppe der Sozialhilfebeziehenden bzw. Langzeitarbeitslosen.

10 Die detaillierte Untersuchung und Angaben zu den einzelnen Beschäftigungsinitiativen befinden sich im Anhang dieser Studie. 
Nicht zu den in diesem Abschnitt untersuchten Beschäftigungsinitiativen zählen all jene Beschäftigungsgesellschaften, deren vornehmliches Ziel die Beschäftigung auf dem Zweiten Arbeitsmarkt und nicht die Vermittlung in den Ersten Arbeitsmarkt ist.

\subsubsection{Abgrenzung und Auswahl der Untersuchungsobjekte}

Die Anzahl der Beschäftigungsinitiativen in Deutschland ist nicht zuletzt aufgrund der weit gefaßten Definition und der engen Vernetzung untereinander kaum feststellbar. Anfang 1998 zählte der Verein "Wirtschaftsförderung Region Frankfurt / Rhein-Main" allein in der Rhein-Main-Region 112 Projekte mit dem Oberziel "Vermittlung in den Ersten Arbeitsmarkt", von denen sich immerhin siebzehn ausschließlich mit dem Schwerpunkt "Einstieg in den Ersten Arbeitsmarkt" befaßten, eines unter innen das Projekt WORK-IN der SiT (vgl. Anhang, SiT). Im Rahmen dieser Erhebung wurden 75 Kommunen, 35 Beschäftigungsgesellschaften und acht Arbeitsämter befragt (vgl. Wirtschaftsförderung Region Frankfurt/ Rhein-Main e.V. 1998, 3f). Eine Hochrechnung dieser Zahlen auf Landes- bzw. Bundesebene ist aufgrund der erheblichen Unterschiede in den Aktivitäten der einzelnen Regionen wenig sinnvoll ${ }^{11}$. Aus der Vielzahl an Beschäftigungsinitiativen wurden im Rahmen dieser Studie sechs näher untersucht. Die getroffene Auswahl soll einen Überblick über die verschiedenen Ausgestaltungsformen von Beschäftigungsinitiativen auf Bundesebene ermöglichen. Ihr Ergebnis stellt ein repräsentatives, jedoch kein vollständiges Abbild dar. Im folgenden wird kurz auf die Gründe der getroffenen Auswahl eingegangen.

Die GOAB (Gemeinnützige Offenbacher Ausbildungs- und Beschäftigungsgesellschaft) wurde aufgrund ihrer langjährigen Erfahrungen und ihrer mittlerweile überregionalen Tätigkeit im Rahmen ihres Projektes 'matchwork' ausgewählt (Aus- und Weiterbildung von Arbeitsvermittlern). Sie finanziert sich aus mehreren Quellen. Der Anteil der Erlöse aus ihrem Eigenbetrieb an der Finanzierung

11 Böckmann-Schewe/Röhrig $(1997,36)$ liefern eine tabellarische Übersicht aller Landesmittel zur "Hilfe zur Arbeit", welche einen Anhaltspunkt bezüglich der unterschiedlichen Gewichtung der Länderaktivitäten bietet. 
wächst beständig. Aufgrund der engen Verbindungen zum Sozialamt Offenbach fand auch das dortige Referat Fallmanagement Aufnahme in die Untersuchungen. Während die GOAB für die Vermittlung in Arbeit zuständig ist - verbunden mit allen hierfür als notwendig erachteten qualifizierenden und stabilisierenden Maßnahmen -, berät das Referat Fallmanagement eine ausgesuchte Klientel in Fragen spezieller Vermittlungshemmnisse (innerfamiliäre Situation, Kinderbetreuungssituation, Wohnsituation, wirtschaftliche Situation). Es übt dabei stellvertretend für die jeweiligen Sachbearbeiter einen Teil der originären Sozialamtsaufgaben aus, für die im Tagesgeschäft aufgrund der großen Zahl zu bearbeitender Fälle keine Zeit verbleibt.

Ebenfalls wegen ihrer Verbindungen zur GOAB wurde die SiT (Selbsthilfe im Taunus) untersucht. Sie baut zur Zeit in Kooperation mit der GOAB und dem Arbeitsamt Frankfurt die Initiative Vermittlung in Arbeit - Frankfurt (VIA) auf, die sich verstärkt um die Eingliederung von Arbeitslosen bemühen soll. Entstanden mit dem Schwerpunkt der Suchtberatung, besitzt die SiT über ihr Projekt WORK-IN Erfahrungen im Bereich des eingliederungsorientierten Arbeitnehmerverleihs, ähnlich der START Zeitarbeit gGmbH (Nordrhein-Westfalen).

Ebenfalls in Nordrhein-Westfalen (Bielefeld) angesiedelt ist die REGE mbH, die in Zusammenarbeit mit dem Institut für angewandte Psychologie, Transaktionsanalyse und Erwachsenenbildung (INITA) aus Hannover das Projekt 1.000 Ressourcen durchführt. Während sich START aus seinen Erlösen finanziert und relativ leicht vermittelbare Arbeitslose - der Anteil an Sozialhilfeempfängern liegt bei etwa $5 \%$ - unter Vertrag nimmt, wird das Projekt 1.000 Ressourcen aus den verschiedensten Finanzquellen bezuschußt und bemüht sich um die Vermittlung eines repräsentativen Querschnitts an arbeitsfähigen Sozialhilfeempfängern der Stadt Bielefeld.

Schließlich wurde der Internationale Bund berücksichtigt, da er aufgrund seiner vielfältigen Aufgabenbereiche und seiner weiten nationalen wie internationalen Vernetzung eine Sonderstellung unter den Beschäftigungsinitiativen einnimmt. Die Auswahl des Standortes Darmstadt fand aus regionalen Gründen statt.

Auch in anderen Industrienationen sind Bemühungen $\mathrm{zu}$ beobachten, (Langzeit-)Arbeitslosigkeit mit ähnlichen Instrumenten zu bekämpfen. Bezogen 
auf Deutschland darf das niederländische bureau maatwerk nicht unerwähnt bleiben, welches 1996 durch seine Vermittlungstätigkeit in Hamburg Aufmerksamkeit erregte. Die langjährig erprobte Methode der paßgenauen Vermittlung, gekoppelt mit den Instrumenten der Beratung vor sowie Betreuung nach der Arbeitsvermittlung und der intensiven Kontaktpflege mit ansässigen Unternehmen durch gut ausgebildete Arbeitsvermittler, stellt die Basis vieler hiesiger Beschäftigungsinitiativen dar. Das Erfolgskriterium der Arbeit von maatwerk ist die Erwerbssituation der vermittelten Personen sechs Monate nach der Vermittlung. Trotz der allseits anerkannten Qualität der Vermittlungsmethode von maatwerk wurde in jüngster Zeit vermehrt Kritik geäußert: Die ausschließliche Konzentration auf das o.g. Erfolgskriterium, gekoppelt mit einer Projektbefristung auf zumeist ein Jahr, entlaste die Kommunen nur kurzfristig. Nur langjährige Bemühungen, welche ebenso Angebote für zunächst nicht Vermittelbare beinhalten, schonen kommunale Haushalte dauerhaft.

Alle bisher erwähnten Initiativen waren oder sind in Großstädten tätig. Auf Landkreisebene existieren jedoch ebenfalls Beschäftigungsinitiativen, wobei hier das Netzwerk Lippe (Detmold) sowie das Zentrum Arbeit und Umwelt (ZAUG) im Landkreis Gießen erwähnenswert sind ${ }^{12}$.

\subsubsection{Zielgruppen und Arbeitsweisen}

Je nach Ausgestaltungsform ist der Anteil an Sozialhilfeempfängern von Initiative zu Initiative unterschiedlich hoch: Während das Referat Fallmanagement ausschließlich Sozialhilfeempfänger vermittelt, beträgt beispielsweise der Anteil der Sozialhilfeempfänger bei START Zeitarbeit NRW lediglich 5,3\% (1997). Das Kriterium des Sozialhilfebezugs ist jedoch nicht immer gleichzusetzen mit der Schwere der Vermittelbarkeit. Alle Initiativen versuchen, einen

12 Darüber hinaus existieren seit einigen Jahren zahlreiche Beschäftigungsbetriebe des Zweiten Arbeitsmarktes, welche im Zuge ihrer Arbeit ebenfalls Vermittlungen in den Ersten Arbeitsmarkt zu verzeichen haben, jedoch in deutlich geringerem Umfang als die in diesem Papier untersuchten Beschäftigungsinitiativen. Eine umfassende Übersicht über kommunale Aktivitäten im Bereich „Hilfe zur Arbeit" liefern Böckmann-Schewe/Röhrig (1997). 
unterschiedlich hohen Anteil an Personen zu vermitteln, die gleichzeitig mehrere Vermittlungshemmnisse aufweisen.

Die Zielgruppen und Arbeitsweisen der untersuchten Beschäftigungsinitiativen sind nachfolgend aufgelistet:

1. GOAB

Alle arbeitsfähigen ${ }^{13}$ Sozialhilfeempfänger, die seit mindestens drei Monaten Sozialhilfe beziehen, werden zu einer Erstberatung der GOAB eingeladen; die Auswahl der Teilnehmer wird vom Sozialamt durchgeführt (vgl. BöckmannSchewe/Röhrig 1997, 124). Darüber hinaus sind seit Mitte 1997 alle Neuantragsteller verpflichtet, an einem Erstberatungsgespräch teilzunehmen (passives Marketing). Sofern für eine bestimmte Maßnahme oder einen bestimmten Arbeitsplatz eine Arbeitskraft gesucht wird, sucht die GOAB gezielt nach Arbeitskräften (aktives Marketing). Neben den herkömmlichen Mitteln (Anzeige, Aushang, etc.) haben sich in der Arbeit mit Langzeitarbeitslosen Informationsveranstaltungen als nützlich erwiesen, welche dazu beitragen, Hemmungen und Vorbehalte abzubauen, und somit die Akzeptanz der Maßnahme erhöhen (vgl. Schulze-Böing/Simon, 1998, 13f). Darüber hinaus überweist auch das Arbeitsamt Langzeitarbeitslose an die GOAB. Insgesamt führt die GOAB derzeit jährlich etwa 1.400 Erstberatungsgespräche durch und nimmt etwa 170 bis 200 Personen in inr Beschäftigungsprogramm auf.

2. Fallmanagement Sozialamt Offenbach

Die Sachbearbeiter des Sozialamts Offenbach haben seit November 1996 die Möglichkeit, einzelne, ihrer Meinung nach geeignete Fälle zur besonderen Betreuung an das Referat Fallmanagement abzugeben. Dies betrifft grundsätzlich arbeitsfähige Sozialhilfebezieher mit einem oder mehreren Vermittlungshemmnissen, die durch gezielte Betreuung und Beratung zu beseitigen sind. Nach erfolgreicher Bewältigung der Vermittlungshemmnisse finden die Sozialhilfebezieher entweder selbständig Arbeit oder nehmen die Vermittlungs-

13 Als nicht arbeitsfähig werden Personen mit gesundheitlichen Beeinträchtigungen - körperlich und psychisch -, mit Suchtproblemen und Frauen in Schwangerschaft oder mit betreuungspflichtigen Kindern eingestuft. Zur Abgrenzung s. 6.1. 
dienste der GOAB in Anspruch. Von November 1996 bis November 1997 überwiesen die Sachbearbeiter des Sozialamts sukzessiv 153 Fälle an das Referat Fallmanagement. Die Zielgruppe umfaßt demzufolge ausschließlich Sozialhilfeempfänger.

3. SiT - Selbsthilfe im Taunus

Eine sogenannte Clearing-Stelle fungiert als Anlaufstelle ausschließlich für Sozialhilfeempfänger des Main-Taunus-Kreises. Im gleichen Gebäude wie das Sozialamt angesiedelt, führen die Vermittlungskräfte vornehmlich mit Erstantragstellern Beratungsgespräche durch, um die Verweilzeiten in der Sozialhilfe so kurz wie möglich zu halten. Die Mitarbeiter der SiT-Betriebe und die Teilnehmer an den SiT-Projekten bewerben sich entweder selbständig oder werden über die Clearing-Stelle empfohlen. Im ersten Halbjahr 1998 führte die Clearing-Stelle 500 Erstberatungsgespräche durch. Darüber hinaus fanden 1997322 Personen in den SiT-internen Betrieben und Projekten Beschäftigung mit dem mittelfristigen Ziel der Vermittlung in den Ersten Arbeitsmarkt. Die Zielgruppe besteht aus Langzeitarbeitslosen und arbeitsfähigen Sozialhilfebeziehern.

\section{START Zeitarbeit NRW}

Die Teilnehmer von START rekrutieren sich vornehmlich über das Arbeitsamt, zu einem wachsenden Anteil über informelle Kanäle und nur in geringem Umfang über das Sozialamt. So waren 1997 unter 2.166 eingestellten Leiharbeitskräften nur 114 zuvor sozialhilfeberechtigt. Die spezielle Zielgruppe besteht aus Langzeitarbeitslosen, Behinderten, über 50-jährigen, rehabilitierten Suchtkranken, Berufsrückkehrern, Ausländern und Geringqualifizierten. Der Anteil der Zielgruppe bei den Neueinstellungen sinkt jedoch seit 1995 stetig (1995: 63,2 \%, 1996: 49,1\%, 1997: 44,7\%). Ein Grund hierfür liegt im Auslaufen aller zur Verfügung stehenden Fördermittel Ende 1997, wodurch eine Konzentration auf leichter vermittelbare Personen betriebswirtschaftlich notwendig wurde (vgl. Vanselow/Weinkopf 1998, 14ff). 


\section{Projekt 1.000 Ressourcen}

Die Teilnehmer des achtmonatigen Projektprogramms werden anläßlich einer Informationsveranstaltung gewonnen. Zu dieser gelangen die Sozialhilfeempfänger entweder nach Aufforderung durch ihr Sozialamt oder freiwillig aufgrund der vom Projektteam durchgeführten Werbung (bspw. Plakatwerbung im ÖPNV). Innerhalb der anderthalbstündigen Informationsveranstaltung stellen ein REGE-Berater und ein Trainer Konzept und Ablauf des nächsten Kurses vor. Interessierte Sozialhilfeempfänger schließen im Rahmen eines Einzelgesprächs im Anschluß an die Veranstaltung eine schriftliche Vereinbarung mit den Projektmitarbeitern ab, die alle Leistungen und Erwartungen an die Teilnehmenden enthält. Wer an dem Projekt teilnehmen möchte, darf keine Bezüge vom Arbeitsamt erhalten, muß eine Arbeitserlaubnis in Deutschland besitzen, gesundheitlich nicht beeinträchtigt sein sowie über ausreichende Deutschkenntnisse verfügen. Die insgesamt 61 Teilnehmer der im Jahr 1997 durchgeführten drei Kurse - unter ihnen neun Abbrüche innerhalb der ersten acht Wochen - stellten einen repräsentativen Querschnitt aller arbeitsfähigen Sozialhilfeempfänger Bielefelds dar.

\section{Internationaler Bund Darmstadt}

Aufgrund der zahlreichen Projekte ist eine eindeutige Abgrenzung der Zielgruppe schwer möglich. Im Auftrag der Stadt führt der Internationale Bund mit allen Neuantragstellern für Sozialhilfe Erstberatungsgespräche durch (793 Teilnehmer, davon 59 in den Ersten Arbeitsmarkt vermittelt $=7,4 \%)^{14}$. Desweiteren dienen Orientierungslehrgänge (152 Teilnehmer, davon 26 vermittelt $=17,1 \%)$ und Sprachkurse (28 Teilnehmer) als vermittlungsvorbereitende Maßnahmen.

\subsubsection{Untersuchungskriterien}

Um die Effektivität und Effizienz von Beschäftigungsinitiativen vergleichen zu können, bedarf es sowohl einer Betrachtung ihrer Strukturdaten als auch ihres

14 Die Daten beziehen sich auf den Zeitraum Januar bis August 1998, Internationaler Bund Darmstadt. 
Erfolgs hinsichtlich ihrer spezifischen Zielsetzung. Zu den Strukturdaten zählen Trägerschaft, Rechtsform, Größe (Anzahl der Maßnahmenteilnehmer), Aufgabenfelder, Personal, Angebote sowie Finanzierungsquellen.

Der Erfolg von Beschäftigungsinitiativen läßt sich neben der Vermittlungsquote an der Entlastung des Sozialhaushaltes sowie der Verringerung der psychosozialen, medizinischen und sozialpolitischen Kosten der Langzeitarbeitslosigkeit messen. Die Arbeit von Beschäftigungsinitiativen besitzt somit neben einer beschäftigungspolitischen auch eine finanz- und sozialpolitische Dimension. Die Bewertung der Vermittlungstätigkeit muß allerdings vor dem Hintergrund der regionalen Wirtschafts- und Arbeitsmarktlage und den institutionellen Rahmenbedingungen (s. Kapitel 2) erfolgen. In Anlehnung an Wilk (1997b, 105ff) wurden der Analyse die folgenden Untersuchtungskriterien zugrundegelegt:

\section{Effektivität und Zielgruppenadäquanz}

Welcher Art und mit welchen Wirkungen finden Selektionsprozesse statt? Werden Langzeitarbeitslose und arbeitsfähige Sozialhilfeempfänger effektiv eingebunden, welche Gruppen werden diskriminiert?

\section{Integrationsleistungen}

Es empfiehlt sich hierbei eine Aufgliederung in drei Phasen, wobei die Bewältigung einer jeden dieser Phasen als Erfolg zu bewerten ist:

Phase I: Projektintegration (Übergang aus der Arbeitslosigkeit in das Projekt)

Phase II: Qualifizierungsphase (Voraussetzungen zur Vermittlung schaffen)

Phase III: Arbeitsmarktintegration (Übergang aus dem Beschäftigungsprojekt in ein Arbeitsverhältnis)

Die reine Vermittlungsquote, d.h. das Verhältnis der Anzahl der in Arbeit vermittelten Teilnehmer zu der Anzahl der Abgänger insgesamt, birgt als Erfolgskriterium Risiken. „Die Quote der in Arbeit vermittelten ehemaligen Maßnahmeteilnehmer gibt nur in sehr eingeschränktem Maße Auskunft über tatsächliche Eingliederungserfolge, denn die Vermittlung in reguläre Be- 
schäftigung ist nur das Ende eines mitunter langwierigen beruflichen und sozialen Eingliederungsprozesses“ (Wilk 1997b, 106f).

\section{Beschäftigungswirkungen}

Wie sieht die Situation der ausgeschiedenen Teilnehmer sechs bzw. zwölf Monate nach der Vermittlung aus? Erst wenn die Vermittlungen dauerhaft sind, kann auch von einer Beschäftigungswirkung gesprochen werden, vorausgesetzt es fanden keine Verdrängungseffekte statt.

\section{Kosten}

Das Verhältnis der Entlastungswirkung zu den quantifizierbaren Brutto- und Nettokosten ist für die Kommunen eines der wichtigsten Erfolgskriterien. Bei zu kurzfristiger Betrachtungsweise besteht die Gefahr des sogenannten fiskalischen 'Drehtüreneffektes': Sozialhilfeempfänger erhalten für die Dauer eines Jahres sozialversicherungspflichtige Beschäftigung auf dem kommunalen Zweiten Arbeitsmarkt, wodurch sie Ansprüche auf Leistungen der Arbeitsverwaltung erhalten, bis diese nicht mehr ausreichen, um den Lebensunterhalt zu finanzieren oder die Bezugsdauer abgelaufen ist und die Arbeitslosen wieder ergänzend oder voll Sozialhilfe beziehen.

\subsubsection{Zwischenfazit}

Die in dieser Studie untersuchten Beschäftigungsinitiativen erreichen hinsichtlich der aufgestellten Erfolgskriterien unterschiedliche Ergebnisse, hervorgerufen durch uneinheitliche regionale Voraussetzungen sowie leicht differierende Zielvorgaben. Die Effektivität ihrer Arbeit hängt nicht zuletzt von der Dauer ihrer Tätigkeit ab. So bedarf beispielsweise eine funktionierende Stellenakquise, die Teil der Vermittlungstätigkeiten in den untersuchten Beschäftigungsinitiativen ist, einer gewissen Anlaufzeit, um einerseits einen umfangreichen Stellenund Bewerberpool aufzubauen und andererseits die Vorbehalte auf Arbeitnehmer- sowie Arbeitgeberseite abzubauen. Der gemeinsame Ansatz - ein Gesamtkonzept aus mehreren integrierten Maßnahmen, eingebettet in ein eng zusammenarbeitendes Netz der zuständigen Stellen - kann in bezug auf die Verringerung von Langzeitarbeitslosigkeit als problemadäquat bezeichnet wer- 
den, weist jedoch einige spezifische Probleme und Schwächen auf, die im folgenden Abschnitt skizziert werden.

\subsubsection{Probleme von Beschäftigungsinitiativen}

Die Arbeit von Beschäftigungsinitiativen wird von einer Reihe von Problemfeldern erschwert, die sich innerhalb der in den beiden folgenden Kapiteln untersuchten Problemgruppen einordnen lassen.

\subsubsection{Mangel an Ressourcen}

Im Hinblick auf ihre Finanzierungsquellen lassen sich Beschäftigungsinitiativen in eine kleinere Gruppe der sich selbst tragenden, d.h. ohne Zuschüsse wirtschaftenden, und in eine größere Gruppe der durch Eigenerwerb und Zuschüsse aus den verschiedensten Quellen finanzierten Beschäftigungsinitiativen aufteilen. Der Anteil der Zuschüsse wird aufgrund der angespannten Haushaltslage vieler öffentlicher Träger in Zukunft vermutlich zurückgehen, was die Gruppe der bezuschußten Beschäftigungsinitiativen zu marktnäherem Verhalten zwingt.

\section{"Creaming-poor"}

Sich selbst tragende bzw. marktnahe Beschäftigungsinitiativen zeichnen sich durch einen relativ geringen Anteil an Sozialhilfeempfängern und Langzeitarbeitslosen aus (START Zeitarbeit NRW, s. Anhang). Sie stehen deswegen im 'Verdacht', ihre Vermittlungstätigkeit auf die leicht zu vermittelnden Arbeitssuchenden zu konzentrieren, um auf diese Weise beständig den 'Rahm abzuschöpfen'. "Bei der Rekrutierung von Personal werden leistungsstärkere Arbeitsanbieter den tatsächlich oder vermeintlich leistungsschwächeren Erwerbslosen vorgezogen (creaming poor)" (Wilk 1997b, 117). Dieser Effekt, meist als Problem tituliert, hat jedoch auch positive Folgen. Konzentriert sich die Vermittlungstätigkeit zunächst auf die leichter eingliederbaren Arbeitssuchenden, so können mit vergleichsweise geringem Mitteleinsatz hohe Vermittlungszahlen erreicht werden. 
Für die erst seit kurzer Zeit Sozialhilfe beziehenden Arbeitslosen besitzt diese Vorgehensweise einen präventiven Charakter, da die Dauer der Arbeitslosigkeit eng mit dem Zuwachs an vermittlungshemmenden Merkmalen korreliert ist (vgl.: G.I.B. - Gesellschaft für innovative Beschäftigungsförderung 1998, 13, und Kap. 2.1, ad. 1). Gute Erfahrungen machen verschiedene Beschäftigungsinitiativen mit für Neuantragsteller verpflichtenden Erstberatungsgesprächen, wodurch viele Arbeitssuchende eine Stelle finden, ohne weitere Maßnahmen durchlaufen zu müssen. Sie erhalten somit frühzeitig die Chance, aus der $\mathrm{Ne}$ gativspirale von Nichtbeschäftigung und Humankapitalverlust auszubrechen.

Vermittelt die Beschäftigungsinitiative viele Arbeitskräfte, steigen ihre Einnahmen aus der Vermittlungstätigkeit. Mit Hilfe dieser Mittel können qualifizierende Maßnahmen finanziert werden, um auch schwerer Vermittelbare in das Programm aufzunehmen. Ein solches gemeinnütziges Verhalten muß jedoch Aufnahme in die Satzung der Beschäftigungsinitiative finden und regelmäßigen Prüfungen durch unabhängige Stellen unterzogen werden. Eine weitere Folge der Konzentration auf leicht Vermittelbare ist der mögliche Reputationsgewinn der Beschäftigungsinitiative bei den Arbeitgebern, vorausgesetzt die vermittelten Kräfte arbeiten zuverlässig. Im Zuge eines Reputationsgewinns ist es aufgrund des aufgebauten Vertrauens möglich, vermehrt Arbeitsplätze für schwer Vermittelbare zu schaffen.

Gleichwohl besteht die Möglichkeit, daß Beschäftigungsinitiativen schwer Vermittelbare nie in ihre Vermittlungstätigkeit aufnehmen. Aufgrund der hohen Fluktuation innerhalb der Sozialhilfe wird die Zahl der leicht Vermittelbaren wahrscheinlich selbst bei erfolgreicher Arbeit der Initiative kein derart niedriges Niveau erreichen, daß sie gezwungen ist, sich um die Gruppe der schwer Vermittelbaren zu kümmern, will sie nicht ihre Arbeit einstellen. Besonders schwer vermittelbare Personen werden in der Regel an das Sozialamt zurückverwiesen, so daß das Risiko besteht, daß manche Personen dauerhaft von der Vermittlung ausgeschlossen werden. An dieser Stelle setzt der vornehmlich sozialpolitisch geprägte Vorwurf des "creaming-poor" an. Findet dieser Vorwurf bei den zuständigen Stellen Gehör, besteht bei zu starker Konzentration auf die Gruppe der schwer Vermittelbaren die Gefahr des Reputationsver- 
lustes der Beschäftigungsinitiativen. Zudem wird dabei übersehen, daß eine produktivitätsorientierte Vermittlung gesamtwirtschaftlich effizient ist.

\section{Mangelnde Reputation von Beschäftigungsinitiativen}

Der Erfolg der Vermittlungstätigkeit der untersuchten Beschäftigungsinitiativen ist unter anderem von einer umfangreichen und funktionierenden Stellenakquise abhängig. Die Arbeit des Vermittlers besteht zu einem Großteil im Aufspüren von freien Stellen auf dem regionalen Arbeitsmarkt. Will er Langzeitarbeitslose und arbeitsfähige Sozialhilfeempfänger in diese freien Stellen vermitteln, muß er in den meisten Fällen zunächst die Vorbehalte der Unternehmer gegenüber dieser Klientel ausräumen. Es ist offensichtlich, daß die Schwierigkeit seiner Aufgabe direkt von der Arbeitsqualität der bisher vermittelten Personen abhängt. "Die Betriebe als Kunden, die zwischen verschiedenen Alternativen wählen können, müssen von der Qualität der Dienstleistung überzeugt werden" (Vanselow/Weinkopf 1998, 52f). Gelingt dies nicht, laufen alle sonstigen Maßnahmen zur Vermittlung letztlich ins Leere (vgl. Vanselow/Weinkopf 1998, 52f und 63 sowie Kehler 1998, 15).

Dennoch ist nicht nur aus sozialpolitischen Gründen ein gewisser Anteil an schwer Vermittelbaren tragbar. Oftmals zeigen die auf den ersten Blick hoffnungslosen Fälle nach intensiven Beratungsgesprächen und eventuellen Probebeschäftigungen unbekannte Qualitäten verbunden mit einer besonders hohen Motivation. Solche Erfahrungen verzeichnet u.a. das in der Eingliederung schwer Vermittelbarer relativ erfolgreiche Projekt '1.000 Ressourcen', dessen Leitbild neben der Vermittlung in den Ersten Arbeitsmarkt "die Steigerung der Selbstvermittlungskompetenz, des Selbstvertrauens, der Kontaktaufnahme, Kommunikationsfähigkeit und Beziehungsgestaltung" (Adil 1998, 159) ist. Die Kehrseite der Medaille ist der hierfür notwendige hohe Personalbedarf mit einem Vermittler sowie sechs qualifizierten Trainern pro sechzig Arbeitssuchenden pro Jahr. 


\subsubsection{Geringe Vermittlungszahlen}

Die nachfolgenden Aussagen beziehen sich vornehmlich auf Kommunen, die ein integriertes Gesamtkonzept zur (Wieder-)Eingliederung von Langzeitarbeitslosen und Sozialhilfeempfängern in den Ersten Arbeitsmarkt verfolgen (inkl. Erstberatungsgespräche). Die absoluten Vermittlungen ${ }^{15}$ aller Beschäftigungsinitiativen sind in Relation zu der Anzahl der Langzeitarbeitslosen und arbeitsfähigen Sozialhilfebezieher gering. Die Gründe für diesen Umstand und Möglichkeiten zur Verbesserung der Lage liegen dabei sowohl auf der Seite des Arbeitsangebots als auch auf der Seite der Arbeitsnachfrage.

\section{Arbeitsangebot}

Eine Vermittlung durch die Beschäftigungsinitiative ist entweder direkt nach einem Erstberatungsgespräch oder erst nach Abschluß einer oder mehrerer Maßnahmen möglich. Im Hinblick auf die absoluten Vermittlungszahlen ist der Bereich der Direktvermittlungen größer als derjenige nach Maßnahmenende. Bei den Vermittlungsquoten hingegen dreht sich das Verhältnis um: Während auf ersterem Weg etwa 10 bis $20 \%$ aller Teilnehmer direkt in Arbeit vermittelt werden können, schaffen - je nach Ausgestaltung und Qualität des Gesamtkonzepts - 15 bis $40 \%$ aller Maßnahmenteilnehmer den Sprung in den Ersten Arbeitsmarkt. Diese Spanne der Quoten hängt u.a. von konjunkturellen und regionalen Einflüssen ab (vgl. Schomburg, 1994, 157).

Um die absoluten Vermittlungszahlen bei gegebener Arbeitsnachfrage zu erhöhen, müßten entweder

- mehr Arbeitssuchende an Erstberatungsgesprächen bzw. Maßnahmen teilnehmen oder

- eine höhere Quote der Absolventen eine Arbeit finden.

Den meisten Erfolg verspricht hierbei die Ausweitung der Erstberatungsgespräche, womit zusätzlich die o.a. positiven Wirkungen für Neuantragsteller verbunden wären (vgl. Stadt Offenbach am Main 1998b, $4 \mathrm{f}$ und Anhang, 6 : Projektintegration). Eine zahlenmäßige Erhöhung der Maßnahmenteilnehmer

\footnotetext{
${ }^{15}$ Zu den Zahlenangaben siehe den Anhang.
} 
ist für die untersuchten Beschäftigungsinitiativen aufgrund der finanziellen und damit personellen Restriktionen nur sehr begrenzt möglich. Eine Ausnahme bildet hierbei das Projekt '1.000 Ressourcen', welches derzeit weitere Trainer ausbildet, um einen größeren Teilnehmerkreis aufnehmen zu können. Jedoch begrenzen die komplexe, neunmonatige Trainer-Ausbildung und das angestrebte Trainer-Teilnehmer-Verhältnis von 1 zu 10 die Geschwindigkeit einer Ausdehnung derart, daß auf diese Weise der Projektcharakter noch einige Jahre erhalten bleiben wird. Die bisherigen Untersuchungen der einzelnen Beschäftigungsinitiativen zeigen, daß Verbesserungsmöglichkeiten bei den Instrumenten auf der Angebotsseite in ihrer Wirkung auf die Vermittlungswahrscheinlichkeit insgesamt begrenzt sind und somit nachfragewirksame Instrumente wie Lohnsubventionen unabdingbar sind. Jüngere Erfahrungen u.a. auch in anderen Ländern zeigen zudem, daß ein gewisser vornehmlich monetärer Druck auf Leistungsbezieher verbunden mit einer Neubewertung von Eigenverantwortung vermittlungsförderlich ist ${ }^{16}$.

\section{Arbeitsnachfrage}

Der Arbeitsbereich von Beschäftigungsinitiativen auf der Seite der Arbeitsnachfrage liegt im Aufspüren eines unbefriedigten Arbeitskräftebedarfs, welcher ohne die Vermittlungstätigkeit der Initiativen nicht gedeckt würde. Unbefriedigter Arbeitskräftebedarf existiert beispielsweise bei offenen Stellen, die trotz Bemühung der Unternehmen nicht besetzt werden konnten. Sogenannte 'versteckte' Arbeitsplätze kennzeichnen einen unbefriedigten Arbeitskräftebedarf, welchem kein Suchprozeß vorausging. Dieser tritt vor allem bei kleinen Unternehmen auf, die es sich aus zeitlichen, personellen oder auch finanziellen Gründen nicht leisten können, eine Stelle auszuschreiben und eventuelle Bewerber zu testen. Gerade für diese kleinen Unternehmen wird der Begriff des Dienstleistungsunternehmens deutlich, welches die Beschäftigungsinitiative für

16 Das sog. „New Deal"-Programm der britischen Regierung zur Verringerung der Jugendarbeitslosigkeit folgt dem Prinzip „fordern und fördern". In Dänemark wurde Mitte der 90er Jahre im Zuge von Arbeitsmarktreformmaßnahmen die Balance zwischen Transferleistungen und Druck zur Arbeitsaufnahme insbesonders durch schärfere Anspruchskriterien beim Leistungsbezug verschoben (vgl. Emmerich 1998). 
Arbeitssuchende, Unternehmer und Kommune darstellt (vgl. SchulzeBöing/Simon 1998, 5; Vanselow/Weinkopf 1998, 52f; SiT-Selbsthilfe im Taunus e.V. 1997, 1 und Adil 1998, 159).

Gleichwohl ist die Zahl dieser Arbeitsplätze begrenzt: "Nach wie vor ist der regionale Arbeitsmarkt gekennzeichnet von einem erheblichem Defizit an Einfacharbeitsplätzen, die Beschäftigung eines Großteils der arbeitsfähigen und arbeitswilligen Sozialhilfebezieherinnen und Bezieher bieten können" (Stadt Darmstadt 1998, 8). Darüber hinaus wird bis zum Jahr 2010 der Wegfall von weiteren rund drei Millionen Einfacharbeitsplätzen vor allem im produzierenden Gewerbe prognostiziert (vgl. Bundesinstitut für Berufsbildung 1995, 24).

Der einzige Ausweg aus diesem Dilemma ist die signifikante und dauerhafte Erhöhung von Einfacharbeitsplätzen insbesondere im Dienstleistungsbereich auf dem Ersten Arbeitsmarkt.

\subsection{Schlußfolgerungen}

Betrachtet man die bereits existierenden arbeitsmarktpolitischen Maßnahmen und Instrumente, so kann man nicht umhin, zweierlei zu konstatieren:

Erstens: Es gibt bereits eine Menge verschiedenster Instrumente und Zweitens: Für die Arbeitsmarktpolitik im speziellen gilt die gleiche Kritik wie für die Sozial- und Steuerpolitik im allgemeinen.

Die herrschende Arbeitsmarktpolitik mit ihrem Ziel der Einzelfallgerechtigkeit hat zu einer unüberschaubaren und unkoordinierten Flut von Einzelmaßnahmen unterschiedlichster Maßnahmenträger geführt. Als Beispiel sei hier nur auf den beschäftigungspolitischen Aktionsplan der Bundesregierung (BMWi 1998) verwiesen. Hierin werden Maßnahmen von Bund, Ländern und Kommunen aufgelistet, die auf unterschiedlichen Gesetzesvorgaben beruhen, gleichzeitig aber auf gleiche Erwerbspersonengruppen abzielen, mithin selbst Probleme durch dieses unkoordinierte Vorgehen produzieren. Hinzu kommt, daß durch diese Intransparenz keine Signalwirkung von einzelnen Maßnahmen ausgehen kann, da sie oft nicht wahrgenommen werden, so daß sie an den wirklich Bedürftigen häufig vorbei geht. So wurden bisher die Möglichkeiten zu Lohnko- 
stenzuschüssen von den Arbeitgebern aus Informationsmangel nicht ausgeschöpft (vgl. Hackenberg/Wagner 1996).

Im Hinblick auf die hier im Mittelpunkt stehende Gruppe der langzeitarbeitslosen Sozialhilfe- und Arbeitslosenhilfeempfänger kommt schließlich ein weiteres Problem der Instrumentenvielfalt und -intransparenz zum Tragen: Denn in deren Folge kommt es zu Verteilungskämpfen sowohl zwischen den verschiedenen Maßnahmenträgern - also insbesondere Bund, Kommunen und Arbeitslosenversicherung - als auch zwischen alten und neuen Maßnahmen bzw. deren institutionellen Pendants. Dieser Mechanismus ist gesamtwirtschaftlich und insbesondere beschäftigungspolitisch ineffizient, da das Beschäftigungsziel hinter dem jeweiligen Streben nach Haushaltskonsolidierung zurücktritt. Schließlich sind die verschiedenen Träger und hier insbesondere die Arbeitsämter mit der Betreuung von Langzeitarbeitslosen häufig überfordert.

Fort- und Weiterbildungsmaßnahmen, vor allem für gering qualifizierte Personen, die den Schwerpunkt klassischer aktiver Arbeitsmarktpolitik bilden, stossen zudem an diverse, bereits diskutierte Grenzen, so daß die Schaffung von Arbeitsplätzen gerade für diese wenig wettbewerbsfähige Arbeitnehmergruppe im Mittelpunkt arbeitsmarkt- und sozialpolitischer Maßnahmen stehen sollte. Denn die durchaus richtige Politik der Humankapitalbildung und -förderung führt lediglich zu Arbeitslosigkeit von besser ausgebildeten Personen, wenn für sie keine Arbeitsplätze zur Verfügung stehen. Diese Gefahr besteht, wenn am Markt vorbei ausgebildet wird oder es sich nur um reine Beschäftigungsmaßnahmen handelt. Insbesondere muß die Entstehung geschützter, dem Wettbewerb entzogener Arbeitsplätze, die damit wiederum Arbeitsplätze im Wettbewerbssektor gefährden könnten, vermieden werden (Verdrängungseffekte). Schließlich müssen allzu bekannte Drehtüreneffekte bzw. Maßnahmenkarrieren, welche die davon betroffenen Personen zusätżlich demotivieren, abgebaut werden. Zusammenfassend gelingt es der bisherigen Arbeitsmarktpolitik nur sehr eingeschränkt, die Ziele der Sozial-, Arbeitsmarkt- und Wirtschaftsintegration zu realisieren. Das Konzept des Zweiten Arbeitsmarktes scheitert letztlich zwangsläufig am - hier nicht lösbaren - Widerspruch von der Notwendigkeit marktfähige Qualifikationen zu vermitteln und dem Zusätzlichkeitskriterium. 
Die Analyse der bisherigen Arbeitsmarktpolitik läßt deshalb einen neuen Weg zur Integration Langzeitarbeitsloser als notwendig und sinnvoll erscheinen. Dabei wird eine Integration der Zielgruppe in den Ersten Arbeitsmarkt - ohne den bisherigen Umweg über den Zweiten Arbeitsmarkt - durch die Kombination zweier Instrumente zu erreichen versucht: Lohnsubventionen sollen zum einen die allgemeine Konkurrenzfähigkeit des spezifischen Angebots und die Arbeitsnachfrage danach erhöhen. Zum anderen soll die individuelle und nachhaltige Wettbewerbsfähigkeit der Zielgruppenmitglieder durch eine Beratungs-, Betreuungs- und Vermittlungsagentur gefördert werden. Letzteres ist insbesondere deshalb angezeigt, da beim Übergang von Sozialhilfebeziehenden in den Arbeitsmarkt zwei Probleme auftreten: Erstens kann man eine Orientierungslosigkeit beobachten; viele Hilfeempfänger kennen ihre eigenen Stärken und Schwächen nicht und können dementsprechend gar nicht beurteilen, welche beruflichen Perspektiven sie haben. Zweitens ist der Wunsch, nach langer Arbeitslosigkeit wieder in das Berufsleben zurückzukehren, ebenso gering wie die Bereitschaft, Hilfen anzunehmen (vgl. Wilk 1997a, 286).

Das alleinige Ziel dieses Vorschlags ist die Integration Langzeitarbeitsloser in den Ersten Arbeitsmarkt. Damit steht diese Maßnahme im Gegensatz zur Hilfe zur Arbeit im Rahmen der Sozialhilfe, wo neben der Arbeitsmarktintegration auch eine Sozial- und Wirtschaftsintegration angestrebt wird (vgl. Wilk 1997a, 285). Wie aber weiter oben gezeigt werden konnte, wird das Instrument der Hilfe zur Arbeit dadurch überfrachtet, und die interne Effizienz - also die Effizienz einer Maßnahme hinsichtlich ihres expliziten Zieles - wird nicht oder nur unzureichend erreicht, was infolge der Zieldimensionen auch nicht verwundern kann. Vor dem Hintergrund, daß die Beschäftigung im Rahmen einer Erwerbsarbeit selbst eine hohe soziale und ökonomische Integrationswirkung nach sich zieht, erscheint eine Konzentration auf die Arbeitsmarktintegration als notwendige und sinnvolle Zielformulierung. In den beiden folgenden Kapiteln werden zunächst die Lohnsubventionen und anschließend die Agenturüberlegungen konkretisiert. 
Martin Setzer, Roland Klopfleisch and Werner Sesselmeier - 978-3-631-75277-7

Downloaded from PubFactory at 01/11/2019 06:22:32AM

via free access 


\section{Lohnsubventionen zur Integration Langzeitarbeitsloser}

Die Subventionierung geringqualifizierter Tätigkeiten kann zum einen sowohl auf der Arbeitsangebotsseite als auch auf der Nachfrageseite anknüpfen und zum anderen kann sie in Form von direkten Zuschüssen oder in Verrechnung mit der individuellen Steuerschuld in Anlehnung an eine negative Einkommensteuer erfolgen. Die theoretische Vorteilhaftigkeit der verschiedenen Möglichkeiten ist noch nicht abschließend geklärt. Für einen regional, zahlenmäßig und zeitlich begrenzten Modellversuch, der vor dem Hintergrund der herrschenden Arbeitslosigkeit und den Erfahrungen im Ausland mit derartigen Instrumenten nicht nur als sinnvoll, sondern auch als äußerst dringlich zu bezeichnen ist, bietet sich - wie zu zeigen ist - eine direkte Subventionierung der Lohnkosten an.

Im Rahmen dieses Kapitels sollen Lohnsubventionen zunächst allgemein erläutert werden, dann hinsichtlich des formulierten Auftrags konkretisiert und diskutiert werden.

\subsection{Definition und Klassifikation von Lohnsubventionen}

Lohnsubventionen sind hinsichtlich des Ziels, spezifische Arbeitnehmergruppen zu fördern, als zielgruppengenaues und anreizkonformes Instrument einzustufen (Vierling 1997, 459). Dabei wird hier der Begriff der Lohnsubvention nicht in der üblichen Weise abgegrenzt. So definiert beispielsweise Andel $(1970,5)$ den Subventionsbegriff sehr eng als diejenigen Geldzahlungen, die ohne marktliche Gegenleistungen von den öffentlichen Händen an diejenigen Unternehmen fließen, die bestimmte Voraussetzungen erfüllen. Für die hier zu verfolgende Argumentation ist zunächst einmal die von Albeck $(1982,9)$ gegebene Definition brauchbarer: "Lohnsubventionen (LS) sind geldwerte Vorteile, die der Staat Arbeitgebern für die Beschäftigung von Arbeitnehmern gewährt, sei es als Geldzahlungen, als Befreiung von Abgabepflichten oder als ähnliche Begünstigungen. Ihr wesentlicher Zweck besteht darin, den Produktionsfaktor Arbeit zu verbilligen und da- 
durch ein höheres Beschäftigungsniveau und/oder eine andere Beschäftigungsstruktur zu erreichen als ohne LS zustande gekommen wäre."

Von dieser Definition ausgehend geht es nicht darum, einzelne arbeitsmarktpolitische Maßnahmen unter dem Begriff der Lohnsubvention zu subsumieren und dann zu beurteilen. Vielmehr geht es um die Analyse direkter Geldleistungen an die Unternehmen oder - in Erweiterung der Albeck'schen Definition - an Arbeitnehmer, um deren Lohn(kosten) zu senken.

Lohnsubventionen können zunächst bezüglich der Bezugsdauer, des begünstigten Personenkreises und der Bemessungsgrundlage klassifiziert werden (vgl. hierzu Bohlen 1993, 7ff). ${ }^{17}$

Als erstes lassen sie sich hinsichtlich ihrer zeitlichen Gewährung in temporäre und permanente Lohnsubventionen unterscheiden. Permanente Lohnsubventionen werden über einen längeren Zeitraum bewilligt, sind also zunächst zeitlich nicht befristet, während temporäre Lohnsubventionen einen nur vorübergehenden Charakter aufweisen. Aufgrund dieses Unterschiedes gilt häufig, daß die effektiven Wirkungen temporärer Lohnsubventionen auf den Arbeitsmarkt nur kurzfristiger Natur sind. Bei Gewährung einer zeitlich befristeten Lohnsubvention werden Unternehmen in erster Linie nur bestimmte, bereits getroffene Entscheidungen über die Neueinstellung von Arbeitskräften zeitlich vorziehen, um in den Genuß der Lohnsubvention zu gelangen. Im Gegensatz zu permanenten Lohnsubventionen führen temporäre Lohnsubventionen in der Regel nicht zu einer nachhaltigen Mehrnachfrage von Unternehmen auf dem Arbeitsmarkt, sofern man unterstellt, daß die Unternehmen in ihrer Personal- und Investitionsplanung einen langfristigen Zeithorizont besitzen. Andererseits müssen bei der Beurteilung von Lohnsubventionen auch die dem Staat als Subventionsträger entstehenden Kosten berücksichtigt werden. Diese sind bei der

17 Möglich wäre auch eine Unterscheidung zwischen Subventionen, die die Zahl der Erwerbstätigen erhöhen können im Sinne der hier zu diskutierenden Lohnsubventionen, und Subventionen, die zum Abbau der Arbeitslosigkeit dadurch beitragen, daß sie das Erwerbspersonenpotential verkleinern. Zur zweiten Gruppe gehören beispielsweise Frühverrentungen und Vorruhestandsregelungen. Genauso stellen ABM im ökonomischen Sinne keine Subvention dar. In dieser Studie werden nur Subventionen der ersten Gruppe berücksichtigt, siehe zur Analyse der zweiten Subventionskategorie Schellhaaß/Kelp 1995. 
Gewährung permanenter Lohnsubventionen weitaus höher als bei temporären. Daher können auch zeitlich befristete Subventionen unter gewissen Umständen wirtschaftspolitisch durchaus sinnvoll sein, etwa um zu verhindern, daß kurzfristige gesamtwirtschaftliche Nachfrageausfälle voll auf den Arbeitsmarkt durchschlagen.

Desweiteren kann eine Unterscheidung in allgemeine und zielgruppenorientierte Lohnsubventionen erfolgen. Im ersten Fall wird jegliche Art der Beschäftigung gefördert, d.h. sämtliche innerhalb eines vorgegebenen Zeitraums geschlossenen Arbeitsverträge werden subventioniert. Durch den zweiten Fall der zielgruppenorientierten Lohnsubventionen werden hingegen nur bestimmte Arbeitnehmer gefördert, d.h. es erfolgt eine differenzierte Vergabe von Lohnsubventionen. Mit der gezielten Förderung von ganz bestimmten Arbeitnehmergruppen wird versucht, die Chancen dieser Gruppen auf dem Arbeitsmarkt zu verbessern. Zielgruppenorientierte Lohnsubventionen werden deswegen primär für die Beschäftigung von Arbeitnehmern gewährt, die auf dem Arbeitsmarkt nur schwer vermittelbar sind und häufig von Langzeitarbeitslosigkeit betroffen sind. Die gezielte Förderung bestimmter Personenkreise ruft allerdings auch Substitutionseffekte bei der Arbeitsnachfrage hervor, denn teilweise werden nicht-subventionierte von subventionierten Arbeitnehmern verdrängt. Das Ausmaß dieser Verdrängung ist in erster Linie eine Funktion der Substituierbarkeit von Arbeitskräften untereinander im Produktionsprozeß und der zeitlichen Dauer der Subventionierung. Bei allgemeinen Lohnsubventionen ist ein derartiger Austausch von Arbeitskräften nicht zu erwarten, da sämtliche Arbeitnehmer subventioniert werden. Ein Austausch von Arbeitskräften erscheint für einen Unternehmer unter diesen Umständen nicht lohnend. Generell gilt, daß allgemeine Lohnsubventionen zwar ökonomisch insgesamt sinnvoller, andererseits aber die teurere Variante darstellen, weswegen eine gezielte Förderung bei geringen und zudem zeitlich begrenzten Mitteln oftmals der einzig praktikable Weg ist.

Eine weitere Form der Lohnsubventionierung ist die marginale Lohnsubvention. Bei der marginalen Lohnsubvention wird ausschließlich der Netto-Beschäfti- 
gungszuwachs gefördert, weshalb im gleichen Zusammenhang oft auch der Begriff der inkrementellen Lohnsubvention verwendet wird. Die eigentliche Intention der marginalen Lohnsubvention muß so verstanden werden, daß nur der Teil der Neuarbeitsverträge eines bestimmten Zeitraumes finanziell unterstützt wird, dem nicht ein entsprechender Teil von Vertragsauflösungen im gleichen Zeitraum gegenübersteht. Durch Einführung einer marginalen Lohnsubvention wird also versucht, die Förderung auf diejenigen Arbeitsverhältnisse zu beschränken, die in der betrachteten Periode zusätzlich entstehen. Idealerweise soll nur diejenige Beschäftigung subventioniert werden, die ohne die Gewährung der marginalen Lohnsubvention nicht entstanden wäre. Eine derartige Identifizierung der zusätzlichen Beschäftigung ist allerdings in der Praxis nur bedingt möglich. Zur Quantifizierung des Netto-Beschäftigungszugangs bedient man sich häufig sogenannter Soll-Beschäftigungen. Diese erhält man, indem zunächst der durchschnittliche Beschäftigungszuwachs aller Unternehmen für die Zukunft statistisch ermittelt und anschließend dieser Wert mit den tatsächlichen Werten der Unternehmen verglichen wird. Unternehmen, deren Beschäftigungszuwachs unterhalb des durchschnittlichen Wertes liegt, erhalten in diesem Fall keine Lohnsubventionen und erleiden im Vergleich zu Unternehmen mit überdurchschnittlichen Beschäftigungszuwächsen einen Wettbewerbsnachteil. Marginale Lohnsubventionen können selbstverständlich wiederum in allgemeiner bzw. zielgruppenorientierter Form ausgestaltet werden.

Abschließend können Lohnsubventionen noch bezüglich ihrer Bemessungsgrundlage klassifiziert werden. Man unterscheidet hierbei zwischen pauschalen Lohnsubventionen und Wertsubventionen. Bei pauschalen Subventionen handelt es sich um Lohnsubventionen, die einen konstanten Betrag je Arbeitnehmer darstellen. Im Gegensatz dazu werden Wertsubventionen als ein auf den Lohn bezogener Zuschuß gezahlt und variieren demnach in ihrer Höhe mit dem Lohn eines Arbeitnehmers. Bei einem proportionalen Tarifverlauf der Wertsubvention wächst der Subventionsbetrag mit der Bemessungsgrundlage, d.h. dem Lohn. Man spricht in diesem Zusammenhang auch von einer proportionalen Lohnsubvention. Denkbar ist auch eine zeitliche Staffelung der Zuschüsse 
einer Wertsubvention. Es kann beispielsweise vereinbart werden, daß die Zuschußsätze von Jahr zu Jahr abnehmen.

Neben diesen weithin üblichen Unterscheidungen muß noch auf eine weitere Differenzierung nach dem (direkten) Empfänger hingewiesen werden, womit auch die weiter oben zitierte Definition von Lohnsubventionen ergänzt wird. In der öffentlichen wie auch wissenschaftlichen Diskussion versteht man nämlich unter dem Begriff der Lohnsubvention häufig nur die Zahlung von Zuschüssen an Unternehmer für die Einstellung von Arbeitnehmern. Lohnsubventionen an Arbeitnehmer wird im Vergleich zu Subventionen an Arbeitgeber ein weitaus geringeres Interesse zuteil. Der Grund hierfür ist wohl in erster Linie darin zu sehen, daß nach weitverbreiteter Meinung eine Beschäftigungsexpansion nur dann erzielt werden kann, wenn der Nachfrageseite auf dem Arbeitsmarkt, also den Unternehmen, finanzielle Anreize zur vermehrten Einstellung von Arbeitskräften gewährt werden. Aufgrund der eintretenden Kostenentlastung und der faktischen Verbilligung des Produktionsfaktors Arbeit werden die Arbeitgeber mehr Nachfrage nach Arbeitskräften entfalten. Mit dieser Beurteilung der Wirkung von Lohnsubventionen an Arbeitgeber wird implizit vorausgesetzt, daß der primäre Grund von Arbeitslosigkeit zu hohe Löhne sind. Von zu hohen Mindestlöhnen sind wiederum in erster Linie Arbeitnehmer mit sehr geringer Produktivität betroffen, denn unterstellt man, daß die Unternehmen Arbeitskräfte nachfragen, solange die Grenzproduktivität der Arbeit größer ist als der Reallohn, werden Problemgruppen mit geringer Produktivität kaum Beschäftigung finden. Aus dieser primären Zielsetzung von Lohnsubventionen als Arbeitgeberstimulans lassen sich eine Reihe von typischen Charakteristika dieser Subventionen ableiten.

Lohnsubventionen an Arbeitgeber sind sehr oft beschränkt auf die Einstellung von Arbeitnehmern, die einer Problemgruppe des Arbeitsmarktes angehören, d.h. überdurchschnittlich häufig von Arbeitslosigkeit betroffen sind. Es handelt sich demzufolge i.d.R. um zielgruppenorientierte Lohnsubventionen. Um nur den Beschäftigungszuwachs zu fördern, werden Lohnsubventionen an Arbeitgeber häufig in marginaler Form konzipiert. Zudem sind sie vorwiegend temporär, weil davon ausgegangen wird, daß subventionierte Arbeiter nach einer 
bestimmten Zeit in dem Unternehmen Qualifikationen erwerben, die ihren Wert für das Unternehmen derart steigern, daß sie den Marktlohn erhalten und auf eine weitere Bezuschussung verzichtet werden kann.

Im Gegensatz zu Lohnsubventionen an Arbeitgeber setzen Lohnsubventionen an Arbeitnehmer auf der Arbeitsangebotsseite an. Sie stellen einen Zuschuß auf den Lohnsatz dar. Marktlöhne unterhalb eines festgelegten Ziellohnsatzes bzw. Break-Even-Punktes werden um einen bestimmten Prozentsatz der Differenz zwischen eigentlichem Lohn und Ziellohn angehoben. Mit steigendem Lohnsatz verringert sich somit der an den Arbeitnehmer ausgezahlte Subventionsbetrag. Eine derartige Konstruktion wird bei Lohnsubventionen an Arbeitgeber i.d.R. vermieden, um nicht einen Anreiz für die Firmen zu schaffen, die Löhne künstlich niedrig zu halten. Lohnerhöhungen würden nämlich nur dazu führen, daß die Unternehmen weniger Subventionen erhalten. Lohnsubventionen an Arbeitnehmer veranlassen diese, ihr Anspruchslohnniveau nach unten zu korrigieren. Arbeitnehmer sind dazu bereit, Anstellungen zu niedrigeren Marktlöhnen zu akzeptieren. Lohnsubventionen an Arbeitnehmer sind also ein geeignetes Instrument zur Beschäftigungsexpansion, wenn Arbeitnehmer aufgrund zu geringer Marktlöhne keine Arbeitsverhältnisse eingehen. Durch die Subvention werden die von den Unternehmen gezahlten Löhne auf ein für die Arbeitnehmer akzeptables Niveau angehoben. Die Folge ist eine Erhöhung des Arbeitsangebotes über den subventionierten Lohnbereich. Um eine nachhaltige Änderung des Angebotsverhaltens auszulösen, sind Lohnsubventionen an Arbeitnehmer häufig permanent.

Es stellt sich jedoch die Frage, ob und inwieweit sich beide Subventionsmöglichkeiten unterscheiden. Ist der Arbeitnehmer Empfänger einer Lohnsubvention, welche er beispielsweise in Form von Gutscheinen („vouchers") an das inn einstellende Unternehmen weitergibt, dann besteht eine allokationstheoretische Indifferenz zwischen diesen beiden Varianten. Offenbart hingegen der Arbeitnehmer seinen Subventionsbezug gegenüber dem Unternehmen nicht, so liegt ein zur negativen Einkommensteuer analoger Fall vor, der hier nicht mehr eigens behandelt werden muß. Im Falle vollkommener polypolistischer Märkte spielt dies keine Rolle. Bei Existenz anderer Marktgegebenheiten ist zwischen den beiden Adres- 
saten zu unterscheiden. Hinzukommt, daß die Vorteile, die für eine Arbeitnehmersubvention sprechen mögen, zwar theoretisch begründet sind. Die Festlegung auf Arbeitgeber oder Arbeitnehmer muß jedoch auch in Abhängigkeit von den institutionellen Gegebenheiten gesehen werden. Insbesondere ist hier auch auf ein kostengünstiges Vergabeverfahren im Rahmen der gegebenen steuer- und/oder sozialpolitischen Arrangements zu achten. Geht die Subvention beispielsweise aus verwaltungstechnischen Gründen an den Arbeitgeber, so müssen die Verträge derartig gestaltet werden, daß der tatsächliche Empfänger auch der Arbeitnehmer ist. Diese Vorgehensweise entspricht der durchaus üblichen Praxis von Besteuerungsverfahren. In der Literatur identifizierte und oben diskutierte Vorteile beider Subventionsformen können soweit möglich miteinander verknüpft werden, was in den im folgenden Abschnitt ausgewählten Charaktieristika der Lohnsubvention umgesetzt wird.

\subsection{Auswahl der Subventionsvariablen}

Der voranstehende Abschnitt zeigte die Anzahl und Variationsmöglichkeiten der für ein Lohnsubventionsmodell notwendigen Variablen. Die in dieser Studie vorgeschlagenen Variablen und Gestaltungsparameter wurden auf der Grundlage der Analyse der Kapitel 2 und 3 festgelegt und werden an dieser Stelle nicht mehr ausführlich begründet. Hervorzuheben sind die Anreizfunktion, die Transparenz im Sinne einer einfachen und auf die Zielgruppe bezogen generellen Regelung, sowie die Dauerhaftigkeit der Förderung ohne Bindung an spezifische Eigenschaften der Arbeitgeber oder der ausgeübten Tätigkeit.

Bevor in Kapitel 4.2.2 die konkrete Auswahl der Modellparameter vorgestellt wird, bietet es sich an, den breiteren Rahmen aufzuspannen, in welchen sich aus systematischer und konzeptioneller Sicht das im folgenden diskutierte Modell langfristig einordnen sollte. 


\subsubsection{Der Rahmen: Zielführende Integration von Arbeitslosenhilfe und Sozialhilfe}

Die Darlegung der theoretischen Grundlagen dieser Arbeit in Kapitel 2 hat gezeigt, daß sich die Struktur wie die Ursachen der Arbeitslosigkeit in Deutschland spätestens seit Mitte der achtziger Jahre grundlegend verändert haben. Bei der Konzeptionierung und Schaffung des Bundessozialhilfegesetzes (1962) und des Arbeitsförderungsgesetzes (1969) war Langzeitarbeitslosigkeit ebensowenig bedeutend wie ein Zusammenhang von Sozialhilfebezug und Arbeitslosigkeit als Ursache erkennbar waren. Die Ausgestaltung der Absicherung gegen das Risiko Erwerbslosigkeit durch Arbeitslosengeld und Arbeitslosenhilfe war somit völlig unabhängig von der subsidiären Sicherung durch das Sozialhilfesystem.

Diese Situation hat sich grundlegend geändert, und mit der kontinuierlich und zunächst auch weiterhin steigenden Langzeitarbeitslosigkeit ist Arbeitslosigkeit zu einem immer bedeutenderen Grund für Sozialhilfebedürftigkeit, d.h. für Armut geworden. Vor diesem Hintergrund muß das Nebeneinander der angesprochenen Sicherungssysteme überdacht und - ausgerichtet auf das Ziel der Wiedereingliederung von Langzeitarbeitslosen in die Erwerbsgesellschaft neu organisiert werden. Für eine Integration von Arbeitslosen- und Sozialhilfe sprechen dabei sowohl theoretische als auch praktische, d.h. erfahrungsgeleitete Gründe.

Zunächst ist aus theoretischer Perspektive anzumerken, daß die Bekämpfung eines Problems (hier: Langzeitarbeitslosigkeit) mit zwei Instrumenten (Arbeitslosen- und Sozialhilfe) für den gleichen Personenkreis ineffizient in bezug auf Mitteleinsatz und ineffektiv in bezug auf die Zielerreichung ist. Konsequenzen dieser Parallelität zweier konkurrierender und aus Steuermitteln finanzierter Systeme sind beispielsweise die bereits angesprochene "Verschiebepolitik" durch einjährige sozialversicherungspflichtige Beschäftigung durch die Sozialhilfeträger und die Intransparenz durch grundlegend verschiedene, weil unterschiedlichen Konzepten folgende, Regelungen in bezug auf Zumutbarkeit, Anrechnungsvorschriften, Vermögenslage oder Rückgriff auf Angehörige. 
Auf der praktischen Ebene zeigt sich die Problematik in Koordinationsproblemen und Reibungsverlusten zwischen den zuständigen Behörden, die durch anhaltend hohe Arbeitslosigkeit und Budgetrestriktionen verstärkt werden. Entsprechend konzentrieren die Arbeitsämter ihre Eingliederungsbemühungen auf die Bezieher von Arbeitslosengeld. Diese Vorgehensweise ist effektiv, da Kurzzeitarbeitslose leichter zu vermitteln sind und so - bei begrenzten Ressourcen - die Vermittlungsquoten erhöht werden können. Hinzu kommt, daß die Arbeitsämter in der Regel nicht über die Erfahrungen und Qualifikationen verfügen, mehrdimensionale Vermittlungshemmnisse bei Langzeitarbeitslosen abzubauen und die stark standardisierten Maßnahmen multiple Hemmnisse nicht berücksichtigen. Sozialämter sind wiederum nur für einen kleinen Teil der Arbeitslosenhilfeempfänger zuständig ${ }^{18}$ und personell überfordert. Diese Praxis führt zu der in Öffentlichkeit wie Wissenschaft wenig beachteten Tatsache, daß auf die Bezieher von Arbeitslosenhilfe die geringsten Vermittlungsbemühungen verwandt werden.

Für eine strukturelle Reformierung der Sicherungssysteme gegen Erwerbslosigkeit spricht darüberhinaus, daß - wie an anderer Stelle bereits angesprochen - die Vielfalt an neuen Instrumenten und Maßnahmen, die auf allen Ebenen in den letzten Jahren entwickelt wurden, nur marginale Erfolge bei der Eindämmung von Langzeitarbeitslosigkeit gezeigt haben.

Es kann sich hier sicherlich nicht um eine unkompensierte Abschaffung der Arbeitslosenhilfe und die schlichte Absenkung - soweit es individuell dazu kommt - der Leistungsansprüche auf Sozialhilfeniveau handeln. Gleichwohl können individuell verschlechterte Leistungsansprüche kein eine Reform ausschließendes Pauschalargument darstellen, wie teilweise in der Literatur angeführt (vgl. Bäcker/Hanesch 1997).

Es ist unbestritten, daß sich aufgrund der unterschiedlichen Konzeptionen der Arbeitslosenhilfe mit dem Lohnbezug und der Sozialhilfe mit dem Bedarfsprinzip bei einer Integration beider Systeme Probleme in bezug auf die Abstimmung von Anrechnungsvorschriften und Anreizproblemen oder die anzupas-

${ }^{18}$ Für quantitative Aussagen siehe die Berechnungen in Kapitel 6. 
sende Finanzausstattung der Leistungsträger ergeben. Diese Probleme erscheinen jedoch lösbar und sollten angesichts der Problematik steigender Langzeitarbeitslosigkeit kein Reformhemmnis darstellen. Denkbar sind kompensatorische Maßnahmen beim Arbeitslosengeld beispielsweise in bezug auf die Dauer des Leistungsbezugs, Übergangsregelungen wie eine Befristung des Bezugs von Arbeitslosengeld oder die Beibehaltung der im Vergleich zur Sozialhilfe deutlich weniger restriktiven Anrechnungsvorschriften der Arbeitslosenhilfe für ehemalige Bezieher von AFG-Leistungen.

Ohne hier diese Diskussion weiter vertiefen zu können, sei auf zwei Aspekte hingewiesen, über die in der Literatur weitgehend Einigkeit besteht (vgl. Wilk 1997a):

- Die zur Bekämpfung der Probleme multipler Vermittlungshemmnisse bei Langzeitarbeitslosigkeit angemessene Ebene stellen aufgrund ihrer Erfahrungen, sozialpädagogischen Kompetenz und Bürgernähe die Sozialhilfeträger dar. Allerdings ist sicherlich im Hinblick auf eine erfolgreiche Vermittlung in den Ersten Arbeitsmarkt eine kritische Größe notwendig, die sich an den lokalen bzw. regionalen Wirtschaftsräumen orientieren sollte.

- Während die Ressourcen- und Ergebnisverantwortung in einer Hand, d.h. bei den Sozialhilfeträgern konzentriert sein sollte, bietet die Integration auch die Chance, die Effektivität der Eingliederungsbemühungen durch den Einbezug externer Dienstleister in den Betreuungs- und Vermittlungsprozeß zu erhöhen. "Vielmehr wird es darauf ankommen, daß sich die kostenverantwortliche Organisation des integrierten Sozialtransfers auf die Steuerung der Dienstleistungsprozesse einschließlich des Mitteleinsatzes als Kernaufgabe konzentriert und durchführende Dienstleistungen an dritte (private, gemeinnützige oder öffentliche) Organisationen delegiert. Dabei gelingt Steuerung am besten und in effizienter Weise, wenn zwischen den Dienstleistern ein echter Wettbewerb besteht" (Hartmann 1998, 11).

Insbesondere auf den letzten Aspekt wird bei dem zu entwickelnden Design für eine Beratungs-, Betreuungs- und Vermittlungsagentur (siehe Kapitel 5) detaillierter eingegangen. 


\subsubsection{Festlegung der Subventionsparameter}

Die Zielgruppe des Instruments sind die langzeitarbeitslosen Arbeitslosenhilfeund Sozialhilfeempfänger in Hessen, die dem Arbeitsmarkt zur Verfügung stehen, also erwerbsfähig sind. Die Abschätzung dieses angebotsseitigen Potentials erfolgt in Kapitel 6. Die Konzentration auf diese Gruppe erschließt sich aus ihrer besonderen Problematik infolge der in Deutschland vorherrschenden hysteresen Arbeitslosigkeit, wie sie im zweiten Kapitel skizziert wurde. Daneben bietet es sich an, ein derartiges Maßnahmenbündel in einem abgegrenzten Rahmen, wie es mit der Festlegung auf ein Bundesland der Fall ist, und mit einer klar definierten Zielgruppe zu starten. Gleichwohl ist der präsentierte Vorschlag regional wie personell erweiterbar und sollte im Hinblick auf eine langfristig anzustrebende bundesweite und allgemeine Lösung mit dem Ziel der Beschäftigungsförderung Geringqualifizierter verstanden werden. Zudem erscheint die Bundeslandebene als eine sinnvolle, die Koordinations- und Intransparenzprobleme weitesgehend lösende Ebene. Durch die Begrenzung auf Langzeitarbeitslose werden zudem Mitnahmeeffekte vermieden. Weitere Mitnahme- bzw. Drehtüreffekte können mit der Bedingung vermieden werden, daß freie Arbeitsplätze nur dann mit Personen aus der geförderten Zielgruppe besetzt werden, wenn sie zuvor beispielsweise mindestens drei Monate unbesetzt waren.

Die Lohnsubventionen selbst werden folgendermaßen gestaltet:

- Die Lohnsubvention setzt positive Arbeitsanreize, weil sie nur bei Aufnahme einer Enwerbsarbeit realisiert wird.

- Die Subvention erfolgt auf Stundenlohnbasis, um Teilzeitarbeit nicht zu diskriminieren $^{19}$.

- Ausgehend von einem bestimmten Mindestlohn erfolgt eine degressive Lohnsubvention, d.h. eine solche Lohnsatzsubvention führt bei steigendem Lohnsatz zu einem sinkenden Subventionsbedarf. Bei einem ebenfalls fest-

19 Sollte darüberhinaus eine besonders privilegierte Behandlung von freiwilliger Teilzeitarbeit angestrebt werden, empfiehlt sich ein Bezug auf Monats- oder Jahreslohnbasis. Dies wird hier aber nicht angestrebt. 
zulegenden Höchstlohnsatz läuft die Subvention aus. Insgesamt bleibt für den einzelnen der Anreiz, mehr zu verdienen und aus der Subventionszone „hinauszuwachsen“, erhalten. Gleichzeitig wird kollusives Verhalten zu Lasten des Subventionsträgers vermieden.

- Ein Mindestlohn ist festzusetzen, um einerseits sittenwidrige Verträge und andererseits Absprachen zwischen Arbeitgebern und Arbeitnehmern zuungunsten des Staates zu vermeiden.

- Die Laufzeit der Subvention ist unbegrenzt, wodurch insbesonders die Motivation der Arbeitnehmer erhöht und Humankapitalinvestitionen angeregt werden.

- Gefördert werden Vollzeitbeschäftigungsverhältnisse bzw. Teilzeitbeschäftigungsverhältnisse mit einer Mindeststundenzahl von 19 Wochenstunden ${ }^{20}$.

- Bezugsperson für die Subvention ist das Individuum; haushaltsbezogene Größen, wie sie in der Sozialhilfe berücksichtigt werden, werden aus Anreizgründen bei der Bestimmung der Subventionshöhe einbezogen ${ }^{21}$. Es findet keine Bedürftigkeitsprüfung statt, da diese bereits Voraussetzung zum Bezug von Arbeitslosenhilfe bzw. Sozialhilfe war.

- Die Höhe der Subvention orientiert sich an den besetzten unteren Lohngruppen im Bereich der Branchen, die für zusätzliche Beschäftigungspotentiale in Frage kommen.

- Trotz der 0.a. theoretischen Vorteile einer arbeitnehmerbezogenen Ausgestaltung sollte der Arbeitgeber Subventionsempfänger sein, da die Abwicklung im Rahmen des Lohnsteuerverfahrens innerhalb eines solchen Modellversuches erhebliche administrative Erleichterung mit sich bringt ${ }^{22}$.

Im Sinne einer Förderung von Teilzeit- bzw. von Mehrfachbeschäftigung von Arbeitnehmern bei verschiedenen Arbeitgebern ist in einem späteren Schritt ein Wegfall der Mindeststundenzahl zu bedenken.

Die Notwendigkeit zur Berücksichtigung der Haushaltsgröße ergibt sich im wesentlichen aus der Differenz von Kindergeld und entsprechenden Sozialhilfebezügen sowie der geringeren Steuerersparnis in der Splittingtabelle bei niedrigen Einkommen.

22 Durch die einfache Abwicklung und Verrechnung mit der Steuerschuld durch den Arbeitgeber entstehen praktisch keine Kosten durch die Auszahlung der Lohnsubvention. 
- Das gesamte Arbeitseinkommen, also Lohn bzw. Gehalt und Subvention, ist steuer- und sozialversicherungspflichtig. Die Anrechnungsvorschriften für Leistungsbezug bleiben hier zunächst unberührt.

- Eine Zusätzlichkeitsprüfung für subventionierte Arbeitsverhältnisse entfällt, da diese nur im Ersten Arbeitsmarkt placiert werden und der Phantasie des Marktes keine Grenzen gezogen werden sollen.

- Erwerbsfähigen Leistungsbeziehern, die sich aktiv einer Teilnahme an der Beratungs-/Betreuungs- und Vermittlungsmaßnahme (s. Kapitel 5) entziehen, werden entsprechend der gültigen Bestimmungen die Regelleistungen um $25 \%$ gekürzt.

\subsection{Modellstruktur und-berechnungen}

Entscheidungen zugunsten bestimmter Mindestniveaus und Förderungsgrößen sind in dem Zusammenhang dieser Studie immer eher politisch und weniger ökonomisch motiviert. Grundlegend für die folgenden Werte sind zum einen die weitgehende Vermeidung der Sozialhilfefalle und zum anderen die Orientierung an den unteren Lohngruppen. Die institutionell bedingte Spannbreite der Lohnsubvention zwischen der existenzminimumsichernden Sozialhilfe und den unteren besetzten Lohngruppen begrenzt die Anreizwirkungen dieses Instruments. Diese Tatsache kann aber im Rahmen dieser Studie nur als modellexogene Randbedingung betrachtet werden. Aus diesen Überlegungen folgt, daß ein geförderter Arbeitnehmer mindestens ein Stundeneinkommen von $10 \mathrm{DM}$ erhalten sollte. Dies entspricht in etwa den niedrigsten Verdienstmöglichkeiten in den unteren Lohngruppen (WSI 1998). Der Mindestlohn für Unternehmen liegt bei $6 \mathrm{DM}$, die Abschmelzrate der Stundenlohnsubvention bei $50 \%{ }^{23}$ und die Förderobergrenze bei $14 \mathrm{DM}$. Die folgende Tabelle faßt die verschiedenen Größen im Förderbereich für einen alleinstehenden Arbeitnehmer zusammen:

D.h. 0,50 DM Subventionsentzug bei 1 DM Stundenlohnzuwachs. 


\begin{tabular}{|c|c|c|c|c|c|c|}
\hline \multirow{2}{*}{$\begin{array}{l}\text { Lohn } \\
(\mathrm{DM} / \mathrm{h})\end{array}$} & \multirow{2}{*}{$\begin{array}{l}\text { Subven- } \\
\text { tion (DM/h) }\end{array}$} & \multirow{2}{*}{$\begin{array}{c}\text { Einkommen } \\
(\mathrm{DM} / \mathrm{h})\end{array}$} & \multirow{2}{*}{$\begin{array}{l}\text { Lohnauf- } \\
\text { schlag (\%) }\end{array}$} & \multirow{2}{*}{$\begin{array}{l}\text { Bruttomonats- } \\
\text { einkommen }{ }^{*} \text {. }\end{array}$} & \multicolumn{2}{|c|}{ Arbeitskosten (monatl.) } \\
\hline & & & & & o. Soz.Abg. & mit Soz.Abg. \\
\hline 6 & 4,0 & 10,0 & 66,67 & 1.620 & 972 & $1.176,1$ \\
\hline 7 & 3,5 & 10,5 & 50,00 & 1.701 & 1.134 & $1.372,1$ \\
\hline 8 & 3,0 & 11,0 & 37,50 & 1.782 & 1.296 & $1.568,2$ \\
\hline 9 & 2,5 & 11,5 & 27,78 & 1.863 & 1.458 & $1.764,2$ \\
\hline 10 & 2,0 & 12,0 & 20,00 & 1.944 & 1.620 & $1.960,2$ \\
\hline 11 & 1,5 & 12,5 & 13,64 & 2.025 & 1.782 & $2.156,2$ \\
\hline 12 & 1,0 & 13,0 & 8,33 & 2.106 & 1.944 & $2.352,2$ \\
\hline 13 & 0,5 & 13,5 & 3,85 & 2.187 & 2.106 & $2.548,3$ \\
\hline 14 & 0,0 & 14,0 & 0,00 & 2.268 & 2.268 & $2.744,3$ \\
\hline
\end{tabular}

*) Vor Lohnsteuer und Sozialversicherungsabgaben

Tabelle 1: Subvention, Brutto-Monatseinkommen und Arbeitskosten in Abhängigkeit vom Stundenlohn

Graphisch läßt sich das Einkommen eines Alleinstehenden in Abhängigkeit vom Stundenlohn folgendermaßen darstellen:

\section{Modell der Lohnsubvention (Alleinstehender)}

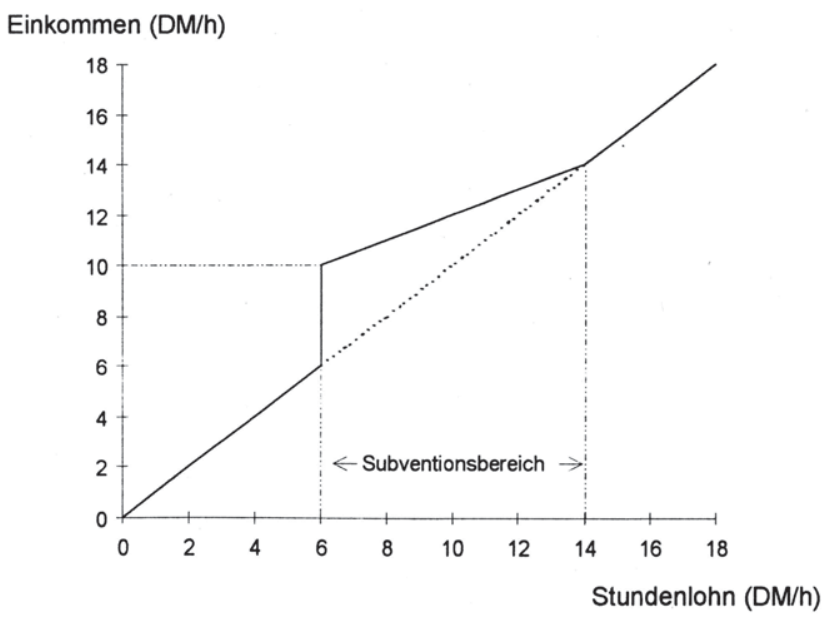

Abbildung 1: Bruttostundenlohn eines Alleinstehenden mit Lohnsubvention in Abhängigkeit vom Stundenlohn

Bei einer tariflichen Wochenarbeitszeit von 38 Stunden würde das mit Hilfe der Subvention erreichbare Bruttoeinkommen zwischen 1.620 DM und 2.268 DM 
liegen, während die entsprechenden vom Arbeitgeber zu entrichtenden Bruttolöhne zwischen 972 DM und eben 2.268 DM liegen ${ }^{24}$.

Da die Einkommen aufgrund steuer- und sozialpolitischer Überlegungen vollständig steuer- und sozialversicherungspflichtig sind, würden sich bei dieser Form der Subventionierung die Kostenvorteile der Arbeitgeber ebenso wie die Anreizwirkung für den Arbeitnehmer um deren respektive Anteile an der $\mathrm{Fi}$ nanzierung der Sozialversicherungen erheblich verringern. Im Falle des Mindesteinkommens beträgt die Lohnsubvention $648 \mathrm{DM}$ (siehe nächste Tabelle). Der zusätzliche Arbeitgeber- bzw. Arbeitnehmeranteil infolge der Lohnsubvention beträgt dann jeweils 136 DM. Zur Erhaltung der Anreizwirkungen sowohl auf Arbeitgeber- als auch auf Arbeitnehmerseite sollten die zusätzlichen Sozialversicherungsbeiträge infolge des Lohnaufschlages bezuschußt werden. Die Sozialversicherungsbeiträge für den nichtbezuschußten Einkommensbestandteil sind von Arbeitnehmer und Arbeitgeber zu tragen. Aus diesen Daten lassen sich nun die monatlichen Subventionsbeträge je Subventionshöhe und alleinstehender Erwerbsperson, wie sie in der nachfolgenden Tabelle abgetragen sind, berechnen.

\begin{tabular}{|c|c|c|c|}
\hline $\begin{array}{c}\text { Lohn } \\
(\mathrm{DM} / \mathrm{h})\end{array}$ & $\begin{array}{c}\text { Lohn- } \\
\text { subvention }\end{array}$ & $\begin{array}{c}\text { Beitrags- } \\
\text { subvention }\end{array}$ & $\begin{array}{c}\text { Gesamt- } \\
\text { subvention }\end{array}$ \\
\hline 6 & 648 & 272,16 & 920,16 \\
\hdashline 7 & 567 & 238,14 & 805,14 \\
\hdashline 8 & 486 & 204,12 & 690,12 \\
\hdashline 9 & 405 & 170,10 & 575,10 \\
\hdashline 10 & 324 & 136,08 & 460,08 \\
\hdashline 11 & 243 & 102,06 & 345,06 \\
\hdashline 12 & 162 & 68,04 & 230,04 \\
\hdashline 13 & 81 & 34,02 & 115,02 \\
\hdashline 14 & 0 & 0,00 & 0,00 \\
\hline
\end{tabular}

Tabelle 2: Subventionshöhen in Abhängigkeit von der Stundenlohnhöhe

Für einen alleinstehenden Arbeitnehmer ergäben sich bei dem geringsten Stundenlohn von 6 DM und einem Bruttoeinkommen von 1620 DM Sozialversi-

24 In Anlehnung an Berechnungen in der Literatur liegt den Zahlen zum Monatseinkommen eine monatliche Arbeitszeit von 162 Stunden für Vollzeitbeschäftigung zugrunde (vgl. Scharpf 1994). 
cherungsabgaben in Höhe von 204 DM, Lohnsteuer in Höhe von 62,50 DM²5 und ein Nettoeinkommen in Höhe von 1.353,50 DM. Auch bei diesem niedrigsten Stundenlohn verbleibt - selbst bei ungünstigster Rechnung - eine Anreizwirkung gegenüber einem Sozialhilfebezug, der bei einem alleinstehenden Arbeitnehmer maximal 1213 DM monatlich betragen kann ${ }^{26}$. Die Anreizwirkung auch im untersten Entlohnungsbereich wird noch deutlicher, wenn man einerseits die - im Falle der Ablehnung einer vermittelten Arbeitsstelle - Kürzung des Regelsatzes um 25\% (d.h. 135 DM) und andererseits den aufgrund des niedrigen Einkommens auch bei Erwerbstätigkeit bestehenden Wohngeldanspruch berücksichtigt. In diesem Fall liegen die monatlichen Nettoeinkommen bei 1078 DM bei Sozialhilfebezug und bei 1501,50 DM bei Erwerbstätigkeit ${ }^{27}$ und einem Stundenlohn (ohne Subvention) von 6 DM. Aus Sicht des Arbeitgebers wird die Anreizwirkung ebenfalls deutlich: Um für seinen Mitarbeiter ein Nettoeinkommen von 1350 DM pro Monat ohne Subvention zu erreichen entstünden inm Arbeitskosten inklusive Sozialabgaben von etwa 2500 DM monatlich gegenüber etwa 1180 DM (siehe obige Tabelle) im Falle des Modells der Lohnsubvention.

Aufgrund der Differenz von Kindergeld und existenzminimumsicherndem Sozialhilfebezug sowie der erst bei 24120 DM Jahreseinkommen einsetzenden Splittingtabelle ist die Berücksichtigung der Haushaltsgröße zur Aufrechterhaltung der Anreizwirkung notwendig. Die Stundenlohnsubvention erhöht sich dann um $50 \%$ bei nichterwerbstätigem Ehepartner und um $25 \%$ für jedes nichterwerbstätige Kind bezogen auf die höchste Förderung von $4 \mathrm{DM}$ je Stunde, d.h. 2 DM für den Ehepartner und 1 DM für ein Kind je Arbeitsstunde. Der Zuschlag von $25 \%$ je Kind deckt dabei die Differenz zwischen durchschnittlichem Sozialhilfebezug und Kindergeld während der Faktor 0,5 die zu-

25

\section{abz}

26

27

Der Wohngeldanspruch bei einem Bruttoeinkommen von 1620 und abzugsfähigen 30\% aufgrund der Sozialabgaben liegt zwischen 12 und 148 DM abhängig von der Mietbelastung. Wie bei der Berechnung des maximalen Sozialhilfebezuges wurde zur Vergleichbarkeit das höchstmögliche Wohngeld angesetzt (Basis: Presse- und Informationsamt der Bundesregierung 1997). 
sätzlichen Haushaltsausgaben beim Übergang vom Ein- zum Zweipersonenhaushalt im Sinne des Konzepts der Äquivalenzeinkommen abbildet. Für einen Haushaltsvorstand mit einem Ehepartner und einem Kind ergibt sich bei einem Lohn von 6 DM je Stunde eine Subvention von 7 DM je Stunde (siehe nachfolgende Tabelle). Bei steigenden Löhnen wird die Subvention dann abgeschmolzen und fließt hier - bei der Abschmelzrate 0,5 - bei einem Stundenlohn von 20 DM aus. Die Abschmelzung erfolgt damit analog zum in den USA im Niedriglohnbereich sehr erfolgreichen Instrument des Earned Income Tax Credit (EITC).

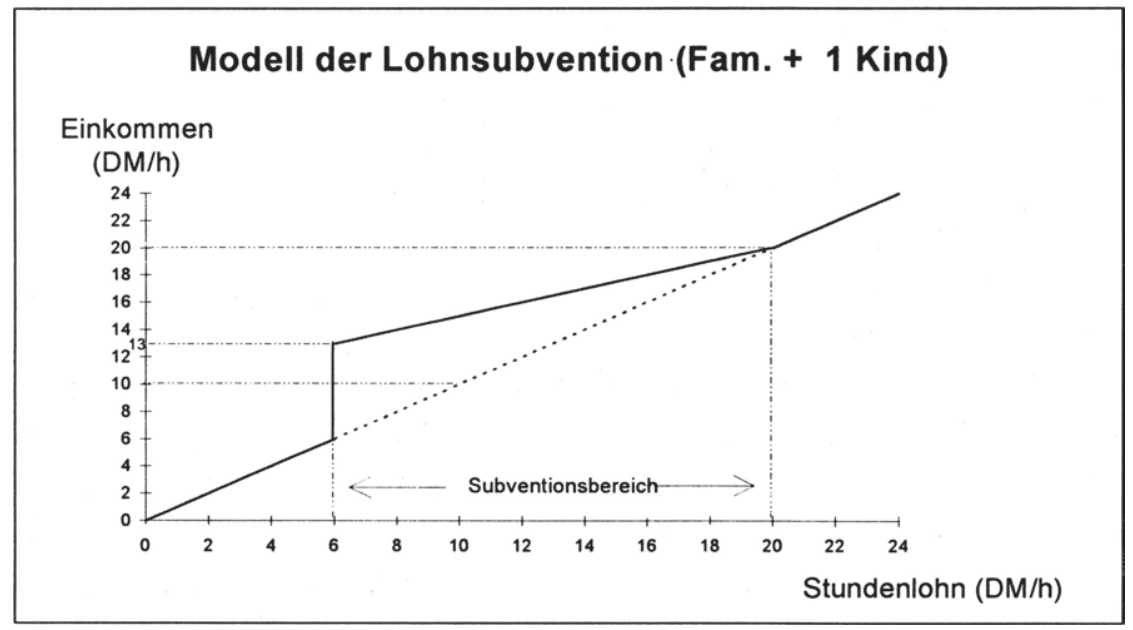

Abbildung 2: Bruttostundenlohn eines Alleinstehenden mit Lohnsubvention in Abhängigkeit vom Stundenlohn 


\begin{tabular}{|c|c|c|c|c|c|c|}
\hline $\begin{array}{l}\text { Lohn } \\
(\mathrm{DM} / \mathrm{h})\end{array}$ & $\begin{array}{c}\text { Grundsub- } \\
\text { vention (DMh) }\end{array}$ & $\begin{array}{l}\text { Haushaltszu- } \\
\text { schlag (DM/h) }\end{array}$ & $\begin{array}{c}\text { Einkom- } \\
\text { men }\end{array}$ & $\begin{array}{l}\text { Bruttomonats- } \\
\text { einkommen }{ }^{*} \text { ) }\end{array}$ & $\begin{array}{l}\text { Arbeitskost } \\
\text { o.Soz.Abg. }\end{array}$ & $\begin{array}{l}\text { en (monatl.) } \\
\text { mit Soz.Abg. }\end{array}$ \\
\hline 6 & 4,0 & 3 & 13,0 & 2.106 & 972 & $1.176,1$ \\
\hline 7 & 3,5 & 3 & 13,5 & 2.187 & 1.134 & $1.372,1$ \\
\hline 8 & 3,0 & 3 & 14,0 & 2.268 & 1.296 & $1.568,2$ \\
\hline 9 & 2,5 & 3 & 14,5 & 2.349 & 1.458 & $1.764,2$ \\
\hline 10 & 2,0 & 3 & 15,0 & 2.430 & 1.620 & $1.960,2$ \\
\hline 11 & 1,5 & 3 & 15,5 & 2.511 & 1.782 & $2.156,2$ \\
\hline 12 & 1,0 & 3 & 16,0 & 2.592 & 1.944 & $2.352,2$ \\
\hline 13 & 0,5 & 3 & 16,5 & 2.673 & 2.106 & $2.548,3$ \\
\hline 14 & 0,0 & 3 & 17,0 & 2.754 & 2.268 & $2.744,3$ \\
\hline 15 & $-0,5$ & 3 & 17,5 & 2.835 & 2.430 & $2.940,3$ \\
\hline 16 & $-1,0$ & 3 & 18,0 & 2.916 & 2.592 & $3.136,3$ \\
\hline 17 & $-1,5$ & 3 & 18,5 & 2.997 & 2.754 & $3.332,3$ \\
\hline 18 & $-2,0$ & 3 & 19,0 & 3.078 & 2.916 & $3.528,4$ \\
\hline 19 & $-2,5$ & 3 & 19,5 & 3.159 & 3.078 & $3.724,4$ \\
\hline 20 & $-3,0$ & 3 & 20,0 & 3.240 & 3.240 & $3.920,4$ \\
\hline
\end{tabular}

*) Vor Lohnsteuer und Sozialversicherungsabgaben

Tabelle 3: Grundsubvention, Haushaltszuschlag, Buttoeinkommen und Arbeitskosten bei einer Familie mit $1 \mathrm{Kind}$

Im Falle des niedrigsten Stundenlohns von 6 DM, unverändert 204 DM Sozialversicherungsabgabe ${ }^{28}$ und Kindergeld von 220 DM liegt das Nettoeinkommen bei $2.122 \mathrm{DM}^{29}$. Es liegt damit zwar leicht unter dem maximalen Sozialhilfebezug von $2.261 \mathrm{DM}^{30}$, zu berücksichtigen ist aber, daß es sich hierbei um den theoretisch ungünstigsten Fall handelt und daß bei Nichtaufnahme einer angebotenen Tätigkeit dauerhaft 25\% des Eckregelsatzes des Haushaltsvorstandes entzogen werden können. Analog zur Berechnung für einen alleinstehenden Arbeitnehmer ergibt sich dann ein Nettoeinkommen von 2.126 DM bei Sozialhilfebezug und $2.523 \mathrm{DM}^{31}$ bei Enwerbstätigkeit. Die Anreizwirkung wird noch deutlicher, wenn man berücksichtig, daß in letzterem Fall Ansprüche an die

Die zusätzlichen Sozialversicherungsabgaben auf den Haushaltszuschlag werden analog zur Grundsubvention aus den genannten Gründen subventioniert.

29 Analog der Berechnung für einen Alleinstehenden. Steuern sind nach der Splittingtabelle nicht fällig.

30 Haushaltsvorstand $541 \mathrm{DM}$, nichterwerbstätiger Ehepartner $433 \mathrm{DM}$, nichterwerbstätiges Kind zwischen 8-14 Jahren 352 DM, maximales Wohngeld 799 DM und Kleidergeld 136 DM (Basis: Sozialamt Stadt Darmstadt).

31 Bei drei Haushaltsmitgliedern und einem Bruttoeinkommen von 2106 DM (abzüglich 30\% für die Sozialabgaben) ergibt sich ein Wohngeldanspruch von 11 bis 401 DM abhängig von der Mietbelastung (Basis: Presse- und Informationsamt der Bundesregierung 1997). 
Sozialversicherungen erworben werden. Der Haushaltszuschlag kann dabei je Haushalt nur einmal gewährt werden, d.h. im Falle zweier erwerbstätiger Erwachsener und einem nicht erwerbstätigen Kind verringert sich der Zuschlag von $75 \%$ auf $25 \%$, bezogen auf die Grundsubvention von 4 DM je Arbeitsstunde.

Weitergehende Berechnungen sind nur sehr eingeschränkt möglich, da - wie weiter hinten zu sehen - nur Angebots- und Nachfragepotentiale geschätzt werden können. Die tatsächliche Inanspruchnahme und die Verteilung der Löhne und Einkommen können jedoch nicht prognostiziert werden. Die fiskalischen Kosten dieses Modells der Lohnsubvention sind aufgrund der Konzentration auf Langzeitarbeitslose sehr begrenzt bzw. fallen nur durch die Begleitung der Subventionsmaßnahme durch eine Beratungs-, Betreuungs- und Vermittlungsagentur (s. Kapitel 5) an. Der maximale Subventionsbetrag bei einem Alleinstehenden beträgt 920 DM, bei einer Familie mit einem Kind 1610 $\mathrm{DM}^{32}$ und liegt damit jeweils unterhalb der Sozialhilfebezüge. Nettokosten könnten sich durch Mitnahmeeffekte lediglich dann ergeben, wenn ein Langzeitarbeitsloser auch ohne die Subvention eine Tätigkeit aufgenommen hätte, die zum Rückgang oder Wegfall von Leistungsbezügen geführt hätte. Dieser Personenkreis ist jedoch sehr begrenzt, so daß bei sehr vorsichtiger Schätzung für die Lohnsubvention von fiskalischer Neutralität ausgegangen werden kann.

Die Lohnsubvention ist geeignet, zur Zurückgewinnung von Tätigkeiten mit geringer Produktivität, die dem Arbeitsmarkt verlorengegangen sind, beizutragen, insbesondere aber bei der Etablierung neuer Beschäftigungsfelder. Hierdurch wird zudem anerkannt, daß nicht den Arbeitgebern die Last sozial definierter Mindestlöhne aufgeladen werden kann, sondern daß es Arbeitnehmer gibt und weiterhin geben wird, deren Qualifikation unzureichend für auskömmliche Marktlöhne ist. Insofern kann in der Lohnsubvention ein Ansatz zu einer „sozialen Beschäftigungspolitik“ (Vierling 1997, 649) gesehen werden, da Beschäftigungs- und Perspektivenzuwächse am „unteren Ende“ der qualifikatorischen Schichtung der Gesellschaft erzielt werden können, dort wo der mengen- und anteilsmäßige Schwerpunkt der Arbeitslosigkeit liegt. Da aufgrund

\footnotetext{
${ }^{32} 7$ DM Subvention je Stunde zuzüglich $42 \%$ Gesamtsozialversicherungsbeitrag.
} 
vielfacher Vermittlungshemmnisse der meisten Langzeitarbeitslosen mit fiskalischen Anreizen allein nur selten ein erfolgreicher und vor allem dauerhafter (Wieder-)Einstieg in die Erwerbsarbeit gelingen kann, werden im folgenden Kapitel die notwendigen Beratungs-, Begleitungs- und Vermittlungsleistungen eines landesweiten Programms skizziert. 


\section{Beratungs-, Betreuungs- und Vermittlungsagentur: „Jobs für Langzeitarbeitslose“}

\subsection{Grundidee und Rahmenbedingungen}

Die Evaluation der bisherigen Erfahrungen bestehender Beschäftigungsinitiativen zeigte, daß das allgemeine Instrument der Lohnsubvention durch individuell bezogene Beratungs-, Betreuungs- und Vermittlungstätigkeiten ergänzt werden muß, soll eine optimale Eingliederung der Zielgruppe in den Ersten Arbeitsmarkt erfolgen. Diese Zielvorgabe wiederum ist notwendig, um eine dauerhafte Entlastung aller beteiligten staatlichen Haushalte und hier vor allem der Kommunen zu gewährleisten. Hinsichtlich der Zielgruppe erscheint eine zusätzliche personenbezogene und mithin biographisch orientierte Beschäftigungsförderung notwendig, denn „( $m$ )it der bloßen Vermittlung auf einen neuen Arbeitsplatz ist es nicht getan. Die psycho-sozialen Deprivationen verhindern die Aufnahme einer neuen Arbeitsstelle entweder gleich oder sie haben das Arbeitsvermögen so nachhaltig gestört, daß eine (erneute) Kündigung droht" (Hackenberg/Wagner 1996, 777). Entsprechend sollte die Förderung und Forderung von Eigenaktivität auf Seiten der Teilnehmer, verbunden mit dem Ziel deren Selbstbewußtsein aufzubauen, ein zentrales Anliegen dieser Agenturen sein. Der Erfolg der hier vorgeschlagenen Agenturen läßt sich folglich nicht nur an der Entlastung der Sozialhaushalte, sondern auch und gerade an der Verringerung der psychosozialen und medizinischen Kosten messen. Ihre Arbeit besitzt somit nicht nur eine finanz- und beschäftigungspolitische, sondern ebenso eine sozialpolitische Dimension. Um die Mitglieder der Zielgruppe auf die Arbeitswelt vorzubereiten, erscheint aufgrund der vorliegenden Erfahrungen ein Zeitraum von durchschnittlich acht Wochen als ausreichend. Die Teilnahme an diesem Coaching sollte verpflichtend sein und eine aktive Verweigerung sollte dementsprechend zu einem Abschlag von $25 \%$ des Eckregelsatzes führen. 


\subsection{Aufgaben und Instrumente}

\subsubsection{Beratung}

Die erste und wichtigste Aufgabe der Agenturen ist die persönliche Beratung der Zielgruppenmitglieder. Ziel der Beratungsgespräche sollte ein individuelles Stärken-Schwächen-Profil sein, das als Grundlage für die weitere Förderung der jeweiligen Person, aber auch als erste vertrauensbildende Maßnahme zwischen Berater und Arbeitssuchenden zu verstehen ist.

In einem ersten Schritt müssen die persönlichen Präferenzen, Stärken, Interessen und Schwächen der Individuen eruiert werden. Herauszufinden sind insbesondere die verschiedensten vermittlungshemmenden Wettbewerbsnachteile, die beispielsweise in Schulden-, Sucht- oder Wohnungsproblemen, aber auch in psychischen Behinderungen oder allgemein fehlenden sozialen Kompetenzen zu sehen sind.

Auf der Grundlage dieses Stärken-Schwächen-Profils kann nun in Zusammenarbeit mit dem Arbeitssuchenden die weitere Vorgehensweise zur (Re)integration in den Arbeitsmarkt innerhalb der dafür zur Verfügung stehenden acht Wochen erarbeitet werden. In begründeten und begrenzten Einzelfällen kann eine längere Beratungs- und Betreuungsphase eingeräumt werden. Gleichzeitig dient das erarbeitete Profil dem Berater als Grundlage bei seinen Vermittlungsbemühungen. Bei entsprechender Eignung sollte eine Beratung auch die Möglichkeit einer selbständigen Erwerbstätigkeit fördern.

Die bedeutende Rolle und der damit verbundene Aufwand der Beratungsphase sollten aufgrund ihrer grundlegenden Position für alle weiteren Maßnahmen und für die damit zu schaffende Kooperationsbasis zwischen den Akteuren nicht unterschätzt werden. So waren diejenigen evaluierten Beschäftigungsinitiativen die erfolgreicheren, die in diesem Bereich einen ersten Schwerpunkt gesetzt hatten. 


\subsubsection{Betreuung}

Die Betreuung während der acht Wochen umfaßt zwei Bereiche: Zum einen werden stabilisierende sozialpädagogische und zum anderen berufsvorbereitende Maßnahmen durchgeführt.

Unter einer stabilisierenden Maßnahme ist die Beseitigung eines Vermittlungshemmnisses, welches nicht durch eine mangelnde Qualifikation des Arbeitssuchenden hervorgerufen wird, zu verstehen. Hierzu zählen sowohl therapeutische (Suchttherapie, Psychotherapie, psychologische Behandlung) als auch beratendende und organisatorische Maßnahmen (Schuldnerberatung, fehlende Kinderbetreuungsplätze, Wechsel in günstigere Wohnung). Gerade Langzeitarbeitslose verharren oftmals in einer resignativen und passiven Grundhaltung, aus der heraus sie keines ihrer bestehenden Probleme selbständig wirksam angehen können und wollen. Stabilisierende Maßnahmen erfordern bisweilen einen hohen personellen wie zeitlichen Aufwand, weswegen sie nicht zwingend innerhalb der Agentur ausgeführt werden müssen, sondern vielmehr in $\mathrm{Zu}$ sammenarbeit mit bereits bestehenden Institutionen durchgeführt werden sollten.

Zu den berufsvorbereitenden Maßnahmen zählen weniger berufsfachliche als vielmehr soziale Qualifizierungen sowie der Erwerb von Schlüsselqualifikationen. Hierzu gehört, daß die Arbeitnehmer wieder Basisqualifikationen wie Pünktlichkeit, Zuverlässigkeit, Fleiß, Ausdauer und Teamgeist erwerben. Als Methoden stehen Einzel- und Gruppenunterricht, aber auch das Training innerhalb eines Betriebspraktikums zur Verfügung. Betriebspraktika können in diesem Zusammenhang als Teil der Vermittlungsbemühungen betrachtet werden, da potentielle Arbeitgeber hierbei bereits Informationen über die möglichen zukünftigen Arbeitnehmer erhalten können. Vor dem Hintergrund der sich verändernden Arbeitswelt, aufbrechender Berufsbilder und der Tatsache, daß die überwiegende Zahl der potentiellen Arbeitsplätze im Dienstleistungbereich liegen dürfte, sollte die Rolle des Betriebspraktikums jedoch nicht überschätzt werden. Aus den gleichen Gründen wird auch auf berufsfachliche Maßnahmen verzichtet. Letztere haben prinzipiell statischen Charakter und würden die der 
Marktwirtschaft innewohnenden dynamischen Kräfte - hier in bezug auf die Evolution neuer Beschäftigungsfelder - zumindest stark relativieren.

Die Betreuungsleistung der Agentur endet allerdings nicht mit dem achtwöchigen Programm bzw. der Vermittlung in ein Arbeitsverhältnis. Vielmehr schließt sich eine beschäftigungsbegleitende Betreuung an, die je nach dem Grad der Schwere der individuellen Probleme zwischen 6 und 18 Monate dauern kann. Die Betreuung nach der Arbeitsaufnahme durch ein Tutonwesen dient der Stabilisierung des Beschäftigungsverhältnisses, um frühzeitige und leichtfertige Kündigungen von Arbeitnehmer- und Arbeitgeberseite zu verhindern. Gleichzeitig kann hierdurch der Kontakt zum Arbeitgeber gepflegt werden, was in Hinblick auf die Reputation und eine funktionierende Stellenakquise für eine Beschäftigungsinitiative von großer Bedeutung ist. Die Ex-post-Betreuung sollte auch für diejenigen Personen gelten, die den Sprung in die Selbständigkeit wagen.

\subsubsection{Vermittlung}

Die in diesem Programm involvierten Arbeitslosenhilfe- und Sozialhilfeempfänger sollten aufgrund der Logik des Dreiklangs von Beratung-BetreuungVermittlung einen Anspruch auf eine Vermittlung während der oder im Anschluß an die achtwöchige Coachingphase haben. Grundlage der Vermittlungsbemühungen sind die Ergebnisse von Beratung und Betreuung einerseits, aber auch das Bemühen der Agentur, wie es im nächsten Punkt skizziert werden soll.

\subsubsection{Akquise und Werbung}

Eine der wichtigsten Aufgaben bezüglich ihrer Effektivität und Außenwirkung dürfte die Akquise und Werbung für die Agenturen sein.

Die theoretische Analyse hat gezeigt, daß aufgrund der Veränderungen in der Arbeitswelt und der sektoralen Strukturverschiebungen in Richtung Dienstleistungsbranche eine latente Nachfrage nach Arbeitskräften vorhanden ist. Auf- 
gabe der Agenturen ist deshalb die Aktivierung dieser latenten Nachfrage, indem entsprechend der mit Hilfe internationaler Vergleiche berechneten Potentiale Betätigungsfelder aufgezeigt werden, mithin also neue Märkte geschaffen werden, und die potentiellen Arbeitgeber direkt angesprochen werden. Dieses aktive Joblobbying kann durch Kontakte oder Kooperation mit vorhandenen Institutionen wie der Industrie- und Handelskammer, der Handwerkskammer, dem Arbeitsamt, aber auch privaten Arbeitsvermittlern und Zeitarbeitsfirmen oder Vereinen forciert werden. Die Analyse der Beschäftigungsinitiativen hat gezeigt, daß die Zahl von Arbeitsplatzangeboten positiv mit dem Werben um eben diese Arbeitsplätze korreliert. Dies bestätigt die These einer latenten Nachfrage im Bereich niedrig qualifizierter und niedrig entlohnter Tätigkeiten, die geweckt werden will. Auch wenn diese Agenturen vor allem lokale und regionale Schwerpunkte haben, sollten die Möglichkeiten moderner Massenmedien zur Erreichung potentieller Arbeitgeber und hier vor allem privater Haushalte genutzt werden.

Die Akquisetätigkeit dient aber nicht nur der Schaffung neuer Märkte, sondern geht viel weiter darüber hinaus. Sie macht die Agentur im lokalen und regionalen Arbeitsmarkt bekannt. Über die Akquise kann die Agentur somit Werbung für sich und ihre Ziele sowie die von ihr zu vermittelnden Arbeitnehmer betreiben. Dies ist deshalb notwendig, da der Erfolg und die Akzeptanz derartiger Beschäftigungsinitiativen nicht nur, aber auch von ihrer Außenwirkung und ihrem Bekanntheitsgrad abhängen. Zu diesem Zweck sollte die Notwendigkeit und Wirkung von Marketinginstrumenten wie griffige Slogans, einheitliches Profil und Design u.ä.m. nicht unterschätzt werden.

\subsection{Organisationsstruktur}

Die Organisationsstruktur der hier vorgeschlagenen Beratungs-, Betreuungsund Vermittlungsagentur muß zwei Anforderungskriterien erfüllen: Sie muß zum einen ein landesweit einheitliches Erscheinungsbild mit hohem Erkennungswert gewährleisten und sie muß zum anderen eventuell bereits vorhandene Institutionen bzw. Kooperationspartner im staatlichen wie im privaten Bereich vor Ort flexibel integrieren können. 
Um dies zu gewährleisten, sollte als erstes bei der Landesregierung eine Dachorganisation gegründet werden, bei der folgende Aufgaben angesiedelt wären:

- Entwurf einer einheitlichen, wenn auch flexiblen Arbeitsbeschreibung für die Agenturen vor Ort, entsprechend der im vorhergehenden Abschnitt skizzierten Aufgaben.

- Akquise- und Marketingtätigkeiten gegenüber möglichen überregionalen Arbeitgebern sowie gegenüber potentiellen überregionalen Geldgebern wie beispielsweise der Europäischen Union.

- Installierung einer einheitlichen Datenerhebung zur wissenschaftlichen Begleitforschung und Evaluation der Erfolgsträchtigkeit der Agenturen. Dies ist verbunden mit der Installierung einheitlicher EDV-Hardware und -Software, denn eine umfassende Datenerhebung mit Hilfe der EDV kann den Vermittlungsprozeß in den Feldern der Stellenakquise, des Controlling und der fiskalischen und sozialen Kosten-Nutzen-Rechnung unterstützen. D.h. der Einsatz von EDV ist nicht nur zur Ex-post-Analyse, sondern auch und gerade zur erfolgreichen Betreibung der Agenturen notwendig. Voraussetzung hierfür ist eine geeignete Software, die im notwendigen Umfang allerdings noch nicht vorhanden ist.

- Vernetzung der dezentralen Agenturen vor Ort untereinander und mit der Dachorganisation zur Erhöhung der Kooperation und damit letztendlich der Effektivität.

Jeder Kreis bzw. jede kreisfreie Stadt ist dann verpflichtet, entsprechend der Vorgaben der Dachorganisation innerhalb eines halben Jahres eine lokale Beratungs-, Betreuungs- und Vermittlungsagentur zu realisieren. Die konkrete Form dieser Agenturen kann und soll an dieser Stelle allerdings nicht fixiert werden, da sie von den Gegebenheiten vor Ort abhängig ist. So können beispielsweise bereits Beschäftigungsinitiativen existieren, die in das aufzubauende Netzwerk integriert werden sollten. Daneben kann in jeder Stadt bzw. jedem Kreis eine unterschiedliche Zusammensetzung an potentiellen Trägern, 
wie etwa Sozialamt, Arbeitsamt, Zeitarbeitsfirmen, private Arbeitsvermittlungen, mit jeweils unterschiedlichem Potential und Interesse vorhanden sein. Hier sollte die Vielfalt und die damit verbundene schöpferische Kraft nicht beschnitten werden. Zudem erscheint ein Beirat, bestehend aus Vertretern der örtlichen Institutionen, zur Unterstützung der Agenturarbeit sinnvoll. Dies dürfte auch deswegen notwendig sein, da der Aufbau von Reputation auch Zeit braucht, die durch einen solchen Beirat verkürzt werden könnte.

Am Ende dieser Initiierungsphase, die vom Start der Dachorganisation bis zum Arbeitsbeginn der lokalen Agentur nicht länger als ein Jahr dauern sollte, sollte dann in jeder Stadt bzw. jedem Kreis eine privatrechtlich organisierte Agentur existieren, die die jeweiligen arbeitsfähigen Arbeitslosenhilfe- und Sozialhilfeempfänger berät, betreut und vermittelt.

Die Arbeit der Agentur sollte schließlich durch finanzielle Anreize gefördert werden. Vorstellbar ist, daß die Agentur $50 \%$ der eingesparten Arbeitslosenoder Sozialhilfe bekommt, wenn die betreffende Person sechs Monate nach der Vermittlung immer noch in Beschäftigung ist. Ein weiterer Bonus könnte fällig werden, wenn der ehemalige Maßnahmenteilnehmer nach drei Jahren immer noch beschäftigt ist. Zusätzlich sollten diese Prämien vom Ausmaß des Arbeitsvolumens abhängig sein, da ein ehemaliger Leistungsbezieher in einer Vollzeitbeschäftigung mehr Mittel für die jeweilige Transferinstitution einspart als in einer Teilzeitbeschäftigung, die zu einem weiteren, wenn auch geringeren Transferbezug berechtigt. Analog zu diesen Anreizen sollten auch für die Agenturmitarbeiter erfolgsabhängige Entgeltstrukturen vorgesehen werden.

Die Kosten dieser Agenturen sind nicht von vornherein zu quantifizieren, da sie vor allem von den institutionellen wie personellen Strukturen und Ausstattungen der für eine solche Einrichtung möglichen Träger vor Ort abhängig sind. Die hier vorgeschlagene Reorganisation der Arbeitsmarktpolitik sollte jedoch freie Kapazitäten in den Transferinstitutionen, aber auch in den anderen erwähnten Organisationen schaffen, so daß die Kosten in diesem Bereich als relativ gering zu veranschlagen sind. Gleiches gilt für die Dachorganisation. Der zweite Bestimmungsfaktor für die Kosten ist in der Betreuungsrelation zu sehen. Diese liegt beispielsweise im Projekt 1000 Ressourcen bei 1:10. im britischen 
New Deal-Programm bei 1:60 und bei den Arbeitsämtern bei etwa 1:700. Will man den Erfolg der Agenturen nicht durch eine zu geringe Personalausstattung gefährden, sollte mittelfristig eine Betreuungsrelation von 1:20 angestrebt werden - dies indizieren zumindest die Analysen aus Kapitel 3.2. An dieser Stelle sei nochmals darauf verwiesen, daß die Lohnsubventionen, so wie sie hier vorgeschlagen wurden, nicht zu Mehrkosten führen, sondern im ungünstigsten Fall kostenneutral sind, eher aber Ersparnisse bei der Sozial- und Arbeitslosenhilfe generieren.

Die Beratungs-, Betreuungs- und Vermittlungsagenturen stellen somit ein sinnvolles und notwendiges, aber auch prinzipiell finanzierbares Komplement zu den Lohnsubventionen dar. 


\section{Potentialabschätzung}

Zur Abschätzung der maximalen Anzahl zusätzlicher Beschäftigungsverhältnisse durch das Instrument der Lohnsubvention ist sowohl die Arbeitsangebots- als auch die Arbeitsnachfrageseite bezogen auf Hessen zu betrachten.

Auf der Arbeitsangebotsseite wird zunächst der quantitative Umfang der Zielgruppe von Lohnsubventionen bestimmt. Nachfrageseitig werden die Arbeitsbereiche betrachtet, in denen Arbeitsplätze geschaffen werden könnten. Die Anzahl dieser vornehmlich im Niedriglohnbereich und hierbei insbesondere im primären Dienstleistungssektor angesiedelten potentiellen Beschäftigungsverhältnisse wird ebenfalls abgeschätzt. Das Potential der mit Hilfe der Lohnsubvention erreichbaren zusätzlichen Beschäftigung läßt sich dann durch einen Vergleich von Angebot und Nachfrage beziffern. Das tatsächliche Arbeitsangebotsverhalten der Zielgruppenmitglieder kann im Rahmen dieser Studie allerdings nicht berücksichtigt werden, da dies eine umfangreiche Erhebung von Arbeitsangebotselastizitäten erfordern würde.

\subsection{Arbeitsangebot}

Zielgruppe der Lohnsubventionen sind langzeitarbeitslose Empfänger von Sozialhilfe und/oder Arbeitslosenhilfe in Hessen, d.h. solche Personen, die seit mindestens einem Jahr arbeitslos gemeldet und Empfänger von laufender Hilfe zum Lebensunterhalt oder von Leistungen nach dem Arbeitsförderungsgesetz (AFG) sind. Rechtlich soll der Bezug von Lohnsubventionen also am Bezug von Sozialhilfe oder Arbeitslosenhilfe und der Dauer der registrierten Arbeitslosigkeit festgemacht werden, d.h. wer langzeitarbeitslos und bedürttig im Sinne des BSHG ist, hat bei niedrig entlohnter Arbeit einen Anspruch auf Lohnsubvention.

Grundlage der Berechnungen des Arbeitsangotspotentials ist die jährliche Bestandserhebung zum 31. Dezember der Empfänger laufender Hilfe zum Lebensunterhalt (HLU) für das Jahr 1997 (Hessisches Statistisches Landesamt i.E.). Von den 1997 arbeitslos gemeldeten Sozialhilfeempfängern (rd. 61.000) 
- von denen man annehmen kann, daß sie mittelfristig dem Arbeitsmarkt zur Verfügung stehen, da sie nicht aus anderen Gründen (z.B. Krankheit etc., siehe unten) Sozialhilfe beziehen - waren rd. 40.000 Personen länger als ein $1 \mathrm{Jahr}$ arbeitslos und gehören somit zur Zielgruppe der Lohnsubventionen.

Hinzugezählt werden müssen die Arbeitslosenhilfeempfänger, da auch sie mindestens ein Jahr arbeitslos sind und somit der Zielgruppe angehören. Allerdings ist zu beachten, daß es hier Überschneidungen zwischen der Gruppe der langzeitarbeitslosen Sozialhilfeempfänger und der Empfänger von Arbeitslosenhilfe gibt. Zur Vermeidung von Doppelzählungen müssen diejenigen Personen herausgerechnet werden, die zur Arbeitslosenhilfe ergänzende Sozialhilfe erhalten. Da die personenbezogene Sozialhilfestatistik der Empfänger von laufender Hilfe zum Lebensunterhalt keine Unterscheidung zwischen Arbeitslosenhilfe und -geld macht, muß an dieser Stelle eine Abschätzung mit Hilfe der Daten zur Dauer der Arbeitslosigkeit von Empfängern von HLU mit Leistungen nach AFG getroffen werden. Von den Ende 1997 erfaßten 22.502 Empfängern von Hilfe zum Lebensunterhalt und Leistungen nach AFG waren 15.035 Personen länger als 1 Jahr arbeitslos, so daß man vermuten kann, daß diese auch Empfänger von Arbeitslosenhilfe waren.

Allerdings ist diese Schätzung der Doppelzählung vermutlich zu hoch angesetzt, denn erfahrungsgemäß beziehen nicht alle in der Statistik der Sozialhilfe als Bezieher von AFG-Leistungen aufgeführten Personen tatsächlich diese Leistungen. So ergab eine Untersuchung in der Stadt Wiesbaden (Brennecke 1996, 47f), daß von 1.454 18-60jährigen HLU-Beziehern, deren "Merker" auf „arbeitslos mit AFG-Leistungen“ gesetzt waren, nur knapp 70\% tatsächlich Arbeitslosengeld oder -hilfe bezogen haben. In den übrigen Fällen war entweder

- der Anspruch auf AFG-Leistungen zwischenzeitlich ausgelaufen,

- hatten die entsprechenden Hilfeempfänger mittlerweile eine Erwerbstätigkeit aufgenomen,

- nahmen an einer Umschulungsmaßnahme teil oder

- waren enwerbsunfähig.

Erstere dürfen dann nicht als Doppelzählung vom Potential abgezogen werden, da sie gar keine Leistungen mehr nach AFG erhalten, und somit auch nicht in 
der Statistik der Arbeitslosenhilfeempfänger erscheinen. Nur die übrigen drei Gruppen müssen - auch wenn sie keine Doppelzählungen darstellen - vom Potential abgezogen werden, da sie aus anderen Gründen dem Arbeitsmarkt nicht zur Verfügung stehen. Somit ist das errechnete Potential der nicht Arbeitslosenhilfe empfangenden Personen - quantitativ unbestimmbar - höher anzusetzen, als es die folgende Tabelle wiedergibt.

Nach Hinzurechnung der Bezieher von Arbeitslosenhilfe (rund 70.000) ergibt sich somit ein gesamtes mit Hilfe von Lohnsubventionen erreichbares Arbeitsangebotspotential von knapp 95.000 Personen.

\begin{tabular}{l|l|r}
\hline & \multicolumn{1}{|c|}{1997} \\
\hline$(1)$ & Empfänger laufender Hilfe zum Lebensunterhalt (nachrichtlich) & 262.196 \\
$(2)$ & davon zwischen 15 und 65 Jahren, länger als 1 Jahr arbeitslos ge- & 39.994 \\
& meldet und erwerbsfähig & \\
(3) & - Doppelzählungen (Empfänger von Arbeitslosenhilfe, geschätzt) & 15.035 \\
\hline & $=$ & 24.959 \\
$(4)$ & + Bezieher von Arbeitslosenhilfe & 69.947 \\
\hline & $=$ & 94.906 \\
& zotential des Arbeitsangebots einer Lohnsubvention an lang- & \\
\hline
\end{tabular}

Tabelle 4: Zielgruppenbestimmung einer Lohnsubvention an Langzeitarbeitslose in Hes$\operatorname{sen}^{33}$

Dieses Potential ist aus zwei Gründen als untere Grenze anzusehen: Zum einen lassen sich in diesem Potential aufgrund der statistischen Daten nur solche Sozialhilfeempfänger einrechnen, die auch tatsächlich arbeitslos gemeldet sind. Werden Lohnsubventionen an Langzeitarbeitslose gewährt, so ist anzunehmen, daß sich arbeitsfähige, aber nicht arbeitende Personen arbeitslos melden werden, um nach einem Jahr registrierter Arbeitslosigkeit eine subventionierte Arbeit annehmen zu können.

Zum anderen könnte die Subvention zur Vermeidung weiterer Humankapitalverluste an alle Sozialhilfeempfänger geleistet werden.

${ }^{33}$ Berechnungen nach Hessisches Statistisches Landesamt (i.E.): Tabellen E7.1, E7.2, (4) Landesarbeitsamt Hessen, Durchschnitt für 1997. 
Somit können neben den Langzeitarbeitslosen auch solche Personen erfaßt und aus der Sozialhilfe geführt werden, die erst kurze Zeit in der Sozialhilfe verweilen.

Das dann zusätzlich erreichbare Arbeitsangebotspotential errechnet sich wie folgt:

1997 befanden sich von den registrierten 262.196 Empfängern laufender Hilfe zum Lebensunterhalt rund 161.000 im erwerbsfähigen Alter zwischen 15 und 65 Jahren, wovon etwa 12.500 bereits einer Teil- oder Vollzeitbeschäftigung nachgingen. Während die Vollzeitbeschäftigten nicht dem Potential zugerechnet werden können, kann man bei den Teilzeitbeschäftigten davon ausgehen, daß sie in der Mehrheit einer geringfügigen Beschäftigung nachgehen und vermutlich aufgrund der Anreizstrukturen der Sozialhilfe sogar nur einer (offiziellen) Beschäftigung, die mit maximal dem anrechnungsfreien Betrag von 135 DM entlohnt wird. Im Falle einer Lohnsubvention bestünde also auch für diese Gruppe ein Anreiz, das Arbeitsangebot auszuweiten, weshalb sie zum Potential hinzuzufügen ist.

Von den Nichterwerbstätigen müssen diejenigen herausgerechnet werden, die aufgrund von Alter, Krankheit, Behinderung, Arbeitsunfähigkeit, Kindererziehung, ausländerrechtlichem Status (Bürgerkriegsflüchtlinge ${ }^{34}$ ) oder arbeitsmarktpolitischer Maßnahmen dem Arbeitsmarkt nicht zu Verfügung stehen (vgl. Klopfleisch/Sesselmeier/Setzer 1997a, 233).

Nach Abzug dieser nicht-arbeitsfähigen Personen ergibt sich ein Arbeitsangebotspotential der HLU-Empfänger von 102.832 .

34 Bei der Gruppe der Bürgerkriegsflüchtlinge kann man davon ausgehen, daß sie aufgrund des nur befristeten Aufenthalts und der Sprachschwierigkeiten keine Arbeit annehmen werden. Demgegenüber besitzen Asylberechtigte eine unbefristete Aufenthaltserlaubnis, die i.d.R. auch mit einer Arbeitserlaubnis verknüpft ist. 


\begin{tabular}{|c|c|c|}
\hline & & 1997 \\
\hline (1) & Empfänger laufender Hilfe zum Lebensunterhalt & 262.196 \\
\hline \multirow[t]{2}{*}{ (2) } & - jünger als 15 und älter als 65 Jahre & 100.869 \\
\hline & $\begin{array}{l}\text { = verbleibende Personen } \\
\text { - davon enwerbstätig (vollzeit) }\end{array}$ & $\begin{array}{r}161.327 \\
5.875\end{array}$ \\
\hline $\begin{array}{l}(4) \\
(5) \\
(6) \\
(7) \\
(8)\end{array}$ & $\begin{array}{l}\text { = Nicht oder teilzeiterwerbstätige Empfänger laufender Hilfe zum } \\
\text { Lebensunterhalt zwischen } 15 \text { und } 65 \text { Jahren } \\
\text { - wegen Aus- und Fortbildung } \\
\text { - wegen häuslicher Bindung } \\
\text { - wegen Krankheit, Behinderung, Arbeitsunfähigkeit } \\
\text { - aus Altersgründen } \\
\text { - Bürgerkriegsflüchtlinge }\end{array}$ & $\begin{array}{r}155.452 \\
10.173 \\
26.261 \\
12.096 \\
2.404 \\
1668\end{array}$ \\
\hline (8) & $\begin{array}{l}=\text { Empfänger laufender Hilfe zum Lebensunterhalt zwischen } 15 \text { und } \\
65 \text { Jahren, teilzeiterwerbstätig oder nicht erwerbstätig aus sonsti- } \\
\text { gen Gründen } \\
\text { - Doppelzählungen (Empfänger von Arbeitslosenhilfe, geschätzt) }\end{array}$ & 15.035 \\
\hline \multirow[t]{2}{*}{ (10) } & $\begin{array}{l}= \\
+ \text { Bezieher von Arbeitslosenhilfe }\end{array}$ & $\begin{array}{l}87.797 \\
69.947\end{array}$ \\
\hline & $\begin{array}{l}=\text { Potential des Arbeitsangebots einer Lohnsubvention an Emp- } \\
\text { fänger von laufender Hilfe zum Lebensunterhalt und/oder Ar- } \\
\text { beitslosenhilfe }\end{array}$ & 157.744 \\
\hline
\end{tabular}

Tabelle 5: Zielgruppenbestimmung einer Lohnsubvention an alle Arbeitslosenhilfe- und Sozialhilfeempfänger in Hessen ${ }^{35}$

Von diesen müssen wieder zur Vermeidung von Doppelzählungen diejenigen Personen herausgerechnet werden, die Sozialhilfe ergänzend zur Arbeitslosenhilfe erhalten. Hierbei gelten die Abschätzungen und Annahmen der ersten Potentialberechnung.

Hinzu kommen auch hier die rund 70.000 Arbeitslosenhilfeempfänger, so daß sich für Hessen ein zusätzliches durch Lohnsubventionen an alle Sozialhilfeund Arbeitslosenhilfeempfänger aktivierbares Arbeitsangebotspotential von rund 158.000 Personen ergibt.

Die Dynamik in diesen Potentialen wird bei einem Vergleich der Zahlen von 1997 mit denen für 1996 deutlich. Vor allem aufgrund des starken Anstiegs der

${ }^{35}$ Berechnungen nach Hessisches Statistisches Landesamt (i.E.): Tabellen E1.1, E2, E6.1, E7.1, E7.2, B10.1; (10) Landesarbeitsamt Hessen, Durchschnitt für 1997. 
Anzahl von Empfängern von Arbeitslosenhilfe wuchsen beide Potentiale gegenüber 1996 um über 15.000 Personen $^{36}$. Damit wird auch in Hessen der bundesweite Trend stagnierender bzw. nicht sinkender Arbeitslosigkeit bei gleichzeitig ansteigender Langzeitarbeitslosigkeit sichtbar.

Je nach Zielgruppenabgrenzung wäre somit in Hessen ein Angebotspotential von 95.000 bis 158.000 Personen aktivierbar.

\subsection{Arbeitsnachfrage}

Während bei der Bestimmung der Zielgruppe einer Lohnsubvention danach gefragt wurde, wer aufgrund seiner grundsätzlich vorhandenen Arbeitsfähigkeit bei einem zusätzlichen Anreiz Arbeit anbieten könnte, ist bei der Abschätzung des Nachfragepotentials zunächst zu fragen, in welchen Bereichen die Arbeit des Angebotspotentials nachgefragt werden könnte, um diese Nachfrage anschließend zu quantifizieren. Der Markt sollte hierbei als Entdeckungsverfahren dienen, d.h. wenn die Zielgruppe Arbeit preiswerter anbieten kann als bisher, sollte der Markt in der Lage sein, Angebot und Nachfrage zu neuen Beschäftigungsverhältnissen zusammenzuführen. Dahinter steht die Idee, daß Lohnsubventionen den Rückstand Deutschlands gegenüber anderen europäischen Staaten im niedrig entlohnten Dienstleistungsbereich aufholen könnten, indem sozialpolitisch manifestierte Mindestlöhne und die oben beschriebenen Anreizprobleme aufgehoben werden.

\subsubsection{Allgemeine Vorgehensweise}

Zur Bestimmung der Arbeitsnachfrage müssen zunächst Branchen oder Arbeitsfelder identifiziert werden, in denen die Zielgruppe der Lohnsubventionen Arbeit finden könnte. Erste Hinweise ergeben sich bei Betrachtung des Qualifikationsgrades der Personen dieser Gruppe. Insbesondere bei den langzeitarbeitslosen Sozialhilfeempfängern handelt es sich in der Mehrzahl um Personen mit niedriger Qualifikation (vgl. Leibfried/Leisering u.a. 1995, 100). Betrachtet

${ }^{36}$ Berechnungen nach Hessisches Statistisches Landesamt 1998. 
man sich die durchschnittliche Dauer der Hilfegewährung in Abhängigkeit von Schul- und Berufsabschluß im Jahr 1996 (vgl. Hessisches Statistisches Landesamt 1998, Tabelle E11.1), so fällt auf, daß sie bei den Sozialhilfebeziehern ohne oder mit unbekanntem Schulabschluß mit 19,8 bzw. 19,2 Monaten sowie ohne oder mit unbekanntem beruflichen Ausbildungsabschluß mit 17 bzw. 19,6 Monaten deutlich über der durchschnittlichen Dauer der übrigen Gruppen liegen (Fachhochschul-/Hochschulreife 12,5 Monate; abgeschlossene beruflichschulische Ausbildung 12,7 Monate).

Eine Beschäftigung dieser Zielgruppe wird also vorwiegend in solchen Bereichen möglich sein, in denen geringe Qualifikationsanforderungen des Arbeitsangebots mit einer geringen Zahlungsbereitschaft bzw. hoher Preiselastizität der Arbeitsnachfrage zusammenfallen.

Eine Abschätzung der Nachfrage eines solchen "low-wage-Segments" erfolgt mit Hilfe eines Vergleichs von Beschäftigungsdichten ausgewählter europäischer Staaten und Deutschlands, die anschließend mit dem Bevölkerungsanteil Hessens gewichtet werden. Die Beschäftigungsdichten werden dabei für Tätigkeitsfelder (ausgeübte Tätigkeiten) berechnet. Alternativ könnten Beschäftigungsdichten für Branchen berechnet und verglichen werden ${ }^{37}$, allerdings treten hier Probleme bei unterschiedlichem Niveau der vertikalen Integration in den betrachteten Staaten auf. Länder, in denen die Auslagerung von produktionsbezogenen Dienstleistungen schon weit fortgeschritten ist, weisen dann eine hohe Beschäftigung in den entsprechenden Dienstleistungsbranchen auf, obwohl die gleichen Stellen und damit Qualifikations- und Entlohnungsbereiche in Volkswirtschaften mit einem höheren Grad der vertikalen Integration in den Branchen des sekundären Sektors besetzt sein könnten (vgl. Cornetz, W./Schäfer, H. 1998, 419).

37 Diese Vorgehensweise findet sich u.a. bei der Zukunftskommission der Friedrich-EbertStiftung 1998, $235 f$. 


\subsubsection{Datengrundlage}

Das Ziel eines Vergleichs der Beschäftigungsdichten sollte eigentlich sein, einen Zusammenhang zwischen ausgeübten Tätigkeiten und erzieltem Einkommen bzw. der Lohnhöhe oder Qualifikationsstufen am Arbeitsplatz herzustellen, um die relevanten Bereiche herauszufiltern und anschließend ein Potential für Deutschland zu berechnen. Allerdings sind solche Kreuzdaten weitestgehend nicht verfügbar. Die New Chronos Datenbank von EUROSTAT enthält zwar derartige kreuztabellierte Daten, diese sind zur Zeit aber noch zu lückenhaft, um darauf eine valide Potentialabschätzung aufbauen zu können. Auch die von der ILO verfügbaren Daten (Statistics on occupational wages and hours of work) erfassen international nur durchschnittliche Stunden-, Wochen- und Jahresverdienste auf hochaggregierter Ebene, so daß auch diese Daten keine Gruppierung der Tätigkeitsfelder nach Löhnen ermöglichen.

Aus diesen Gründen werden auf der Basis von Plausibilitätsüberlegungen Tätigkeitsfelder identifiziert, die Geringqualifizierten bei entsprechender Entlohnung zusätzliche Arbeitsplätze bieten könnten.

Datengrundlage ist die von der EU jährlich durchgeführte und nach ISCO-88 klassifizierte Arbeitskräfteerhebung (Labour Force Survey).

Die wesentlichen Vorteile dieser Arbeitskräfteerhebung sind:

- Die einheitliche Erhebung der Daten durch die nationalen statistischen Ämter gewährleistet eine internationale Vergleichbarkeit.

- Der jährliche Erhebungszyklus gewährleistet die Aktualität der Daten.

- Die Datensätze sind weitgehend vollständig.

- Das verwendete Klassifikationsschema (ISCO-88) erlaubt eine detaillierte Aufgliederung nach Berufen.

Nachteilig an der verwendeten Arbeitskräfteerhebung ist die Beschränkung auf die Mitgliedsstaaten der EU. Insbesondere ein Vergleich mit den USA ist dadurch nicht möglich ${ }^{38}$. Darüber hinaus wurde das verwendete Klassifikations-

38 Eine Potentialabschätzung auf Grundlage von tätigkeitsbezogenen Beschäftigungsdichten in den USA enthält Sesselmeier/Klopfleisch/Setzer 1996, 153ff. Das zusätzliche Arbeitsnachfragepotential für Deutschland errechnet sich dort zu 2,75 Mio. Personen. Für 
schema im Jahr 1991 grundlegend verändert, so daß die hier nutzbaren Datensätze "nur" den Zeitraum 1992 bis 1997 umfassen. Da die Berechnung des Beschäftigungspotentials aber zeitpunktbezogen erfolgt (s.u.), kommt dieser Nachteil hier nicht zum Tragen.

\subsubsection{Berechnungsmethodik}

Die Berechnung des Beschäftigungspotentials kann prinzipiell zeitraum- oder zeitpunktbezogen erfolgen. Eine zeitraumbezogene Berechnung kann die Wachstumsdynamik in verschiedenen Bereichen aufzeigen. Aufgrund der kurzen Zeitreihen der vorhandenen Daten wird diese Methode aber hier nicht weiter verfolgt, sondern es wird eine zeitpunktbezogene Berechnung des Beschäftigungspotentials mit dem Bezugsjahr 1997 durchgeführt.

Dabei wird zunächst für jede Berufsgruppe der dreistelligen ISCO-88 Klassifizierung die 1997 erreichte Beschäftigungsdichte im Vergleichsland berechnet, indem die aktuelle Beschäftigung auf die Bevölkerung bezogen wird. Diese Dichte multipliziert mit der Bevölkerung in Deutschland ergibt die Anzahl der hier c.p. möglichen Beschäftigungsverhältnisse in den entsprechenden Bereichen. Davon wird die Anzahl der schon vorhandenen Beschäftigungsverhältnisse in Deutschland abgezogen, so daß sich als Differenz das zusätzlich mögliche Beschäftigungspotential ergibt ${ }^{39}$.

Die Berechnung erfolgt für jede relevante Berufsgruppe des Klassifikationsschemas nach folgender Formel:

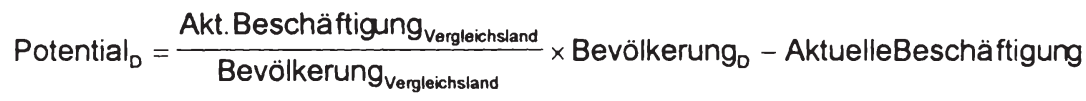

Als Vergleichsländer wurden Dänemark, die Niederlande und Großbritannien gewählt, da gerade diese Länder innerhalb Europas eine bessere Arbeitsmarktperfomance aufweisen als Deutschland und diese Staaten schon früh

\footnotetext{
Hessen, gewichtet mit der Bevölkerungsanzahl, bedeutet dies ein Potential von 206.000 Personen.

39 Die gleiche Vorgehensweise - allerdings auf anderer Datengrundlage - findet sich bei Klös 1997a.
} 
damit begonnen haben, Programme für niedrig entlohnte Arbeitsplätze zu entwickeln. Einem derartigen Vergleich liegt natürlich die zunächst rigide Annahme gleicher Lohn- und Nachfragestrukturen in den Vergleichsländern zugrunde. Auch wenn diese Vorgehensweise als erste Annäherung sinnvoll ist, müssen doch einige, die Ergebnisse relativierende Ergänzungen angebracht werden. Die Einschränkungen resultieren insbesondere aus unterschiedlichen institutionellen und sozio-kulturellen Faktoren. So werden unter Umständen für manche der hier ermittelten Arbeitsplätze im Niedriglohnsektor wie etwa im Bereich der Kinderbetreuung in Deutschland wesentlich höhere Qualifikationen als in den Vergleichsländern verlangt. Daneben beruht die hier konstatierte Dienstleistungslücke auch auf einer mangelnden Nachfrage dieser Serviceleistungen aufgrund spezifischer traditioneller Handlungsweisen. Gleichwohl sind diese Faktoren nicht als unveränderbare und unveränderliche Konstanten zu betrachten, so daß die hier vorgeführte Vorgehensweise als vertretbar erscheint (vgl. Klopfleisch/Sesselmeier/Setzer 1997a).

\subsubsection{Auswahl der relevanten Berufsgruppen}

Der ISCO-88 Index unterscheidet auf der obersten Ebene neun Berufsgruppen (vgl. Tabelle 6) innerhalb derer mindestens zwei Gruppen identifiziert werden können, von denen man ein unterdurchschnittliches Qualifikationsniveau erwarten kann, nämlich die Sparten Hilfsarbeitskräfte (9) und Dienstleistungsberufe (5).

\begin{tabular}{|ll|}
\hline 1 & Legislators, senior officials and managers \\
2 & Professionals \\
3 & Technicians and associate professionals \\
4 & Clerks \\
5 & Service workers and shop and market sales workers \\
6 & Skilled agricultural and fishery workers \\
7 & Craft and related trade workers \\
8 & Plant and machine operators and assemblers \\
9 & Elementary occupations \\
\hline
\end{tabular}

Tabelle 6: ISCO-88 Index

Ebenfalls relevant erscheinen Teilbereiche der Gruppe 4 (Bürokräfte und kaufmännische Angestellte). Insgesamt kann diese Gruppe allerdings nicht zur 
Potentialberechnung herangezogen werden, da hier auch qualifizierte Personen tätig sind. Relevant in dieser Gruppe erscheinen die Untergruppen

- Material recording and transport clerks,

- Library, mail and related clerks,

- Other office clerks,

- Cashiers, tellers and related clerks sowie

- Client information clerks.

In Gruppe 5 lassen sich für eine zusätzliche Bestimmung eines unteren Potentials besonders relevante Untergruppen identifizieren, die einen größeren Anteil an unqualifizierten Beschäftigten aufweisen als die gesamte Gruppe. Dies sind

- Travel attendants and related workers,

- Housekeeping and restaurant services workers,

- Personal care and related workers,

- Other personal service workers,

- Protective services workers,

- Shop salespersons and demonstrators.

In Gruppe 9 erscheinen dagegen alle Untergruppen relevant:

- Street vendors and related workers,

- Shoe cleaning and other street services elementary occupations,

- Domestic and related helpers, cleaners and launderers,

- Building caretakers, window and related cleaners,

- Messengers, porters, doorkeepers and related workers,

- Garbage collectors and related labourers,

- Agricultural, fishery and related labourers,

- Mining and construction labourers,

- Manufacturing labourers,

- Transport labourers and freight handlers.

Zudem erscheinen aus den Gruppen 7 und 8 folgende Kategorien relevant:

- Painters, building structure cleaners and related trades workers, 
- Food processing and related trades workers,

- Assemblers sowie

- Motor vehicle drivers.

Bei der folgenden Potentialabschätzung muß beachtet werden, daß die durchschnittlichen Arbeitszeiten in den untersuchten Staaten teilweise voneinander abweichen, so daß ein Arbeitszeitfaktor mitberücksichtigt wird. Anschließend wird ein Potential für Hessen auf der Grundlage des hessischen Bevölkerungsanteils an der Gesamtbevölkerung Deutschland berechnet. Dies ist natürlich eine vereinfachende Vorgehensweise, da die Strukturen, wie schon beim bilateralen Vergleich auch nicht zwischen Deutschland und einem Bundesland 1:1 übertragbar sind. In Ermangelung einer international vergleichbaren Statistik dieser Daten für Hessen erscheint diese Vorgehensweise aber dennoch als vertretbar, mit einem einschränkenden Hinweis. Denn aufgrund der hohen Kaufkraft innerhalb des Rhein-Main-Gebietes und der damit vermutlich großen Differenz zwischen eigenem Nettolohn und dem Preis der dann angebotenen Dienstleistungen könnten die hier zu treffenden make or buy Entscheidungen dann vornehmlich zugunsten "buy" ausfallen, so daß das tatsächliche Potential der Arbeitsnachfrage vermutlich höher als das errechnete ausfällt.

\subsubsection{Potentialberechnung}

Die Berechnung der beiden Potentiale für Deutschland ist in den folgenden Übersichten dargestellt. Potential 1 berücksichtigt - als eine erste grobe Schätzung - die Hauptgruppen 5 und 9, sowie ausgewählte Untergruppen aus 4, wobei hierbei teilweise Untergruppen mitberücksichtigt werden, in denen nicht durchgängig ein unterdurchschnittliches Qualifikationsniveau zu erwarten ist. Potential 2 berücksichtigt dagegen nur die ausgewählten Untergruppen, wobei bei dieser engen Auswahl im Zweifel Untergruppen unberücksichtigt blieben, obwohl sie teilweise relevant erschienen. Die Potentiale entspringen demnach unterschiedlichen Methoden der Abschätzung, wobei die Ergebnisse aber durchaus weniger voneinander abweichen, als zunächst zu erwarten ist. 
Die Gewichtung der Potentiale mit den durchschnittlichen Arbeitszeiten innerhalb der Gruppen verändert das Ergebnis nur beim Vergleich mit den Niederlanden, da die Arbeitszeiten dort vor allem in den Hauptgruppen 5 und 9 (24,5 und 23,8 Wochenstunden) deutlich niedriger liegen als in Deutschland $(33,5$ und 32,8$)$. Die Unterschiede gegenüber Großbritannien $(28,2$ und 29,8$)$ und Dänemark $(30,6$ und 26,7$)$ fallen dagegen geringer aus (eurostat 1995, table 075).

\begin{tabular}{|c|c|c|c|c|}
\hline \multirow{2}{*}{$\begin{array}{l}\begin{array}{l}\text { Haupt- } \\
\text { gruppen }\end{array} \\
\text { Clerks }\end{array}$} & \multirow[t]{2}{*}{$\begin{array}{l}\text { ausgewählte } \\
\text { Untergruppen }\end{array}$} & D-DK & \multirow[t]{2}{*}{$\begin{array}{l}\text { Potential } 1 \\
\text { D-NL }\end{array}$} & D-UK \\
\hline & & & & \\
\hline & $\begin{array}{l}\text { Material recording and trans- } \\
\text { port clerks }\end{array}$ & 241.332 & 272.870 & -31.174 \\
\hline & Library, mail and related clerks & 196.838 & 198.473 & 441.970 \\
\hline & Other office clerks & 200.852 & -996.174 & -264.350 \\
\hline & $\begin{array}{l}\text { Cashiers, tellers and related } \\
\text { clerks }\end{array}$ & 82.338 & 251.944 & 575.830 \\
\hline & Client information clerks. & 207.798 & 299.826 & 228.995 \\
\hline \multicolumn{2}{|c|}{$\begin{array}{l}\text { Service workers and shop and Market } \\
\text { sales workers }\end{array}$} & 2.374 .347 & 690.250 & 1.513 .544 \\
\hline \multicolumn{2}{|c|}{ Elementary occupation } & 2.048.396 & 18.335 & 378.759 \\
\hline \multicolumn{2}{|l|}{ Summe } & 5.351 .901 & 735.524 & 2.843.575 \\
\hline \multicolumn{2}{|c|}{ gewichtet mit Arbeitszeit } & 4.727.813 & 541.472 & 2.544 .732 \\
\hline \multicolumn{2}{|c|}{ Potential für Hessen } & 353.969 & 40.540 & 190.515 \\
\hline
\end{tabular}

Tabelle 7: Arbeitsnachfragepotentiale einer Lohnsubvention für Deutschland (Potential 1) 


\begin{tabular}{|c|c|c|c|c|}
\hline $\begin{array}{l}\text { Haupt- } \\
\text { gruppen }\end{array}$ & $\begin{array}{l}\text { ausgewählte } \\
\text { Untergruppen }\end{array}$ & D-DK & $\begin{array}{l}\text { Potential } 2 \\
\text { D-NL }\end{array}$ & D-UK \\
\hline \multicolumn{5}{|l|}{ Clerks } \\
\hline & $\begin{array}{l}\text { Material recording and trans- } \\
\text { port clerks }\end{array}$ & 241.332 & 272.870 & -31.174 \\
\hline & Library, mail and related clerks & 196.838 & 198.473 & 441.970 \\
\hline & Other office clerks & 200.852 & -996.174 & -264.350 \\
\hline & $\begin{array}{l}\text { Cashiers, tellers and related } \\
\text { clerks }\end{array}$ & 82.338 & 251.944 & 575.830 \\
\hline & Client information clerks. & 207.798 & 299.826 & 228.995 \\
\hline \multicolumn{5}{|c|}{$\begin{array}{l}\text { Service workers and shop and Market } \\
\text { sales workers }\end{array}$} \\
\hline & $\begin{array}{l}\text { Travel attendants and related } \\
\text { workers }\end{array}$ & 29.583 & 27.173 & 53.658 \\
\hline & $\begin{array}{l}\text { Housekeeping and restaurant } \\
\text { services workers }\end{array}$ & -159.835 & -3.175 & 368.462 \\
\hline & $\begin{array}{l}\text { Personal care and related } \\
\text { workers }\end{array}$ & 2.228 .375 & 423.563 & 661.489 \\
\hline & Other personal service workers & -133.178 & -87.074 & -157.812 \\
\hline & Protective services workers & -9.275 & 19.976 & 267.172 \\
\hline & $\begin{array}{l}\text { Shop salespersons and dem- } \\
\text { onstrators }\end{array}$ & 407.501 & 310.715 & 320.574 \\
\hline \multicolumn{5}{|c|}{ Craft and related trade workers } \\
\hline & $\begin{array}{l}\text { Painters, building structure } \\
\text { cleaners and related trades } \\
\text { workers }\end{array}$ & -136.357 & -203.734 & -134.899 \\
\hline & $\begin{array}{l}\text { Food processing and related } \\
\text { trades workers }\end{array}$ & 3.507 & -77.780 & -148.937 \\
\hline \multicolumn{5}{|c|}{$\begin{array}{l}\text { Plant and machine operators and as- } \\
\text { semblers }\end{array}$} \\
\hline & Assemblers & 109.737 & -16.034 & 268.441 \\
\hline & Motor vehicle drivers & 70.636 & 22.172 & 125.448 \\
\hline
\end{tabular}

Tabelle 8: Arbeitsnachfragepotentiale einer Lohnsubvention für Deutschland (Potential 2) 


\section{Elementary occupation}

\begin{tabular}{|c|c|c|c|}
\hline $\begin{array}{l}\text { Street vendors and related } \\
\text { workers }\end{array}$ & 67.974 & 221.336 & 184.127 \\
\hline $\begin{array}{l}\text { Shoe cleaning and other street } \\
\text { services elementary occupa- } \\
\text { tions }{ }^{40}\end{array}$ & - & - & - \\
\hline $\begin{array}{l}\text { Domestic and related helpers, } \\
\text { cleaners and launderers }\end{array}$ & 999.120 & 311.061 & 455.924 \\
\hline $\begin{array}{l}\text { Building caretakers, window } \\
\text { and related cleaners }\end{array}$ & 149.775 & -48.567 & -110.290 \\
\hline $\begin{array}{l}\text { Messengers, porters, door- } \\
\text { keepers and related workers }\end{array}$ & 229.502 & -144.451 & 79.785 \\
\hline $\begin{array}{l}\text { Garbage collectors and rel. } \\
\text { labourers }\end{array}$ & 8.905 & 25.086 & -3.136 \\
\hline $\begin{array}{l}\text { Agricultural, fishery and rel. } \\
\text { labourers }\end{array}$ & 84.667 & -160.156 & 11.532 \\
\hline $\begin{array}{l}\text { Mining and construction la- } \\
\text { bourers }\end{array}$ & 255.055 & -51.850 & 99.080 \\
\hline Manufacturing labourers & -80.854 & -626.780 & -180.350 \\
\hline \multirow{2}{*}{$\begin{array}{l}\text { Transport labourers and freight } \\
\text { handlers }\end{array}$} & 334.251 & 492.655 & -157.912 \\
\hline & 5.388 .250 & 461.076 & 2.953.628 \\
\hline mit Arbeitszeit & 4.765 .129 & 266.774 & 2.654 .785 \\
\hline Hes & 356.763 & 19.973 & 198.755 \\
\hline
\end{tabular}

Tabelle 8 (Fortsetzung): Arbeitsnachfragepotentiale einer Lohnsubvention für Deutschland (Potential 2)

Auffallend groß sind die Potentialunterschiede bezogen auf die jeweiligen Vergleichsländer. Während man beim Vergleich mit Dänemark eine Dienstleistungslücke für Niedrigqualifizierte in Hessen von rund 350.000 Personen errechnet und beim Vergleich mit Großbritannien von knapp 200.000, so ist das Potential gegenüber den Niederlanden mit 40.000 bzw. arbeitszeitgewichtet 20.000 deutlich niedriger. Für das niedrige Potential im Vergleich zu den Niederlande scheinen die unterschiedlichen Strategien der in den Vergleichsländern praktizierten Arbeitsmarktpolitk eine Erklärung zu bieten. Denn während in Dänemark und Großbritannien die Absenkung des Lohnniveaus gerade in

40

Keine Daten vorhanden. 
den hier betrachteten Bereichen zusätzliche Arbeitsplätze haben entstehen lassen, streuten die Erfolge der Teilzeitarbeitsplatzoffensive in den Niederlanden über alle 9 Beschäftigungsfelder.

Die hier errechneten Potentiale sollen auch nur einen ersten Hinweis geben, was unter den Sozialstaatsbedingungen innerhalb Europas machbar und mindestens möglich erscheint. Neue, innerhalb eines Servicewettbewerbs entstehende Beschäftigungsideen können in den Potentialen nur angedeutet werden.

In welchen Bereichen aber eine deutliche Dienstleistungslücke gegenüber den Vergleichsstaaten vorhanden scheint, zeigen die Einzelpotentiale in den Untergruppen. So sind z.B. Beschäftigungsverhältnisse bei den persönlichen Pflegediensten in Deutschland vor allem im Vergleich zu Dänemark - aber auch im Vergleich zu den anderen beiden Staaten - deutlich geringer etabliert. Das Potential beträgt gegenüber Dänemark etwa 2 Mio. Personen für Deutschland. Aber auch die Beschäftigungsdichten Dänemarks in anderen haushaltsnahen Diensten sind deutlich höher als in Deutschland, so daß hier ebenfalls ein Potential von fast 1 Mio. Personen möglich erscheint. Gegenüber Großbritannien beläuft sich die Anzahl von Beschäftigungen als Kassierer in Deutschland deutlich unter dem dort zu findenden Niveau. Das demnach erreichbare Potential beträgt etwa eine halbe Mio. Personen. Ein in allen drei Staaten deutlich stärker entwickelter Beschäftigungsbereich stellen Verkäufer und Vorführer dar. Das Potential für Deutschland beträgt etwa 300.000 Personen.

Relativierend zu diesen Potentialen läßt sich einwenden, daß die Erwerbsneigung in den Vergleichsstaaten von Grund auf höher ist als in Deutschland, weshalb die Potentiale zwingenderweise groß ausfallen müssen. Das relativ niedrige Potential im Vergleich zu den Niederlanden belegt aber gerade das Gegenteil, denn nur hier verteilt sich die - von Grund auf (?) - höhere Erwerbsneigung auf alle Beschäftigungsbereiche, während der Nachholbedarf Deutschlands bei der Erwerbstätigkeit vielleicht gerade in den untersuchten niedrig entlohnten Dienstleistungsbereichen zu suchen ist, wie der Vergleich mit Großbritannien und Dänemark zeigt (vgl. Andreß/Strengmann 1997). 
Bei allen Einwänden wird zumindest deutlich, daß gegenüber Dänemark und Großbritannien eine deutliche „Dienstleistungslücke“ (rund 350.000 bzw. knapp 200.000) festzustellen ist, die größer ist als das durch Lohnsubventionen zusätzlich erreichbare Arbeitsangebot (95.000 bzw. 160.000). D.h., würde man in Deutschland die gleiche Dienstleistungsdichte der ausgewählten Niedrigqualifiziertenbereiche wie in Großbritannien und Dänemark herstellen können, so würden alle arbeitsfähigen Sozialhilfe- und Arbeitslosenhilfeempfänger in Hessen durch die zusätzliche Arbeitsnachfrage in Beschäftigung gelangen können. 
Martin Setzer, Roland Klopfleisch and Werner Sesselmeier - 978-3-631-75277-7

Downloaded from PubFactory at 01/11/2019 06:22:32AM

via free access 


\section{$7 \quad$ Abschließende Bemerkungen}

Die deutsche Arbeitsmarktsituation, die sich im Laufe der letzten Jahre im negativen Sinne von der Entwicklung der Arbeitsmärkte in einer großen Zahl anderer EU- bzw. OECD-Staaten abgekoppelt hat, erfordert neue situationsgerechte und zielgruppenspezifische Maßnahmen, die auf den Ersten Arbeitsmarkt ausgerichtet sind. Lohnsubventionen erfüllen diese Anforderungen. Gleichwohl erscheinen an dieser Stelle einige einschränkende und zugleich weiterführende Hinweise notwendig.

Die Tatsache fehlender Arbeitsnachfrage im Bereich binnenmarktorientierter Dienstleistungen ist nicht nur mit nach unten inflexiblen Löhnen zu erklären, sondern auch und gerade mit spezifischen soziokulturellen Einstellungen gegenüber diesen Arbeitsmöglichkeiten. Daraus folgt, daß ein Modellversuch einen spezifischen Zeithorizont von mindestens einem Konjunkturzyklus benötigt sowie begleitende Unterstützungsmaßnahmen inklusive Informationskampagnen, wie sie im fünften Kapitel angeregt wurden. Darüber hinaus muß bedacht werden, daß der Wirkungsgrad von Instrumenten auch von der Abstimmung mit anderen, dazu komplementären Maßnahmen abhängt (vgl. Orszag/Snower 1998). Auf die hier vorgeschlagenen Lohnsubventionen bezogen heißt dies, daß die auf den Güter- und Dienstleistungsmärkten vorhandenen Regulierungen dahingehend $z u$ prüfen sind, ob sie die Schaffung eines transfergestützten Niedriglohnsegmentes unterstützen oder eher behindern.

Die Zielgruppenbezogenheit der Lohnsubventionen erhöht zwar ihre Effektivität, generiert aber eine horizontale Ungerechtigkeit, da nur diejenigen in den Genuß der Subvention kommen, die den Status der Zielgruppe besitzen. Alle anderen jedoch, die aus der stillen Reserve heraus einen Job in diesem Niedriglohnbereich suchen, werden dadurch systematisch benachteiligt. Dies würde dafür sprechen, daß die Subventionsleistung sich langfristig nicht am Arbeitnehmer, sondern am Erwerbseinkommen orientiert.

Akzeptiert man den letzten Punkt, so sollte man im Zuge einer sowieso anstehenden und dringend notwendigen Steuerreform die monetäre Unterstützung von Niedriglohnbeziehern nicht mehr über Subventionen, sondern über das 
Steuersystem durchführen, wie dies im Falle einer negativen Einkommensteuer möglich wäre. Denn insbesondere die in der Sozialhilfe zum Ausdruck kommenden familienpolitischen Leistungen und Leitbilder sowie die damit verbundenen negativen Arbeitsanreize können durch eine Lohnsubvention nicht oder nur ungenügend aufgefangen werden - wohl aber mit einer Steuerlösung. Ebenso könnten in einem derartigen Rahmen die Probleme unterschiedlicher Be- und Entlastungen der verschiedenen Institutionen besser koordiniert werden. Dafür wäre allerdings eine bundesweite Reform notwendig. 


\section{Literatur}

Adil, M. (1998): Qualifizierende Arbeitsvermittlung, in: Blätter der Wohlfahrtspflege - Deutsche Zeitschrift für Sozialarbeit 7+8/98, Stuttgart, 159-160.

Albeck, H. (1982): Lohnsubventionen als Mittel der Arbeitsmarktpolitik, in: Herder-Dorneich, Ph. (Hrsg.): Arbeitsmarkt und Arbeitsmarktpolitik, Berlin.

Andel, N. (1970): Subventionen als Instrument des finanzwirtschaftlichen Interventionismus, Tübingen.

Andreß, H.-J. / Strengmann, W. (1997): Warum arbeiten, wenn der Staat zahlt? Über das Arbeitsangebot unterer Einkommensschichten, in: Zeitschrift für Sozialreform 43, 505-525.

BMWi (1998): Beschäftigungspolitischer Aktionsplan der Bundesrepublik Deutschland, Bonn.

Böckmann-Schewe, L. / Röhrig, A. (1997): "Hilfe zur Arbeit" - Analyse der Wirksamkeit öffentlich geförderter Beschäftigung für Sozialhilfeempfängerlnnen - Abschlußbericht des Forschungsprojektes Berlin-Brandenburg Institut für Sozialforschung und sozialwissenschaftliche Praxis e.V. (BIS), Graue Reihe - Neue Folge 131, Hans-BöcklerStiftung, Düsseldorf.

Bohlen, C. (1993): Zur Theorie und Empirie von Lohnsubventionen. Ein Beitrag zur Analyse der ökonomischen Wirkungen sozialrechtlicher Normen und zur Arbeitsmarktpolitik in der Bundesrepublik Deutschland 1980 bis 1989, Berlin.

Bosch, G. u.a. (1994): Der zweite Arbeitsmarkt: Perspektiven und Chancen, in: Daheim, H. u.a. (Hrsg.): Standortfaktor industrielle Beziehungen, München u.a.

Brennecke, J. (1996): Zur Ermittlung des Erwerbspersonenpotentials in der Sozialhilfe, in: Landeshauptstadt Wiesbaden Sozialdezernat (Hrsg.): Armut und Sozialhilfe im Rhein-Main-Gebiet, Wiesbaden, 44-56.

Bundesinstitut für Berufsbildung (1995): Berufsbegleitende Nachqualifizierung, Ergebnisse, Veröffentlichungen und Materialien aus dem BiBB, Berlin/Bonn.

Cornetz, W. I Schäfer, H. (1998): Hat Deutschland den Dienstleistungsrückstand gegenüber den USA aufgeholt? in: Wirtschaftsdienst VII/98, 418-425.

Ehrlich, V. (1997): Arbeitslosigkeit und Zweiter Arbeitsmarkt. Theoretische Grundlagen, Probleme und Erfahrungen, Frankfurt am Main u.a. 
Emmerich, K. (1998): Dänemark: Arbeitsmarktflexibilität bei hoher sozialer Sicherung, in: Wirtschaftsdienst 1998/VII, 401-406.

Emmerich, K. (1998): Dänemark: Arbeitsmarktflexibilität bei hoher sozialer sicherung, in: Wirtschaftsdienst 1998/VII, 401-406.

Freeman, R. / Gottschalk, P. (eds.) (1998): Generating Jobs. How to increase demand for less-skilled workers, New York.

G.I.B. - Gesellschaft für innovative Beschäftigungsförderung gGmbH (1998): Arbeitsmarktintegration von Sozialhilfebeziehenden - Aktivitäten der Kommunen in Nordrhein-Westfalen, G.I.B. Arbeitspapiere 3, Bottrop.

Gemeinnützige Offenbacher Ausbildungs- und Beschäftigungs mbH (1998): Jahresbericht der Geschäftsführung für das Jahr 1997, in: GOAB-Dokumentation, Berichte - Konzepte - Arbeitspapiere, Heft 9, Öfentliche Konzepte und Berichte 1997, Offenbach.

Hackenberg, H. / Wagner, G. (1996): Kommunale Beschäftigungsförderung im Spannungsfeld von Innovation, Mißbrauchsrhetorik und Reform der Sozialhilfe, in: WSI-Mitteilungen $12 / 96,774-782$.

Hartmann, H. (1998): Arbeitslosenhilfe und Sozialhilfe - Zwei Systeme für das gleiche soziale Problem? in: Nachrichtendienst des Deutschen Vereins 1/98, 9-12.

Hessisches Statistisches Landesamt (1998): Die Sozialhilfe in Hessen im Jahr 1996, Teil 2: Empfänger, Wiesbaden.

Hessisches Statistisches Landesamt (i.E.): Die Sozialhilfe in Hessen im Jahr 1997, Teil 2: Empfänger, Wiesbaden.

Kehler, I. (1997): Ein neuer Beitrag zur kommunalen Arbeitsmarktpolitik, in: Der Städtetag, Heft 5, Köln, 345-346.

Kehler, I. (1998): 1.000 Ressourcen - Evaluation eines innovativen Arbeitsvermittlungsprojektes, INITA Institut für Angewandte Psychologie, Transaktionsanalyse und Erwachsenenbildung, Hannover.

Klopfleisch, R. / Sesselmeier, W. / Setzer, M. (1997a): Beschäftigungspolitische Wirkungen einer Negativen Einkommensteuer, in: Konjunkturpolitik 3/97, 223-247.

Klopfleisch, R. / Sesselmeier, W. / Setzer, M. (1997b): Wirksame Instrumente einer Arbeitsmarkt- und Beschäftigungspolitik, in: Aus Politik und Zeitgeschichte B 35/97, 23-32.

Klös, H.-P. (1997a): Dienstleistungslücke und Niedriglohnsektor in Deutschland, in: iwtrends $3 / 97,33-59$. 
Klös, H.-P. (1997b): Einfacharbeitsplätze. Stiefkinder des Arbeitsmarktes, Institut der deutschen Wirtschaft, Köln.

Klös, H.-P. (1998): Arbeit plus Transfers. Zur Reform der kommunalen Beschäftigungspolitik, Beiträge zur Wirtschafts- und Sozialpolitik Band 243, Köln.

Kress, U. (1994): Der zweite Arbeitsmarkt in der Diskussion, IAB-Werkstattbericht Nr. 5, Nürnberg.

Leibfried, S. / Leisering, L. u.a. (1995): Zeit der Armut, Frankfurt/M.

Löwisch, M. (1998): Durchaus einen Versuch wert. Kombi-Lohn im Arbeitslosengeld/-hilfe, in: Arbeit und Arbeitsrecht 4/98, 119-120.

Miethe, H. / Brödnow, J. (1993): Meinungen, Erfahrungen und Erwartungen von ABM-Beschäftigten, IAB-Werkstattbericht Nr. 20, Nürnberg.

OECD (1993): Employment Outlook, Paris.

Orszag, M. / Snower, D. (1998): Anatomy of Policy Complementarities, CEPR Discussion Paper No. 1963, London.

Phelps, E. (1997): Rewarding Work. How to Restore Participation and Self-Suport to Free Enterprise, Cambridge/Mass.

Presse- und Informationsamt der Bundesregierung (1997): Wohngeld, Bonn.

Scharpf, F. (1994): Nicht Arbeitslosigkeit, sondern Beschäftigung fördern, in: Meyer, H.-W. (Hrsg.): Sozial gerecht teilen - ökologisch umsteuern? Beiträge zur Reformdiskussion im Deutschen Gewerkschaftsbund und seinen Gewerkschaften, Band 2, Köln, 24-42.

Schellhaaß, H.-M. / Kelp, L. (1995): Subventionen als Steuerungsinstrumente des Arbeitsmarktes, in: MittAB 28, 399-412.

Schettkat, R. (1998): Arbeitsmarktinstitutionen und Beschäftigung bei unterschiedlichen Wachstumsregimen, in: Lessenich, S. I Ostner, I. (Hrsg.): Welten des Wohlfahrtskapitalismus. Der Sozialstaat in vergleichender Perspektive, Frankfurt/New York, 201-224.

Schomburg, J. (1994): Arbeitsvermittlung aus arbeitsfördernden Maßnahmen, in: SchulzeBöing, M. (Hrsg.): Die Zukunft des Arbeitsmarktes - Institutionenreform und neue Wege in der Arbeitsmarktpolitik, Graue Reihe - Neue Folge 72, Hans-Böckler-Stiftung, Düsseldorf, 156-160.

Schulze-Böing, M. / Simon, H. (1998): Arbeitsvermittlung als soziale Wertschöpfung - Elemente eines integrierten Beratungs- und Vermittlungskonzepts, Materialien zur Arbeitsförderung 1, Stadt Offenbach am Main: Amt für Arbeitsförderung und Statistik. 
Sesselmeier, W. (1997): Einkommenstransfers als Instrumente der Beschäftigungspolitik. Negative Einkommensteuer und Lohnsubventionen im Lichte der modernen Arbeitsmarkttheorien und der Neuen Institutionenökonomik, Frankfurt am Main u.a.

Sesselmeier, W. (1998): Negative Einkommensteuer und Gerechtigkeit, in: Blasche, S. I Döring, D. (Hrsg.): Sozialpolitik und Gerechtigkeit, Frankfurt/New York, 356-383.

Sesselmeier, W. I Klopfleisch, R. I Setzer, M. (1996): Mehr Beschäftigung durch eine negative Einkommensteuer. Zur beschäftigungspolitischen Effektivität und Effizienz eines integrierten Steuer-Transfer-Systems, Frankfurt am Main u.a.

Setzer, M. I Cullmann, O. (1996): Globalization and Welfare. Consequences for Employment, Income Distribution and Social Security, Forschungsprojekt: Ökonomische Dimensionen, Bereiche und Indikatoren der Globalisierung, Arbeitspapier Nr. 4, TU Darmstadt.

SiT-Selbsthilfe im Taunus e.V. (1997): Auszug aus dem Jahresbericht, Hofheim/Ts.

Sperling, I. (1994): Probleme des zweiten Arbeitsmarktes, in: Wirtschaftsdienst 74, 398-402.

Spitznagel, E. (1992): Allgemeine Maßnahmen zur Arbeitsbeschaffung in den neuen Bundesländern, in: Mitteilungen aus der Arbeitsmarkt und Berufsforschung 25, 277.

Stadt Darmstadt (1998): Beschäftigungsförderung - Jahresbericht 1997, Dezernat Sozialverwaltung/Beschäftigungsförderung.

Stadt Offenbach am Main (1998a): FAMS - Fallmanagement in der Sozialhilfe, Projektbericht.

Stadt Offenbach am Main (1998b): Kommunale Arbeitsförderung - Hilfe zur Arbeit - Arbeitsbericht 1996 und 1997, Materialien zur Arbeitsförderung 2, Stadt Offenbach am Main: Amt für Arbeitsförderung und Statistik.

Trabert, L. et al. (1998): Kombilohn in Sachsen-Anhalt, in: Ministerium für Arbeit, Frauen, Gesundheit und Soziales des Landes Sachsen-Anhalt (Hrsg.): Kombilohn in Sachsen-Anhalt. Gutachten zu den erwarteten fiskalischen Wirkungen des Vorschlags des Bundesministeriums für Gesundheit zu den Freibeträgen bei der Anrechnung von Erwerbseinkommen auf die Sozialhilfe, Forschungsbeiträge zum Arbeitsmarkt in Sachsen-Anhalt Band 12, Magdeburg, 9-42.

Vanselow, A. / Weinkopf, C. (1996): Sozialverträgliche Arbeitnehmerüberlassung als arbeitsmarktpolitisches Instrument - die bisherigen Erfahrungen mit START Zeitarbeit NRW, Erster Zwischenbericht der fachlichen Begleitung von Juli 1996, Projektbericht des Instituts Arbeit und Technik 1996/11, Gelsenkirchen.

Vanselow, A. / Weinkopf, C. (1998): START Zeitarbeit NRW zwischen Sozialverträglichkeit und Wirtschaftlichkeit - Zweiter Zwischenbericht der fachlichen Begleitung im Auf- 
trag des Ministeriums für Arbeit, Gesundheit und Soziales des Landes NordrheinWestfalen, Projektbericht des Instituts Arbeit und Technik 1998-03, Gelsenkirchen.

Vierling, M. (1997): Subventionierung des Lohnsatzes anstelle des Einkommens, in: Wirtschaftsdienst XI/97, 647-654.

Vierling, M. (1998): Kombilohn: Die Frage ist nicht "ob", sondern „wie", in: Wirtschaftsdienst 1998/IX, 529-535.

Wagner, G. / Stangl, J. (1998): Kombilohn in Sachsen-Anhalt, in: Ministerium für Arbeit, Frauen, Gesundheit und Soziales des Landes Sachsen-Anhalt (Hrsg.): Kombilohn in Sachsen-Anhalt. Gutachten zu den erwarteten fiskalischen Wirkungen des Vorschlags des Bundesministeriums für Gesundheit zu den Freibeträgen bei der Anrechnung von Erwerbseinkommen auf die Sozialhilfe, Forschungsbeiträge zum Arbeitsmarkt in Sachsen-Anhalt Band 12, Magdeburg, 43-107.

Werner, H. (1998): Beschäftigungspolitisch erfolgreiche Länder - Was steckt dahinter?, in: Mitteilungen aus der Arbeitsmarkt- und Berufsforschung 31, 324-333.

Wilk, Ch. (1997a): Wege aus der Sozialhilfe, in: Sozialer Fortschritt 12/97, 284-289.

Wilk, Ch. (1997b): Beschäftigungsprojekte zwischen Subvention und Innovation, Frankfurt u.a.

Wirtschaftsförderung Region Frankfurt / Rhein-Main e.V. (1998): Projekte zur Arbeitsmarktund Beschäftigungsförderung in der Region RheinMain, Umlandverband Frankfurt.

WSI (1998): WSI-Tarifhandbuch 1998, Köln.

Zukunftskommission der Friedrich-Ebert-Stiftung (1998): Wirtschaftliche Leistungsfähigkeit, sozialer Zusammenhalt, ökologische Nachhaltigkeit. Drei Ziele - ein Weg, Bonn. 
Martin Setzer, Roland Klopfleisch and Werner Sesselmeier - 978-3-631-75277-7

Downloaded from PubFactory at 01/11/2019 06:22:32AM

via free access 


\section{Anhang}

Martin Setzer, Roland Klopfleisch and Werner Sesselmeier - 978-3-631-75277-7

Downloaded from PubFactory at 01/11/2019 06:22:32AM

via free access 


\section{Unterscheidungs- und Erfolgskriterien von Beschäftigungsinitiativen}

Ein wertender Vergleich von Beschäftigungsinitiativen ist praktisch schwer durchführbar. Vermittlungserfolge hängen zum Großteil vom regionalen wirtschaftlichen und politischen Umfeld ab, so daß eine Aussage, diese oder jene Methode sei erfolgreicher als die anderen, nur bedingt zutreffend ist. Darüber hinaus existieren eine Vielzahl von kaum meßbaren Faktoren, welche jedoch mit Sicherheit als Folge von erfolgreicher Vermittlungsarbeit zu sehen sind (bspw. Rückgang von Kriminalität, Reduzierung der medizinischen und psychologischen Behandlungskosten, Verbesserung des sozialen Umfeldes, etc.). Die nachfolgende tabellarische Auflistung verschiedener Initiativen besitzt dementsprechend einen eher informativen denn vergleichenden Charakter. Gleichzeitig sind dennoch in dieser Übersicht unabhängig vom regionalen Umfeld, Basisbausteine erfolgreicher Vermittlung bei allen aufgeführten Initiativen zu erkennen. Als besonders effektiv erweisen sich Methoden, welche sowohl Beschäftigungs-, als auch Qualifizierungs- und Sozialbetreuungsmaßnahmen beinhalten.

Zunächst folgt eine mit erläuternden Beispielen versehene Aufstellung aller in den Tabellen verwandten Untersuchungskritieren:

\section{Projektstrukturdaten:}

- Trägerschaft

(Wohlfahrtspflege, Kommune, freie Trägerschaft)

- Rechtsform

(gemeinnützige $\mathrm{GmbH}$, e.V. mit enger oder loser Anbindung an einen Verband, gemeinnützige Stiftung)

- Finanzierungsquellen

(Mittel der öffentlichen Haushalte zuzüglich Fördermittel (EU, Bund, Land), Eigenmittel der Beschäftigungsträger, erwirtschaftete Erlöse, Spenden)

- Personal

(Stammpersonal der Beschäftigungsinitiativen: Anzahl, Vollzeit/Teilzeit, befristet/unbefristet)

- Größe

(Anzahl der Maßnahmeteilnehmer) 
- Aufgabenfelder

(In welche Bereiche des Ersten und Zweiten Arbeitsmarktes vermittelt die Beschäftigungsinitiative? Sind Eigenbetriebe vorhanden?)

- Angebote:

- Dauer der Beschäftigung

(Verbleibdauer der Teilnehmer in Maßnahmen der Beschäftigungsinitiative)

- Entlohnung

(tariflich, ortsüblich; ergänzender oder voller Bezug von Sozialhilfe)

- TeilzeitNollzeit

- Teilnehmergewinnung

(über das Sozialamt, über das Arbeitsamt, durch Ausschreibungen, durch Eigeninitiative, durch persönliche Kontakte)

- Beratung

(Persönlichkeitsstabilisierung, psychische Betreuung, Schuldnerberatung, Familienberatung, Motivationstraining, Kommunikationstraining. Hilfe bei Behördengängen, Unterstützung bei der Stellensuche durch Bewerbungstraining, ggí. Beraiung bei Fragen der Freizeitgestaitung)

- Orientierung

(Übergang von Beratung zu Orientierung oft fließend; spezielle Orientierungsmaßnahmen sind bspw. Hilfeplanung, Maßnahmenplanung, Berufswegeplanung)

- Qualifizierung

(Schlüsselqualifizierungen, weitergehende Qualifizierungen mit und ohne Abschlüsse)

- Beschäftigung (auf dem Zweiten Arbeitsmarkt in Eigenbetrieben oder bei kooperierenden Stellen nach verschiedenen Varianten der $\S \S 18-20$ BSHG)

- Vermittlung (Konzept der Vermittlung)

- Betreuung

(intern oder extern; vor, während und nach der Vermittlung in Maßnahmen, Beschäftigung oder reguläre Arbeit) 
- Instrumente

(vermittlungsunterstützende Instrumente, bspw. Praktika, Zeitarbeit, Arbeitnehmerüberlassung)

\section{Erfolgskriterien:}

- Erreichen der Zielgruppe

(Werden Langzeitarbeitslose wirklich eingebunden, welche Gruppen bleiben außen vor?)

- Integrationsleistungen

- Projektintegration (Übergang aus der Arbeitslosigkeit in das Projekt, in die Maßnahme)

- Qualifizierungsphase (Voraussetzungen zur Vermittlung schaffen)

- Arbeitsmarktintegration (Übergang aus den Maßnahmen der Beschäftigungsinitiative in ein Arbeitsverhältnis)

- Beschäftigungswirkungen

(Situation der Maßnahmenabsolventen sechs bzw. zwölf Monate nach dem Maßnahmenende hinsichtlich Sozialhilfebezug (voll, ergänzend, ohne) und Arbeitssituation (Erster oder Zweiter Arbeitsmarkt))

\section{- Kosten}

Verhältnis fiskalische Entlastungswirkung zu quantifizierbaren Brutto- und Nettokosten

Anhand obiger Kriterien werden nun die folgenden sechs Beschäftigungsinitiativen untersucht:

1. GOAB

2. Fallmanagement Sozialamt Offenbach

3. SiT - Selbsthilfe im Taunus

4. START Zeitarbeit NRW

5. Projekt 1.000 Ressourcen

6. Internationaler Bund Darmstadt 


\begin{tabular}{|c|c|}
\hline Kriterium & GOAB \\
\hline \multicolumn{2}{|c|}{ Projektstrukturdaten } \\
\hline Trägerschaft & Kommunale Beschäftigungsgesellschaft der Stadt Offenbach \\
\hline Rechtsform & Gemeinnützige $\mathrm{GmbH}$ \\
\hline Finanzierungsquellen & $\begin{array}{l}\text { - Zuschüsse der Stadt Offenbach } \\
\text { - Zuschüsse der Arbeitsverwaltung } \\
\text { - Zuschüsse aus Mitteln des Europäischen Sozialfonds (ESF) } \\
\text { - Zuschüsse des Landes Hessen } \\
\text { - Zuschüsse des Kreises Offenbach } \\
\text { - Zuschüsse des Landeswohlfahrtsverbandes } \\
\text { - Erlöse aus dem Eigenbetrieb } \\
\text { - Evtl. Investitionszuschüsse }\end{array}$ \\
\hline Personal & Gesamtgesellschaft derzeit durchschnittlich 300 Personen $^{2}$ \\
\hline Größe & $\begin{array}{l}\text { - ca. } 170 \text { Teilnehmer in Maßnahmen und Arbeitnehmerüberlassung }{ }^{3} \\
\text { - In einem gestuften Verfahren werden nahezu alle arbeitsfähigen Sozialhilfeempfänger } \\
\text { erfaßt und zur Arbeitsberatung eingeladen }{ }^{4} \text {. }\end{array}$ \\
\hline Aufgabenfelder & $\begin{array}{l}\text { Die GOAB beschäftigt oder vermittelt befristete Arbeit in folgenden Bereichen. Die Be- } \\
\text { schäftigung findet im Rahmen von ABM- und HzA-Maßnahmen statt. } \\
\text { - Landschaftsbau } \\
\text { - Renovierung (Schulsanierung) } \\
\text { - Bau } \\
\text { - Recyclingzentrum } \\
\text { - Pflege und Betreuung (ambulante Hilfen, Familienhilfe, Kinderbetreuung) }\end{array}$ \\
\hline
\end{tabular}

\footnotetext{
${ }_{1}^{1}$ alle Finanzierungsquellen vgl:: Gemeinnützige Offenbacher Ausbildungs- und Beschäftigungs mbH $(1998,4)$

${ }^{2}$ Vgl.: Gemeinnützige Offenbacher Ausbildungs- und Beschäftigungs mbH $(1998,1)$ sowie Böckmann-Schewe, Lisa/Röhrig, Anne $(1997,125)$

${ }^{3}$ Vgl:: Gemeinnützige Offenbacher Ausbildungs- und Beschäftigungs mbH $(1998,6)$

${ }^{4}$ Vgl.: Stadt Offenbach am Main (1998b, 3)
} 


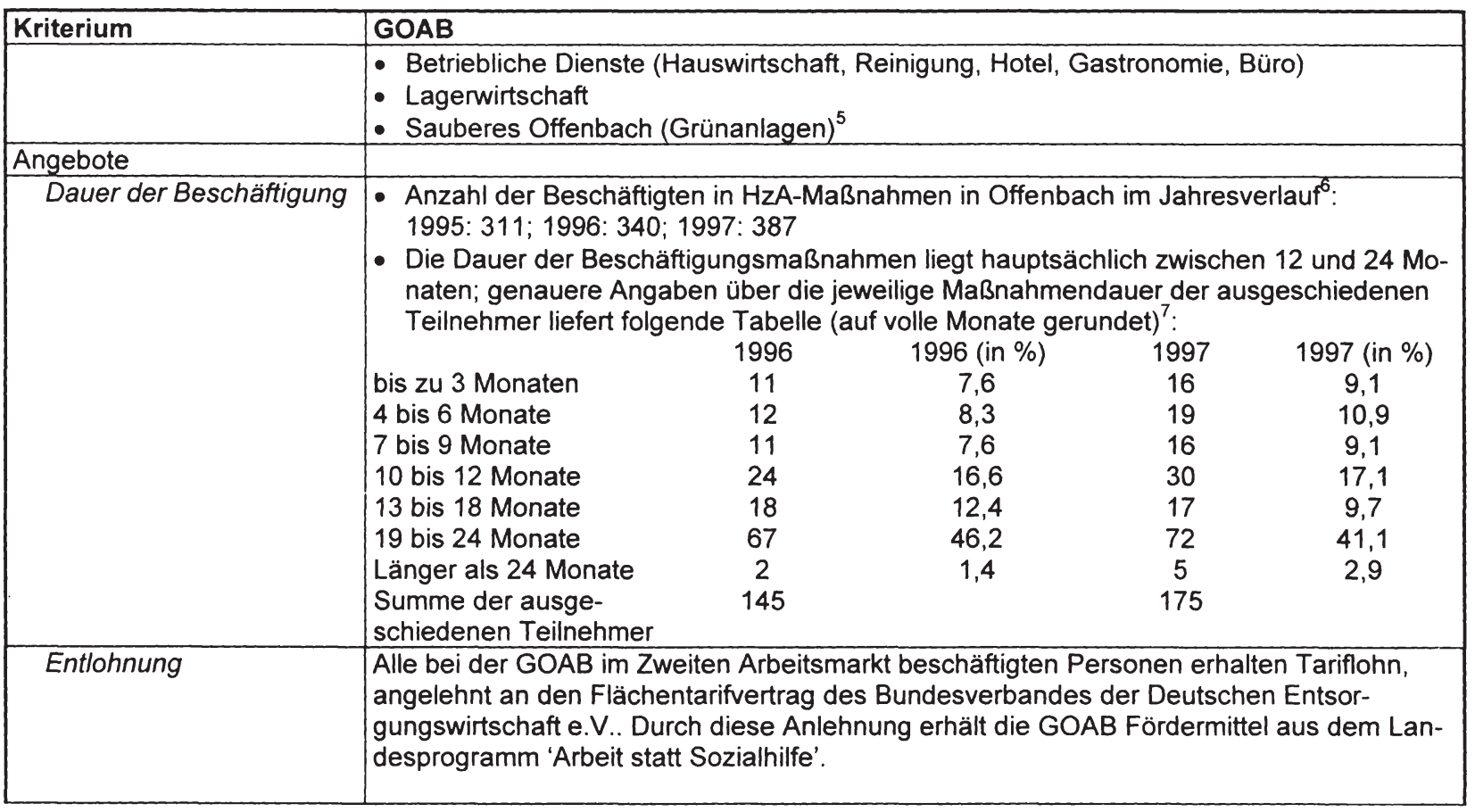

5 alle Aufgaben vgl.: Gemeinnützige Offenbacher Ausbildungs- und Beschäftigungs mbH $(1998,19)$

${ }^{6}$ Daten zu 1995 vgl. Böckmann-Schewe, Lisa/Röhrig. Anne (1997, 125); zu 1996 und 1997 vgl. Stadt Offenbach am Main $(1998 b, 14)$

${ }^{7} \mathrm{Vgl} .:$ Stadt Offenbach am Main $(1998 b, 15)$ 


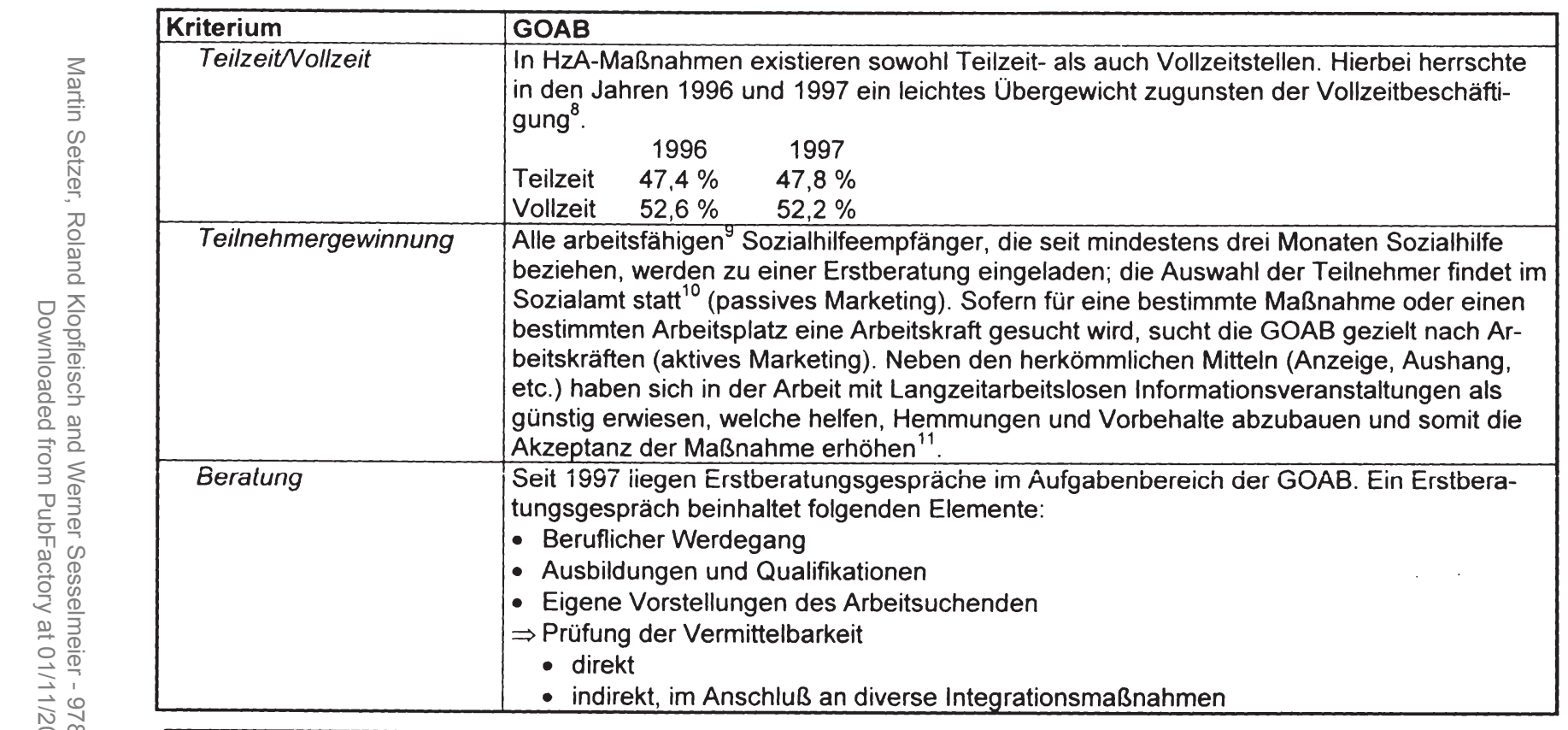

\footnotetext{
${ }_{9}^{8} \mathrm{Vgl.:}$ Stadt Offenbach am Main (1998b, 15) 6.1

${ }_{11}^{11}$ Vgl.: Böckmann-Schewe, Lisa/Röhrig, Anne $(1997,124)$

${ }^{11}$ Vgl.: Schulze-Böing, M/Simon, H. $(1998,13 f$.
}

${ }^{9}$ Als nicht arbeitsfähig werden Personen mit gesundheitlichen Beeinträchtigungen - körperlich und psychisch -, mit Suchtproblemen und Frauen in Schwangerschaft oder mit betreuungspflichtigen Kindern eingestuft. Zur Abgrenzung s. 


\begin{tabular}{|c|c|}
\hline Kriterium & GOAB \\
\hline & $\begin{array}{l}\text { - Sozialversicherungspflichtige Maßnahmen } \\
\text { - Orientierungskurse } \\
\text { - Praktika } \\
\Rightarrow \text { Sofern keine direkte Vermittlung möglich ist, schließen sich in der Regel zwei bis vier } \\
\text { weitere Beratungsgespräche an das Erstberatungsgespräch an, um Art und Reihenfol- } \\
\text { ge der Integrationsmaßnahmen festzulegen (siehe auch: Orientierung). } \\
\text { Seit } 1989 \text { existiert bei der GOAB eine Schuldnerberatungsstelle, die seit dem 01.07.1998 } \\
\text { ausschließlich für Angestellte der GOAB zuständig ist. Das Beratungsangebot der Stadt } \\
\text { führt seitdem das Diakonische Werk weiter. Sofern die bei der GOAB zumeist nur befristet } \\
\text { angestellten Schuldner über ihr Beschäftigungsverhältnis hinaus verschuldet bleiben, } \\
\text { müssen sie seit diesem Sommer einen Beraterwechsel in Kauf nehmen. }\end{array}$ \\
\hline Orientierung & $\begin{array}{l}\text { - Orientierungslehrgänge existieren seit } 1992 \\
\text { - Dauer zwei Monate, als Vorstufe zu einer HzA-Maßnahme } \\
\text { - Ziele der Orientierungslehrgänge sind: } \\
\text { - Falldiagnose erstellen } \\
\text { - Lebenspläne klären und evaluieren } \\
\text { - Hilfeplan entwickeln } \\
\text { - Orientierungswissen vermitteln } \\
\text { - Praxiserprobung durchführen } \\
\text { - Praktische Grundqualifikationen vermitteln } \\
\text { - Entscheidung über den weiteren Weg heraus aus der Sozialhilfe treffen } \\
\Rightarrow \text { Umsetzung dieser Ziele durch: } \\
\text { - Einzelberatung (die schon oben unter Beratung erwähnten vertiefenden zwei bis vier } \\
\text { - Beratungsgespräche) } \\
\text { - Gruppenunterricht } \\
\text { - Betriebspraktika }\end{array}$ \\
\hline
\end{tabular}




\begin{tabular}{|l|l|}
\hline Kriterium & GOAB \\
\hline & $\begin{array}{l}\text { Techniken der aktiven Arbeitsvermittlung } \\
\text { Ablauf }\end{array}$ \\
1. drei Wochen Orientierungslehrgang mit den angesprochenen vertiefenden Einzelge- \\
sprächen \\
2. mindestens drei Wochen Arbeitserprobung (Praktikum) \\
3. eine Woche Auswertung mit individueller Hilfeplanung bzw. Arbeitsvermittlung \\
Über das Amt für Arbeitsförderung und Statistik (siehe: Sonstiges, Punkt 2) gelangen die \\
Teilnehmer in die Orientierungskurse, die GOAB führt die Lehrgangsteile durch, die Prak- \\
tika absolvieren die Teilnehmer entweder bei der GOAB, bei anderen Trägern oder bei \\
einem Privatunternehmen
\end{tabular}

${ }^{12}$ Vgl.: Schulze-Böing, M/Simon, H. $(1998,22 f$.)

${ }^{13} \mathrm{Vgl}$ :: Böckmann-Schewe, Lisa/Röhrig, Anne (1997, 124f.)

${ }^{14}$ Eigenbetrieb der Stadt Offenbach (ESO)

${ }^{15}$ alle Qualifizierungsarten vgl.: Gemeinnützige Offenbacher Ausbildungs- und Beschäftigungs mbH $(1998,8)$ 


\begin{tabular}{|c|l|}
\hline Kriterium & GOAB \\
\hline Vermittlung & Vermittlung im Sinne der GOAB beinhaltet die folgenden drei Komponenten: \\
1. Funktionierender Stellenpool & Zu \\
Zu einem funktionierenden Stellenpool gehören sowohl eine Stellenakquise zum Auf- \\
spüren von freien Stellen und Erstellen von entsprechenden Anforderungsprofilen als \\
auch eine Marketingstrategie, um die Reputation der Beschäftigungsgesellschaft bei \\
den jeweiligen Unternehmen zu erhöhen. Der Arbeitsauftrag, die Projektzusammen- \\
hänge und die zu vermittelnde Zielgruppe schränken die Suche nach offenen Stellen \\
ein. Folgende Schritte sind beim Aufbau eines Stellenpools durchzuführen: \\
- Marktbeobachtung \\
- Telefonkontakt \\
- Betriebsbesuch \\
- Dokumentation (Informationsverarbeitung) \\
2. Personalauswahl (siehe auch: Teilnehmergewinnung) \\
Die GOAB verfolgt bei der Personalauswahl einen ganzheitlichen Ansatz, indem sie \\
sowohl die fachlichen und arbeitsplatzbezogenen Anforderungen und Fähigkeiten als \\
auch die Lebenslage des Arbeitsuchenden berücksichtigt. Von entscheidender Bedeu- \\
tung in diesem Zusammenhang ist die Frage, wie der Arbeitslose mit seiner Situation \\
umgeht. Nach den vorgeschalteten Stufen der Teilnehmergewinnung, bedarf es einer \\
schriftlichen Bewerbung des Arbeitsuchenden. Anschließend erfolgen Beratungsge- \\
spräche, welche gegebenenfalls von Orientierungs-, Beschäftigungs- und Qualifizie- \\
rungsmaßnahmen gefolgt werden (siehe auch: Beratung, Orientierung, Beschäftigung, \\
Qualifizierungen). \\
3. systematisches Vorgehen bei der Vermittlung selbst ${ }^{17}$ \\
Die GOAB versucht bei der Vermittlung sowohl die objektive Komponente \\
(Qualifikations- und Verhaltensanforderungen) als auch die subjektive Dimension
\end{tabular}

${ }^{16} \mathrm{Vgl}$.: Schulze-Böing, M./Simon, H. (1998, 6ff.)
${ }^{17}$ Vgl.: Schulze-Böing, M./Simon, H. (1998, 24ff.) 


\begin{tabular}{|c|c|c|}
\hline \multirow{2}{*}{\multicolumn{2}{|c|}{$\begin{array}{ll}3 & \text { Kriterium } \\
& \end{array}$}} & GOAB \\
\hline & & $\begin{array}{l}\text { (Eigenschafts- und Fähigkeitsanforderungen) des Arbeitsuchenden zu berücksichti- } \\
\text { gen }{ }^{18} \text {. Sie stellt dem Unternehmen alle ihrer Meinung nach passenden Bewerber mit in- } \\
\text { ren Stärken und Schwächen kurz vor und gibt ggf. eine Empfehlung. Die letztendliche } \\
\text { Wahl verbleibt beim Unternehmen. Eine Vermittlung kann durch folgende Möglichkeiten } \\
\text { unterstützt werden: } \\
\text { - assistiertes Bewerbungsgespräch } \\
\text { - Erprobung der Arbeitskraft durch ein Praktikum } \\
\text { - Beratung über Lohnkostenzuschüsse, ggf. Angebot, selbst Lohnkostenzuschüsse zu } \\
\text { finanzieren }\end{array}$ \\
\hline & Betreuung & $\begin{array}{l}\text { Eine Nachbetreuung wird als fester Bestandteil bei der Arbeitsvermittlung eingeplant, an- } \\
\text { geboten und bei Bedarf realisiert. }\end{array}$ \\
\hline & Sonstiges & $\begin{array}{l}\text { 1. Die GOAB führt im Rahmen ihres Projekts "matchwork" (ehemals EU-gefördert) bun- } \\
\text { desweit Schulungen für Personaldisponenten durch. Das Schulungskonzept basiert auf } \\
\text { der Maatwerk-Methode. } \\
\text { 2. Die GOAB arbeitet eng mit dem Amt für Arbeitsförderung und Statistik zusammen, wel- } \\
\text { ches } 1995 \text { aus dem Sachgebiet "Hilfe zur Arbeit" im Offenbacher Sozialamt hervorging. } \\
\text { Das Amt für Arbeitsförderung und Statistik ist direkt dem Oberbürgermeister unterstellt. } \\
\text { Die Vorteile der Ausgliederung liegen einerseits in einem Anerkennungszuwachs ge- } \\
\text { genüber anderen Behörden und andererseits in einer erhöhten Kooperationsbereit- } \\
\text { schaft der Hilfeempfänger aufgrund der Trennung von Leistungsbezug und Beschäfti- } \\
\text { gungsträger. } \\
\text { Das Amt verfolgt folgende Ziele: } \\
\text { - Beratung der Maßnahmeträger (alle Informationen über Träger und Maßnahmen } \\
\text { fließen hier zusammen) } \\
\text { - Unterstützung und Hilfe für Sozialhilfeempfänger }\end{array}$ \\
\hline
\end{tabular}

${ }^{18}$ Vgl.: Schulze-Böing, M./Simon, H. $(1998,24)$ 


\begin{tabular}{|c|c|}
\hline Kriterium & GOAB \\
\hline & $\begin{array}{l}\text { - Steuerung der "Hilfe zur Arbeit" } \\
\text { 3. Entwicklung neuer Maßnahmen" } \\
\text { uie GOAB kooperiert seit } 1996 \text { mit der 'Arbeitsgemeinschaft Unternehmensgründung', } \\
\text { um Arbeitsuchende beim Aufbau einer eigenen Existenz zu unterstützen. Die Unter- } \\
\text { stützung beinhaltet folgende Tätigkeiten: } \\
\text { - Geeignete Personen auswählen und beraten } \\
\text { - Geschäftspläne mitentwickeln } \\
\text { - evtl. neue Kleinunternehmen in der Gründungsphase beraten }{ }^{20} \\
\text { Seit Beginn der Kooperation wurde ein Unternehmen gegründet, welches seit andert- } \\
\text { halb Jahren besteht (Stand Mai 1998); Zuschüsse oder Bürgschaften der Stadt nahm } \\
\text { das gegründete Unternehmen nicht in Anspruch. Eine weitere Existenzgründung steht } \\
\text { in nächster Zeit bevor. } \\
\text { "Das Potential wirklich erfolgversprechender Fälle wird auf 0,5 Prozent aller arbeitsfähi- } \\
\text { gen Hilfeempfänger veranschlagt. Aber auch dies wären in Offenbach immer noch etwa } \\
\text { 10-15 Fälle. Wenn man diesen Menschen mit vertretbarem Aufwand helfen kann, ist } \\
\text { das eine durchaus sinnvolle Ergänzung von Beschäftigungsmaßnahmen"21. }\end{array}$ \\
\hline \multicolumn{2}{|l|}{ Erfolgskriterien } \\
\hline Erreichen der Zielgruppe & $\begin{array}{l}1.200 \text { bis } 1.400 \text { Sozialhilfeempfänger werden pro Jahr zum Erstberatungsgespräch einge- } \\
\text { laden ( } 30 \text { bis } 50 \% \text { von insgesamt ca. } 3.000 \text { Sozialhilfeempfängern in Offenbach) }\end{array}$ \\
\hline \multicolumn{2}{|l|}{ Integrationsleistungen } \\
\hline Projektintegration & $\begin{array}{l}1997 \text { lud die GOAB ca. } 1.500 \text { Sozialhilfeempfänger zu Beratungsgesprächen ein, davon } \\
\text { fanden } 900 \text { tatsächlich statt. Zusätzlich waren alle Neuantragsteller ab Mitte des Jahres } \\
\text { verpflichtet, an einem Erstberatungsgespräch teilzunehmen. Dies ergibt eine Quote von }\end{array}$ \\
\hline
\end{tabular}

${ }^{19}$ Vgl.: Böckmann-Schewe, Lisa/Röhrig, Anne $(1997,123)$

${ }^{20}$ Die Beratung wird durch das Hessische Wirtschaftsministerium bezuschußt.

${ }^{21}$ Stadt Offenbach am Main (1998b, 5, Fußnote 3) 


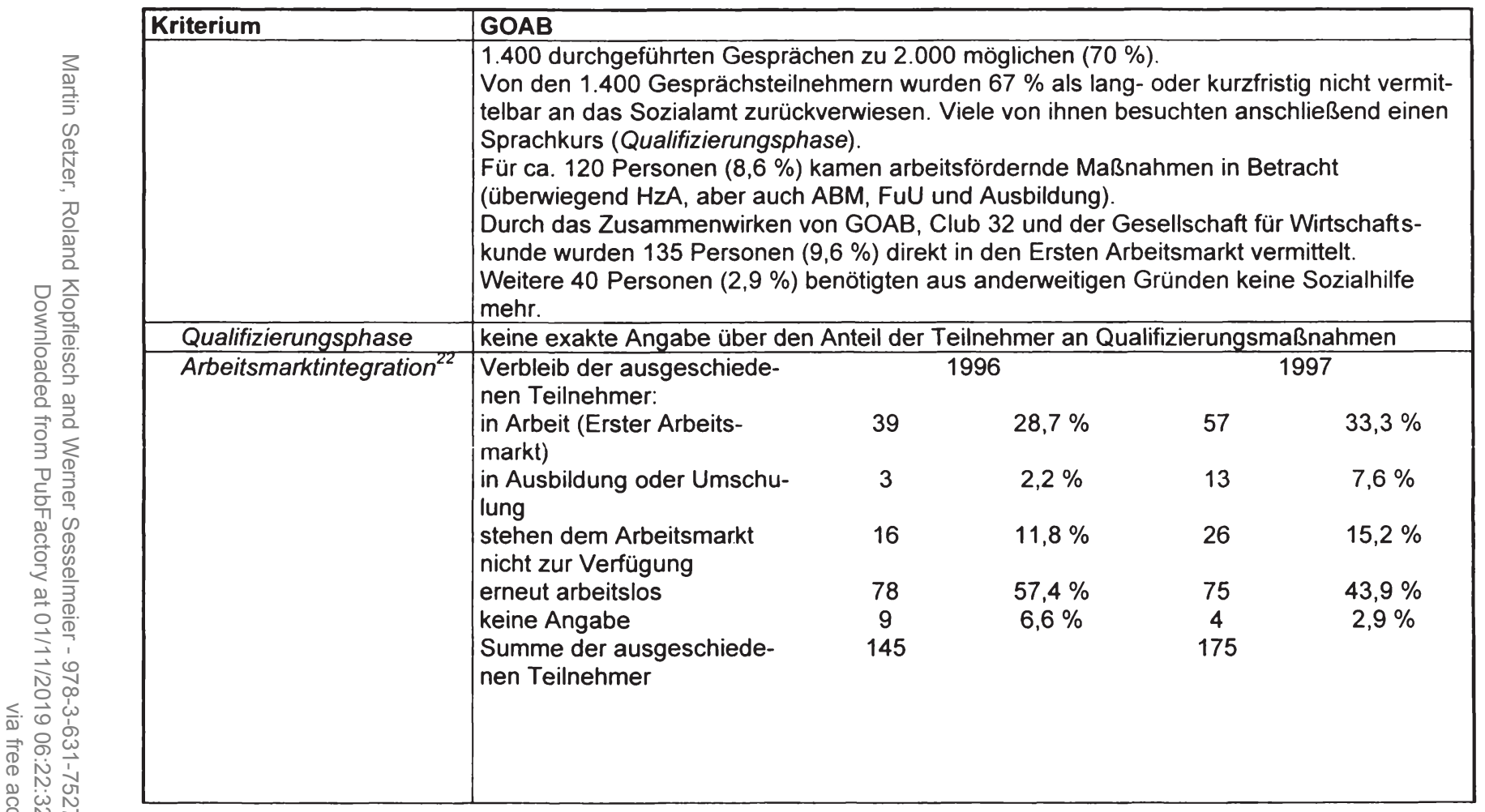

${ }^{22}$ Vgl.: Stadt Offenbach am Main (1998b, 16) 


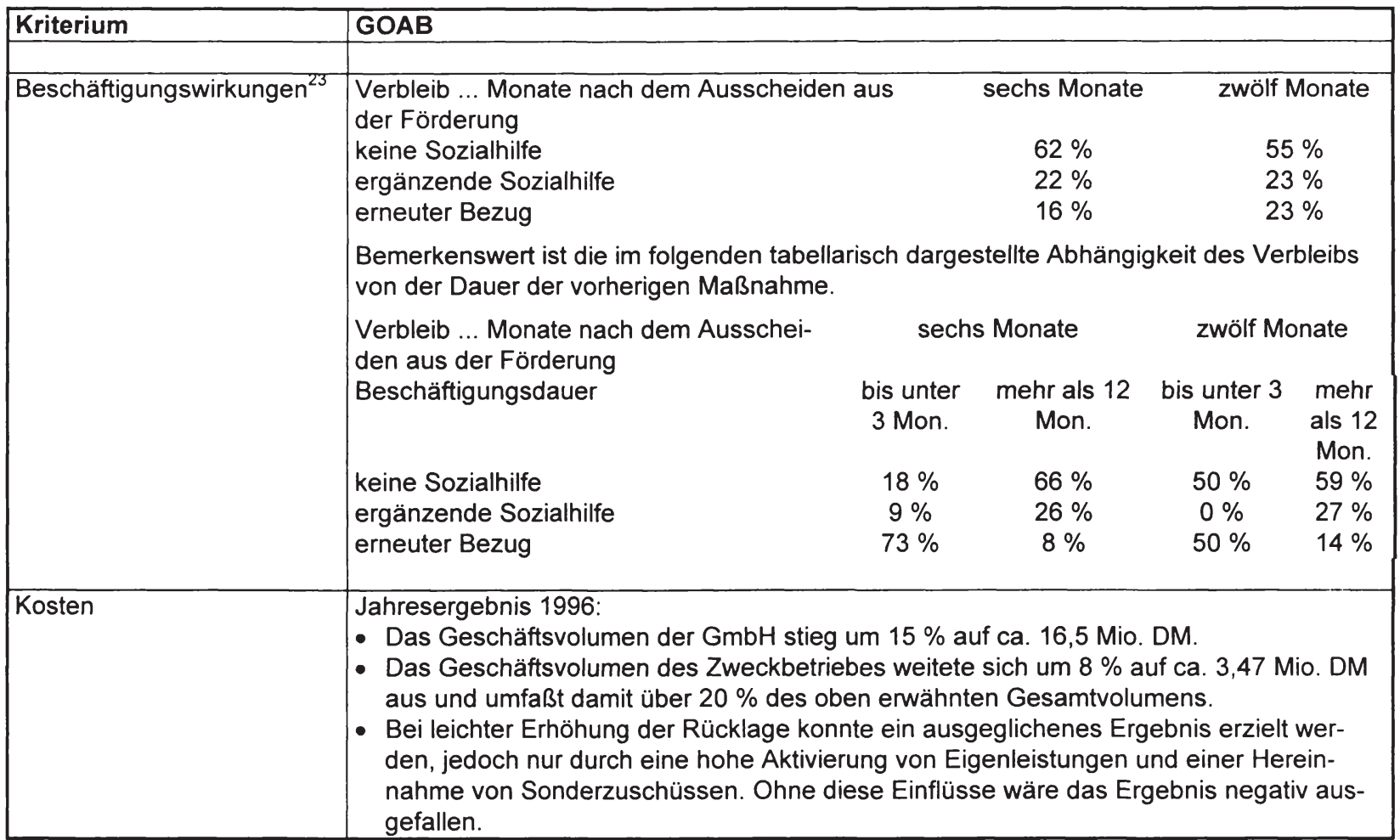

${ }^{23} \mathrm{Vgl}$.: Stadt Offenbach am Main $(1998 b, 21)$ 


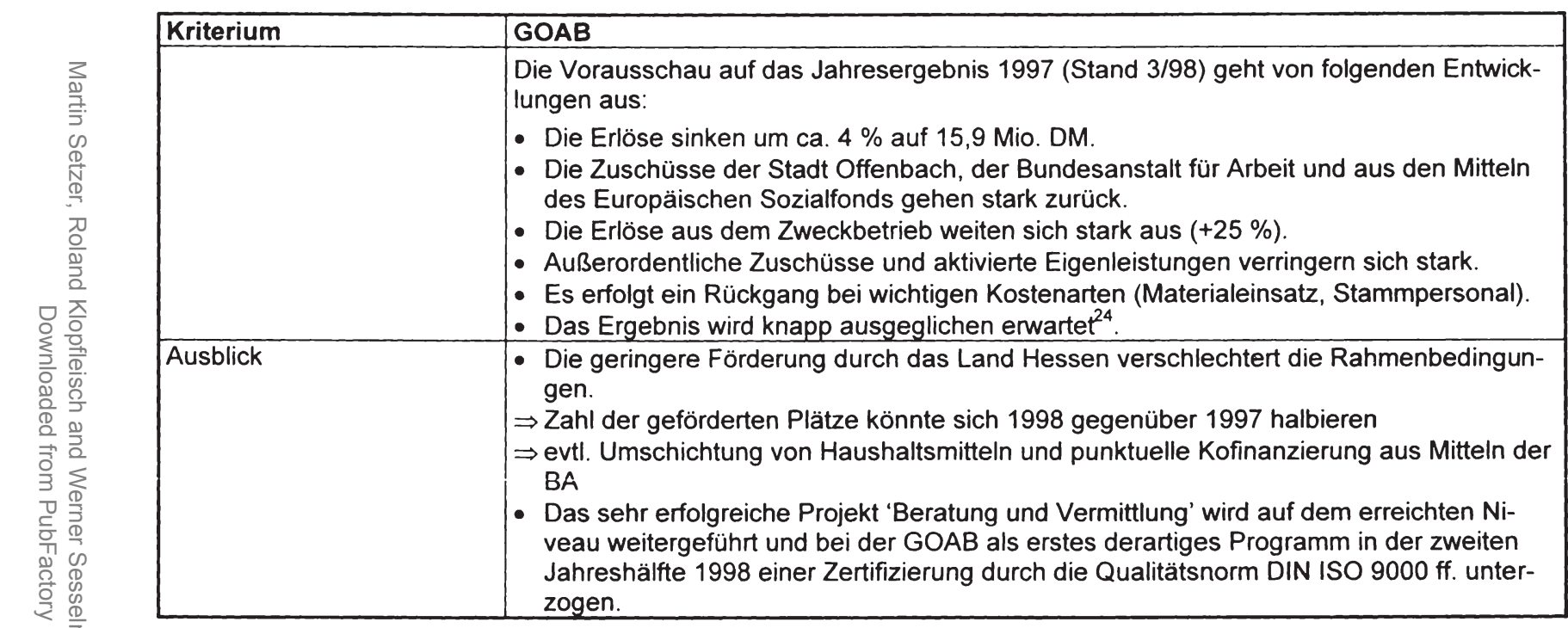

${ }^{24}$ Vgl.: Gemeinnützige Offenbacher Ausbildungs- und Beschäftigungs mbH $(1998,4)$ 


\begin{tabular}{|c|c|}
\hline Kriterium & Fallmanagement Sozialamt Offenbach \\
\hline \multicolumn{2}{|l|}{ Projektstrukturdaten } \\
\hline Trägerschaft & Stadt Offenbach \\
\hline Rechtsform & Abteilung innerhalb des Sozialdezernates (Dienst- und Fachaufsicht) \\
\hline Finanzierungsquellen & Stadt Offenbach \\
\hline Personal & $\begin{array}{l}\text { - ein Referatsleiter (wurde im ersten Projektjahr vom 'bureau maatwerk' gestellt) } \\
\text { - vier Fachkräfte, je zur Hälfte Sozialpädagogen und Sozialvenwalter }\end{array}$ \\
\hline Größe & $\begin{array}{l}\text { Einrichtung im November 1996; bis November } 1997153 \text { Teilnehmer, davon } 124 \text { mit dem } \\
\text { Ziel der Arbeitsvermittlung }\end{array}$ \\
\hline Aufgabenfelder & $\begin{array}{l}\text { Das Referat übernimmt alle Aufgaben und Kompetenzen eines Sachgebietes des Sozial- } \\
\text { amtes und im Einzelfall auch die des Jugendamtes. Besonderes Gewicht wird auf das An- } \\
\text { regen der vorhandenen Selbsthilfepotentiale der Sozialhilfeempfänger gelegt. Sofern not- } \\
\text { wendig, kooperiert das Referat mit folgenden Institutionen: } \\
\text { - Kindertagesstätten (Eigenbetrieb und freie Träger) } \\
\text { - Allgemeiner Sozialer Dienst (bei Erziehungsproblemen) } \\
\text { - Beratungsstellen (bei persönlichen Problemen) } \\
\text { - Schuldnerberatung } \\
\text { - Qualifizierungsträger } \\
\text { - Wohnungsamt und Baugesellschaften (bei Wohnungsproblemen) } \\
\text { - Arbeitsvermittlungsträger }\end{array}$ \\
\hline \multicolumn{2}{|l|}{ Angebote } \\
\hline Dauer der Beschäftigung & $\begin{array}{l}\text { Die Verweilzeiten von durch das Referat Fallmanagement betreuten Arbeitsuchenden in } \\
\text { der Sozialhilfe betragen zwischen drei und sechs Monaten. }\end{array}$ \\
\hline Entlohnung & Sozialhilfe \\
\hline Teilzeit Vollzeit & - \\
\hline Teilnehmergewinnung & $\begin{array}{l}\text { Die Sachbearbeiter der einzelnen Sachgebiete im Sozialamt verweisen solche Fälle an } \\
\text { das Referat Fallmanagement, von denen sie glauben, daß sie nach der Beseitigung be- } \\
\text { stimmter Vermittlungshemmnisse aus der Sozialhilfe ausscheiden könnten. Die Beseiti- } \\
\text { gung dieser Vermittlungshemmnisse bedarf in der Regel einer eingehenden Beschäfti- }\end{array}$ \\
\hline
\end{tabular}




\begin{tabular}{|c|c|c|}
\hline \multirow{3}{*}{\multicolumn{2}{|c|}{ 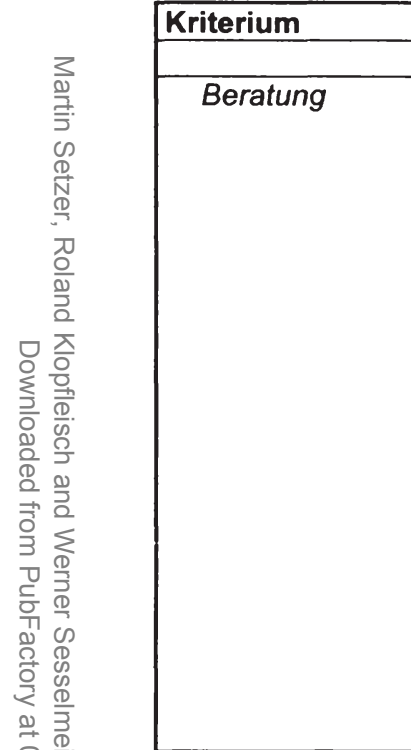 }} & Fallmanagement Sozialamt Offenbach \\
\hline & & gung, für die im Tagesgeschäft der Sachbearbeiter keine Zeit vorhanden ist. \\
\hline & & $\begin{array}{l}\text { Im Rahmen eines Erstberatungsgesprächs erläutern Sozialarbeiter und Sozialhilfesach- } \\
\text { bearbeiter dem Sozialhilfebeziehenden bzw. der vollständigen Bedarfsgemeinschaft } \\
\text { (Familie) das zweifache Projektziel25: } \\
\text { 1. Verbesserung der Lebensqualität durch wirtschaftliche Unabhängigkeit } \\
\text { (Klienteninteresse) } \\
\text { 2. Einsparung von Sozialhilfemitteln (Institutioneninteresse) } \\
\text { In einer Diskussion wird gemeinsam das Ziel konkretisiert. Unterstützend wirkt hierbei das } \\
\text { Ergebnis einer Ist-Analyse der Situation des Sozialhilfebeziehenden. Die Ist-Analyse bein- } \\
\text { haltet sowohl folgende, den Erwerbsprozeß betreffende Punkte: } \\
\text { - Ausbildungs- und Arbeitsbiographie } \\
\text { - erwerbsfördernde und erwerbshemmende Faktoren (Qualifikation, Arbeitserfahrung, } \\
\text { gesundheitliche Einschränkungen) } \\
\text { als auch alle relevanten Informationen zur Lebenslage des Sozialhilfebeziehenden, wie } \\
\text { z.B.: } \\
\text { - die innerfamiliäre Situation } \\
\text { - die vorhandenen formellen und informellen Unterstützungssysteme } \\
\text { - die Kinderbetreuungssituation } \\
\text { - die wirtschaftliche Situation } \\
\text { - die Wohnungssituation }\end{array}$ \\
\hline & Orientierung & Orientierungshilfen sind Bestandteil der Beratungsgespräche (siehe: Beratung). \\
\hline & Qualifizierung & $\begin{array}{l}\text { Das Referat Fallmanagement kooperiert mit Qualifizierungsträgern, um bei Bedarf Sozi- } \\
\text { alhilfeempfänger in eine Qualifizierungsmaßnahmen zu vermitteln (bspw. Deutschkurs, } \\
\text { Computerkurs, Erwerb des Hauptschulabschlusses). }\end{array}$ \\
\hline & Beschäftigung & $\begin{array}{l}\text { Das Referat Fallmanagement ist kein Beschäftigungsträger und vermittelt auch nicht in } \\
\text { solche. Ob und welche Beschäftigung einem Sozialhilfeempfänger angeboten wird, liegt }\end{array}$ \\
\hline
\end{tabular}

${ }^{25} \mathrm{Vgl}$ :: Stadt Offenbach am Main (1998a, 9) 


\begin{tabular}{|c|c|c|c|c|}
\hline Kriterium & \multicolumn{4}{|c|}{ Fallmanagement Sozialamt Offenbach } \\
\hline & \multicolumn{4}{|c|}{ im Verantwortungsbereich der Arbeitsvermittlung (GOAB). } \\
\hline Vermittlung & \multicolumn{4}{|c|}{$\begin{array}{l}\text { Der Sozialhilfeempfänger wird nach Beseitigung aller vermittlungshemmender Faktoren } \\
\text { an eine Arbeitsvermittlungsinstitution (GOAB) weitergeleitet. }\end{array}$} \\
\hline Betreuung & \multicolumn{4}{|c|}{$\begin{array}{l}\text { Durch die enge Zusammenarbeit mit den kooperierenden Stellen ist eine kontinuierliche } \\
\text { Betreuung der Sozialhilfeempfänger bis zum Ausscheiden aus dem Fallmanagement } \\
\text { möglich. Eine Betreuung nach Arbeitsaufnahme auf dem Ersten Arbeitsmarkt liegt hinge- } \\
\text { gen im Verantwortungsbereich der vermittelnden Stelle (GOAB). }\end{array}$} \\
\hline Instrumente & \multicolumn{4}{|c|}{$\begin{array}{l}\text { Nach Beseitigung der vermittlungshemmenden Merkmale findet der Arbeitssuchende ent- } \\
\text { weder selbständig oder über eine kooperierende Arbeitsvermittlung (GOAB) eine Stelle. }\end{array}$} \\
\hline \multicolumn{5}{|l|}{ Erfolgskriterien } \\
\hline Erreichen der Zielgruppe & \multicolumn{4}{|l|}{ ausschließlich Sozialhilfeempfänger } \\
\hline \multicolumn{5}{|l|}{ Integrationsleistungen } \\
\hline Projektintegration & \multicolumn{4}{|l|}{ siehe: Arbeitsmarktintegration } \\
\hline Qualifizierungsphase & \multicolumn{4}{|l|}{ siehe: Arbeitsmarktintegration } \\
\hline Arbeitsmarktintegration & $\begin{array}{l}\text { in Arbeit vermittelt } \\
\text { in Qualifizierungsmaßnahmen } \\
\text { in Arbeitsvermittlung bei anderen } \\
\text { in Bearbeitung von FAMS } \\
\text { als nicht vermittelbar eingestuft } \\
\text { aus anderen Gründen aus FAMS } \\
\text { total }\end{array}$ & $\begin{array}{r}28 \\
2 \\
24 \\
44 \\
13 \\
13 \\
124\end{array}$ & $\begin{array}{r}23 \% \\
2 \% \\
19 \% \\
35 \% \\
10 \% \\
10 \% \\
100 \% \\
\end{array}$ & $\begin{array}{l}\text { Von den } 28 \text { in den Arbeitsmarkt } \\
\text { eigener Anstrengung einen } \\
\text { Arbeitsmarkt erhalten. }\end{array}$ \\
\hline Beschäftigungswirkungen & \multicolumn{4}{|l|}{ Keine Angaben } \\
\hline
\end{tabular}




\begin{tabular}{|l|l|}
\hline Kriterium & Fallmanagement Sozialamt Offenbach \\
\hline & \\
\hline Kosten & "Im April 98 stehen entsprechend nach dieser Bemessung ca. 300.000 DM an zusätzli- \\
& chen Personalkosten einer Verringerung des Sozialhilfebedarfs zwischen 400.000 und \\
& 1,1 Mio. DM gegenüber"26 \\
\hline
\end{tabular}

\footnotetext{
${ }^{26}$ Stadt Offenbach am Main $(1998 a, 32)$
} 


\begin{tabular}{|c|c|}
\hline Kriterium & Selbsthilfe im Taunus e.V. (SiT e.V.) \\
\hline \multicolumn{2}{|l|}{ Projektstrukturdaten } \\
\hline Trägerschaft & freie Trägerschaft \\
\hline Rechtsform & gemeinnütziger eingetragener Verein \\
\hline Finanzierungsquellen & $\begin{array}{l}\text { "Aufgrund der Beschränkungen im Bereich der öffentlichen Zuwendungen ist die SiT ge- } \\
\text { zwungen, aus verschiedenen Bereichen Gelder zu beantragen,27. Diese sind im einzel- } \\
\text { nen: } \\
\text { - Land Hessen } \\
\text { - Arbeitsamt } \\
\text { - Main-Taunus-Kreis } \\
\text { - Städte, in denen die jeweiligen Projekte durchgeführt werden } \\
\text { - veschiedene Ministerien } \\
\text { - EU (projektbezogene Förderung) } \\
\text { - zu einem Drittel Eigenfinanzierung }\end{array}$ \\
\hline Personal & $\begin{array}{l}\text { - } 37 \text { Stammkräfte in den Bereichen Geschäftsstelle, Personal- und Finanzwesen, Anlei- } \\
\text { tung im Arbeitsbereich sowie im Beratungsangebot } \\
\text { - pro Jahr durchschnittlich } 60 \text { Praktikanten in 4- bis 6-monatiger Arbeitserporbung } \\
\text { - In der Clearing-Stelle arbeiteten } 1997 \text { eine Voll- und eine Halbtagskraft; seit kurzem ist } \\
\text { eine weitere Vollzeitkraft eingestellt. } \\
\text { - Die Vermittlungsarbeit von WORK-IN lastet auf den Schultern von drei Personaldispo- } \\
\text { nenten. }\end{array}$ \\
\hline Größe & $\begin{array}{l}\text { - Die Clearing-Stelle führte im ersten Halbjahr } 1998 \text { mit } 500 \text { Sozialhilfeempfängern Erst- } \\
\text { gespräche durch. } \\
\text { - Innerhalb der SiT-eigenen Betriebe arbeiteten } 1997 \mathrm{ca} .240 \text { Mitarbeiter in ABM- oder } \\
\text { HzA-Maßnahmen. Der Anteil an Sozialhilfeempfängern beträgt } 1998 \mathrm{ca} .70 \%{ }^{28} \text {. } \\
\text { - WORK-IN beschäftigte } 199782 \text { Mitarbeiter - darunter } 22 \text { Sozialhilfeempfänger - per }\end{array}$ \\
\hline
\end{tabular}

${ }^{27}$ SiT-Selbsthilfe im Taunus $(1997,2)$

${ }^{28}$ Stand Oktober 1998 


\begin{tabular}{|c|c|}
\hline Kriterium & Selbsthilfe im Taunus e.V. (SiT e.V.) \\
\hline & $\begin{array}{l}\text { Uberlassungsvertrag im Ersten Arbeitsmarkt }{ }^{29} \text { (1998: } 86 \text { Personen, darunter } \\
40 \text { Sozialhilfeempfänger }{ }^{28} \text { ). } \\
\text { - Im Rahmen des Frauenprojektes "Hauswirtschaftliche Hilfen" existieren acht Voll- } \\
\text { zeit- und vier Halbtagsstellen. } 1997 \text { wurden mit } 43 \text { interessierten Frauen Beratungsge- } \\
\text { spräche druchgeführt }{ }^{30} \text {. }\end{array}$ \\
\hline Aufgabenfelder & $\begin{array}{l}\text { Die SiT unterhält } 23 \text { Zweckbetriebe bzw. Projekte. } \\
\text { Im Vermittlungsbereich existiert ein Netz aus folgenden Instanzen: } \\
\text { - Clearing-Stelle: } \\
\text { vorgeschaltete Instanz bei der Vermittlung von Sozialhilfeempfängern }{ }^{31} \\
\text { - Fachstelle "Berufliche Begleitung und Vermittlungsvorbereitung": } \\
\text { Betreuung der in den Beschäftigungsbetrieben der SiT beschäftigten Personen }{ }^{32} \\
\text { - WORK-IN } \\
\text { ist die gemeinnützige Arbeitnehmerüberlassung und Personalvermittlung der SiT. Sie } \\
\text { vermittelt in folgende Bereiche des Ersten Arbeitsmarktes: } \\
\text { - gewerblicher Bereich (Hilfskräfte) } \\
\text { - medizinscher Bereich: } \\
\text { - ausgebildete MTA }\end{array}$ \\
\hline
\end{tabular}

${ }^{29}$ Vgl.: SiT-Selbsthilfe im Taunus $(1997,6 f$.

${ }^{30}$ Vgl.: SiT-Selbsthilfe im Taunus $(1997,7 f$.

31 "Die Clearing-Stelle vermittelt nach intesiven Beratungsgesprächen und sorgfältiger Prüfung von Motivation und Fähigkeiten Sozialhilfeempfägner an die "richtige Stelle" weiter. Dies kann die Direktvermittlung auf den allgemeinen Arbeitsmarkt sein, die Überlassung und Personalvermittlung durch WORK-IN oder die Vermittlung in die Betriebe der SiT. Darüber hinaus besteht die Möglichkeit einer zeitlich begrenzten Arbeitserprobung in den SiT-Betrieben" (SiT-Selbsthilfe im Taunus 1997, 2).

32 Die Stelle 'Interne berufliche Beratung und Vermittlungsorientierung' informiert Maßnahmeteilnehmer bereits während der Maßnahme über berufliche Perspektiven und bereitet diese mit Hilfe eines gemeinsam entwickelten Eingliederungsplans auf die Vermittlung auf den allgemeinen bzw. auf mögliche Umschulungs- und Weiterbildungsmaßnahmen vor. 


\begin{tabular}{|c|c|}
\hline Kriterium & Selbsthilfe im Taunus e.V. (SiT e.V.) \\
\hline & $\begin{array}{l}\text { - Krankengymnasten } \\
\text { - Altenpfleger } \\
\text { - examinierte Krankenschwestern und -pfleger } \\
\text { - Ausdehnung auf den kaufmännischen Bereich aufgrund der großen Nachfrage } \\
\text { - Frauenprojekt }\end{array}$ \\
\hline \multicolumn{2}{|l|}{ Angebote } \\
\hline Dauer der Beschäftigung & $\begin{array}{l}\text { Sowohl die Arbeitsverträge im Rahmen von WORK-IN als auch die der ABM-oder } \\
\text { HzA-Maßnahmen in den SiT-Betrieben sind auf die Dauer eines Jahres befristet. Eine } \\
\text { einmalige Verlängerung um ein weiteres Jahr ist möglich. }\end{array}$ \\
\hline Entlohnung & Die Entlohnung aller Arbeitsverträge ist tariflich. \\
\hline TeilzeitNollzeit & ABM- und HzA-Stellen existieren sowohl als Vollzeit- als auch als Teilzeitstellen. \\
\hline \multirow[t]{3}{*}{ Teilnehmergewinung } & $\begin{array}{l}\text { Clearing-Stelle: } \\
\text { - Die Clearing-Stelle ist eine Anlaufstelle ausschließlich für Sozialhilfeempfänger. Im glei- } \\
\text { chen Gebäude wie das Sozialamt angesiedelt, führen die Vermittlungskräfte vornehm- } \\
\text { lich mit Erstantragstellern Beratungsgespräche durch, um die Verweilzeiten in der So- } \\
\text { zialhilfe so kurz wie möglich zu halten }{ }^{33} \text {. }\end{array}$ \\
\hline & $\begin{array}{l}\text { SiT-interne Fachstelle "Berufliche Begleitung und Vermittlungsvorbereitung": } \\
\text { - Die Zielgruppe der Vermittlungstätigkeit dieser internen Fachstelle sind ausschließlich } \\
\text { die Mitarbeiter in den SiT-Betrieben. Eine frühestmögliche Vermittlung in den Ersten } \\
\text { Arbeitsmarkt wird angestrebt. Die in den internen Betrieben beschäftigten Personen } \\
\text { gelangen entweder über das Arbeitsamt, das Sozialamt oder durch Eigeninitiative zur } \\
\text { SiT. }\end{array}$ \\
\hline & $\begin{array}{l}\text { WORK-IN: } \\
\text { - Das Klientel von WORK-IN gelangt entweder über die Clearing-Stelle, über die } \\
\text { SiT-interne Fachstelle "Berufliche Begleitung und Vermittlungsvorbereitung" oder durch } \\
\text { Eigeninitiative zu WORK-IN. }\end{array}$ \\
\hline
\end{tabular}

${ }^{33}$ Vgl.: SiT-Selbsthilfe im Taunus $(1997,5)$ 


\begin{tabular}{|c|c|c|}
\hline \multirow{2}{*}{\multicolumn{2}{|c|}{\begin{tabular}{l|l} 
& Kriterium \\
\cline { 2 - 2 } & Beratung \\
&
\end{tabular}}} & Selbsthilfe im Taunus e.V. (SiT e.V.) \\
\hline & & $\begin{array}{l}\text { - Beratungsgespräche zur Vermittlung in den Ersten Arbeitsmarkt (Clearing-Stelle, } \\
\text { SiT-interne Fachstelle "Berufliche Begleitung und Vermittlungsvorbereitung") } \\
\text { - Suchtberatung (ursprüngliches Aktionsfeld der SiT) } \\
\text { - Schuldnerberatung } \\
\text { - Beratungsstelle für Jugendliche im Übergang von Schule und Beruf (JUBB) })^{34}\end{array}$ \\
\hline & Orientierung & $\begin{array}{l}\text { Orientierungshilfen sind Bestandteil der Beratungsgespräche der Clearing-Stelle und der } \\
\text { SiT-internen Fachstelle "Berufliche Begleitung und Vermittlungsvorbereitung". Darüber } \\
\text { hinaus organisiert die SiT einwöchige "Bewerbungs- und Strategieseminare" für die Per- } \\
\text { sonen, für die eine eingehendere Orientierung sinnvoll erscheint. }\end{array}$ \\
\hline$\sum_{\substack{0 \\
0} \frac{0}{0}}^{\frac{0}{0}}$ & Qualifizierung & $\begin{array}{l}\text { Qualifizierungsmaßnahmen werden sowohl intern in den Beschäftigungsbetrieben (z.B. } \\
\text { Gabelstaplerschein) als auch bei externen Bildungsträgern (z.B. Sprachkurse) durchge- } \\
\text { führt. }\end{array}$ \\
\hline 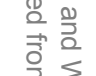 & Beschäftigung & $\begin{array}{l}\text { Die SiT beschäftigt Arbeitslose in insgesamt } 23 \text { Zweckbetrieben und Projekten (ABM- und } \\
\text { HzA-Maßnahmen). }\end{array}$ \\
\hline 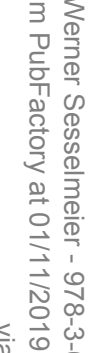 & Vermittlung & 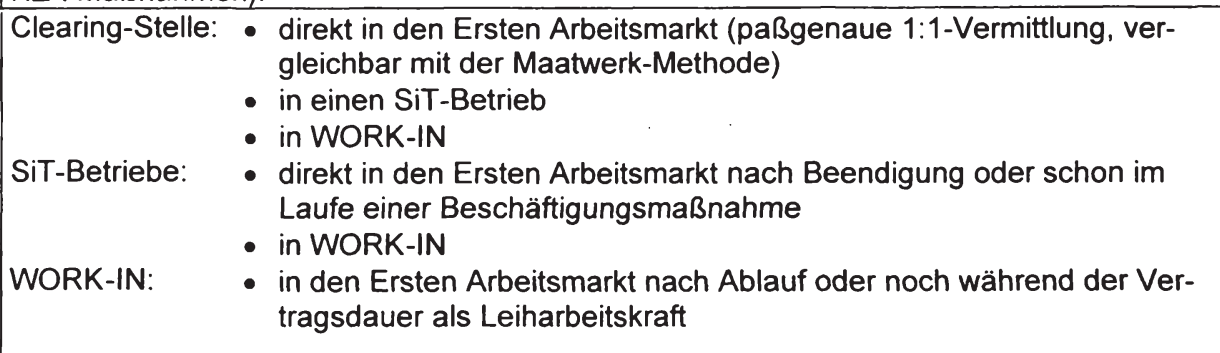 \\
\hline
\end{tabular}

${ }^{34}$ kooperiert mit der neu eingerichteten Koordinierungsstelle "Jugendberufshilfe", welche in Zusammenarbeit mit dem Kreis und anderen relevanten Stellen Möglichkeiten zur Ausbildung von benachteiligten Jugendlichen konzipieren, entwickeln und umsetzen soll (Vgl.: SiT-Selbsthilfe im Taunus 1997, 2) 


\begin{tabular}{|c|c|}
\hline Kriterium & Selbsthilfe im Taunus e.V. (SiT e.V.) \\
\hline Betreuung & $\begin{array}{l}\text { Auch im Anschluß an eine Vermittlung in den Ersten Arbeitsmarkt steht den ehemaligen } \\
\text { Maßnahmeteilnehmern der Weg zu weiteren Beratungsgesprächen offen. Diese weiter- } \\
\text { führende Betreuung findet auf freiwilliger Basis statt. }\end{array}$ \\
\hline Instrumente & $\begin{array}{l}\text { Die SiT nutzt zu Vermittlungszwecken alle Formen gleitender Übergänge. } \\
\text { - Praktika } \\
\text { - gemeinnützige Arbeit } \\
\text { - Beschäftigung in den SiT-Betrieben } \\
\text { - Arbeitnehmerüberlassung }\end{array}$ \\
\hline \multicolumn{2}{|l|}{ Erfolgskriterien } \\
\hline Erreichen der Zielgruppe & $\begin{array}{ll}\text { Clearing-Stelle: } & \text { ausschließlich Sozialhilfeempfänger (500 Erstberatungsgespräche im } \\
& \text { ersten Halbjahr 1998) } \\
\text { SiT-Betriebe: } & 240 \text { Mitarbeiter im Jahr 1997, Anteil der Sozialhilfeempfänger beträgt ca. } \\
\text { WORK-IN: } & 70 \% \\
& 1997: 22 \text { Sozialhilfeempfänger unter } 82 \text { per Überlassung Beschäftigten } \\
& (26,8 \%) \\
& 1998: 40 \text { Sozialhilfeempfänger unter } 86 \text { per Überlassung Beschäftigten } \\
& (46,5 \%)\end{array}$ \\
\hline \multicolumn{2}{|l|}{ Integrationsleistungen } \\
\hline Projektintegration & $\begin{array}{l}\text { Es existieren keine Angaben darüber, wieviele der } 500 \text { Sozialhilfeempfänger, mit denen } \\
\text { seit Beginn } 1998 \text { Erstberatungsgespräche durchgeführt wurden, Aufnahme in die einzel- } \\
\text { nen Projekte und/oder Maßnahmen fanden. }\end{array}$ \\
\hline Qualifizierungsphase & siehe Projektintegration \\
\hline Arbeitsmarktintegration & $\begin{array}{l}\text { Die Zahlen in der nachfolgenden Tabelle beziehen sich auf das Jahr } 1997 \text { und die Vermitt- } \\
\text { lungstätigkeit von WORK-IN sowie der SiT-internen Fachstelle "Berufliche Begleitung und } \\
\text { Vermittlungsvorbereitung". Die Clearing-Stelle arbeitet in ihrer heutigen Form erst seit Be- } \\
\text { ginn des Jahres } 1998 \text {. }\end{array}$ \\
\hline
\end{tabular}




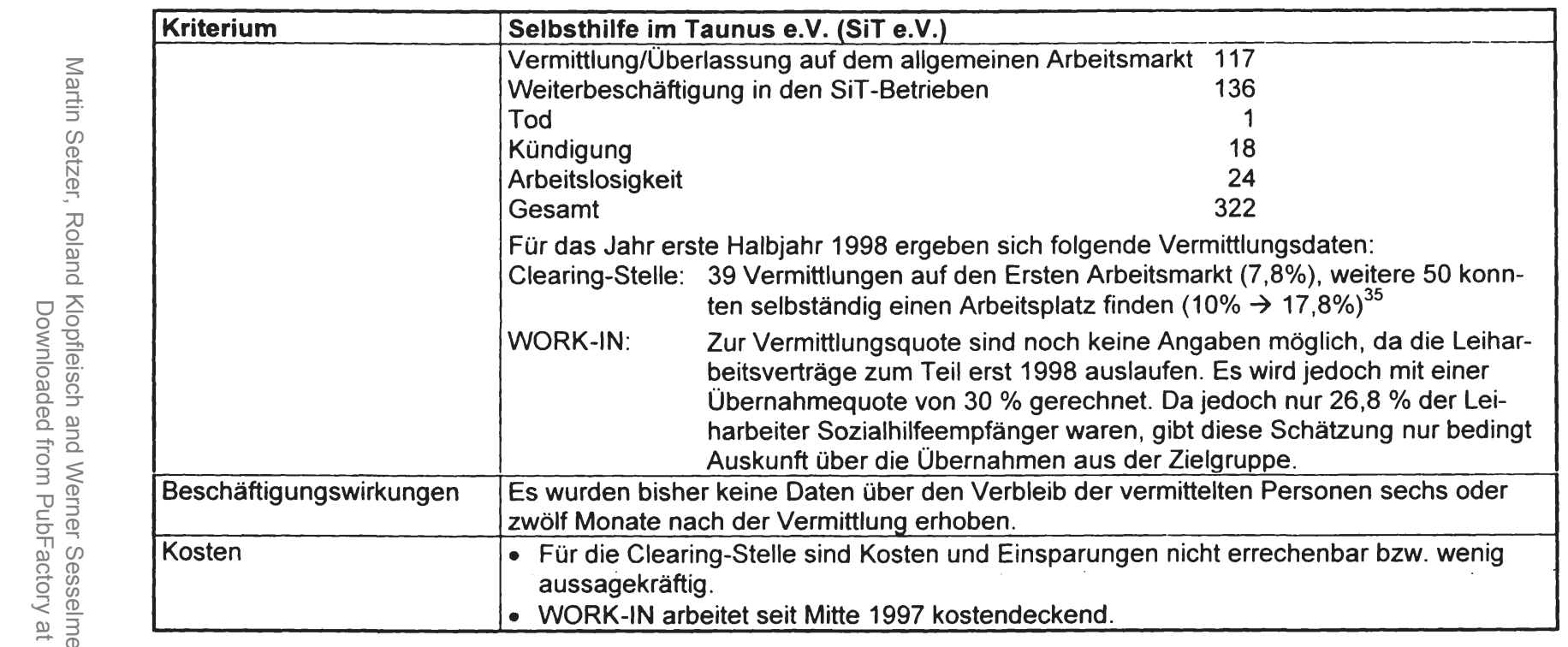

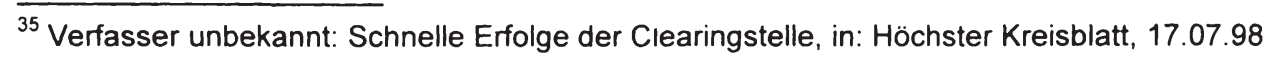




\begin{tabular}{|c|c|}
\hline Kriterium & START Zeitarbeit NRW GmbH \\
\hline \multicolumn{2}{|l|}{ Projektstrukturdaten } \\
\hline Trägerschaft & seit Dezember 1997 in freier Trägerschaft \\
\hline Rechtsform & $\mathrm{GmbH}$ \\
\hline Finanzierungsquellen & $\begin{array}{l}\text { - Seit Dezember } 1997 \text { wirtschaftlich eigenständig handelnd } \\
\text { - Leihgebühren für die entliehenen Arbeitskräfte (in der Regel das Doppelte des Brutto- } \\
\text { lohns ohne Sozialversicherungsbeiträge) } \\
\text { - individuelle Förderleistungen, wie bspw. Lohnkostenzuschüsse }\end{array}$ \\
\hline Personal & Stammbelegschaft: 141 Personen (davon 30 in der Hauptverwaltung) \\
\hline Größe & In den Jahren 1995 bis 1997 wurden insgesamt 5.851 Leiharbeitskräfte neu eingestellt. \\
\hline Aufgabenfelder & $\begin{array}{l}\text { START verleiht seine Leiharbeitskräfte vornehmlich in Unternehmen des produzierenden } \\
\text { Gewerbes (Metall- und Elektrobereich). Der Dienstleistungssektor ist vor allem im kauf- } \\
\text { männischen Bereich deutlich unterrepräsentiert. Alle Leiharbeitskräfte erhalten von } \\
\text { START einen Arbeitsvertrag. Ihre Einsatzbereiche befinden sich ausschließlich auf dem } \\
\text { Ersten Arbeitsmarkt. }\end{array}$ \\
\hline \multicolumn{2}{|r|}{ 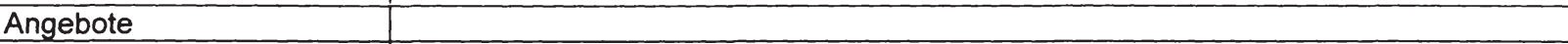 } \\
\hline Dauer der Beschäftigung & $\begin{array}{l}\text { - unbefristete Arbeitsverträge mit START } \\
\text { - "Betriebsbedingte Kündigungen sind zulässig - allerdings erst nach einer angemesse- } \\
\text { nen Weiterbeschäftigungsfrist, die mindestens ein Viertel der Dauer des ersten Ver- } \\
\text { leiheinsatzes ausmachen muß"37. }\end{array}$ \\
\hline Entlohnung & nach dem Tarif des Entleihbetriebs durch START \\
\hline TeilzeitNollzeit & keine Angaben \\
\hline Teilnehmergewinnung & $\begin{array}{l}\text { - überwiegend über die Arbeitsämter } \\
\text { - zunehmend über informelle Kanäle, bspw. Freunde der eingestellten Leiharbeitskräfte } \\
\text { - selten über die Sozialämter (1997 nur } 114 \text { von } 2.166 \text { Neueinstellungen }=5,3 \%)^{38}\end{array}$ \\
\hline
\end{tabular}

${ }^{36}$ Vgl.: Vanselow, AchimMeinkopf, Claudia $(1996,15)$

${ }^{37}$ Vanselow, Achim Weinkopf, Claudia $(1996,20)$

${ }^{38}$ Vgl.: Vanselow, AchimMeinkopf, Claudia $(1998,12)$ 


\begin{tabular}{|c|l|}
\hline Kriterium & START Zeitarbeit NRW GmbH \\
\hline Beratung & Beratungen, bspw. bei Verschuldungsproblemen oder fehlenden Kinderbetreuungsplät- \\
zen, finden im Rahmen von sogenannten Vorqualifizierungsmaßnahmen statt, welche der \\
Einstellung und Arbeitsaufnahme bei START vorgeschaltet sind. \\
Neben Beratungen beinhalten diese Maßnahmen andere sozialqualifikatorische oder auch \\
berufsfachliche Elemente, je nach Bedarf des Arbeitssuchenden. Die Teilnehmer an den \\
Vorqualifizierungsmaßnahmen rekrutieren sich aus Zielgruppenangehörigen ${ }^{39}$, bei denen \\
START zur (Wieder-)herstellung ihrer Arbeitsfähigkeit, insbesondere zur persönlichen \\
Stabilisierung und zur Vorbereitung auf den Einsatz als Leiharbeitskraft, eine solche Schu- \\
lung für notwendig erachtet. Darüber hinaus stehen die Veranstaltungen allen interessier- \\
ten Arbeitslosen offen. \\
$\begin{array}{l}\text { Finanziert werden die Vorqualifizierungsmaßnahmen durch die Bundesanstalt für Arbeit } \\
\text { (\$ 103 b AFG) }\end{array}$ \\
\hline $\begin{array}{l}\text { Orientierungshilfen finden im Rahmen der Vorqualifizierungsmaßnahmen statt (siehe: Be- } \\
\text { ratung). }\end{array}$ \\
$\begin{array}{l}\text { START verpflichtete sich schon im Vorfeld der Pilotphase (1992-1995) zur Qualifizierung } \\
\text { von Leiharbeitskräften in verleihfreien Zeiten unter dem Vorbehalt der Finanzierbarkeit. In } \\
\text { der Pilotphase wurden jedoch keine Qualifizierungsmaßnahmen durchgeführt. Dies hatte } \\
\text { sowohl finanzielle als auch organisatorische Gründe: } \\
\text { - Während einer Qualifizierungsmaßnahme erhält START keine Einnahmen aus dem } \\
\text { Entleihgeschäft, muß jedoch gleichzeitig die Kosten für die Qualifizierungsmaßnahme } \\
\text { tragen und zudem den Maßnahmeteilnehmern Tariflohn bzW. Unterhaltskosten zahlen. } \\
\text { Der Maßnahmenteilnehmer erhält keine öffentlichen Zuschüsse nach dem } \\
\text { SGB III/AFG, da er bei START unter Vertrag steht. Finanzierungen mittels des } \\
\text { SGB III/AFG sind dementsprechend nur vor einer Neueinstellung möglich. } \\
\text { START unterhält keine eigenen Bildungseinrichtungen. Die beauftragten Bildungsträger }\end{array}$ \\
\hline
\end{tabular}

${ }^{39}$ Die Zielgruppe ist unter dem Punkt Erreichen der Zielgruppe weiter unten definiert.
${ }^{40}$ Vgl.: Vanselow, AchimMeinkopf, Claudia $(1996,24 f$. 


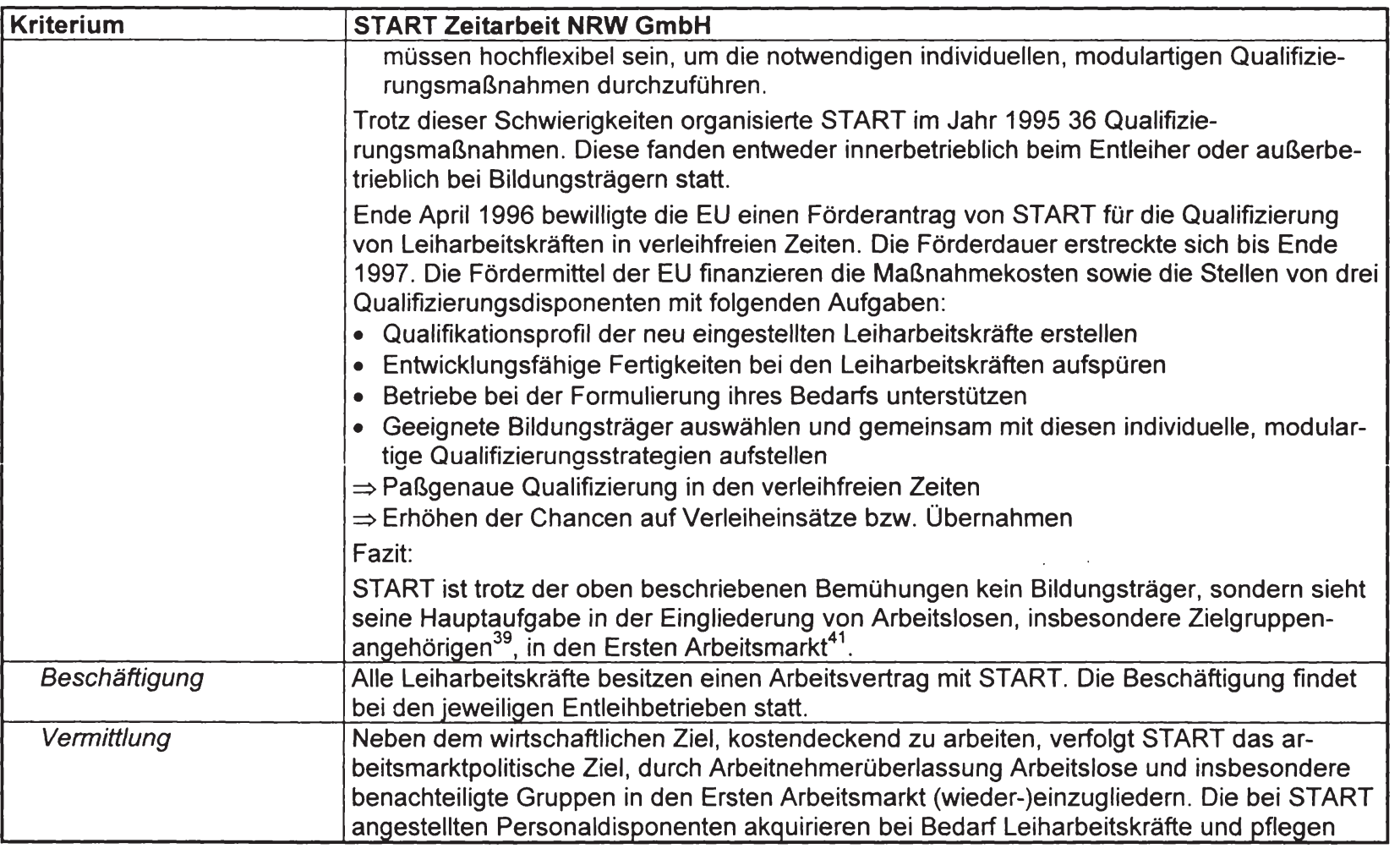

${ }^{41}$ Vgl.: Vanselow, Achim/Weinkopf, Claudia $(1996,20 f f$. 


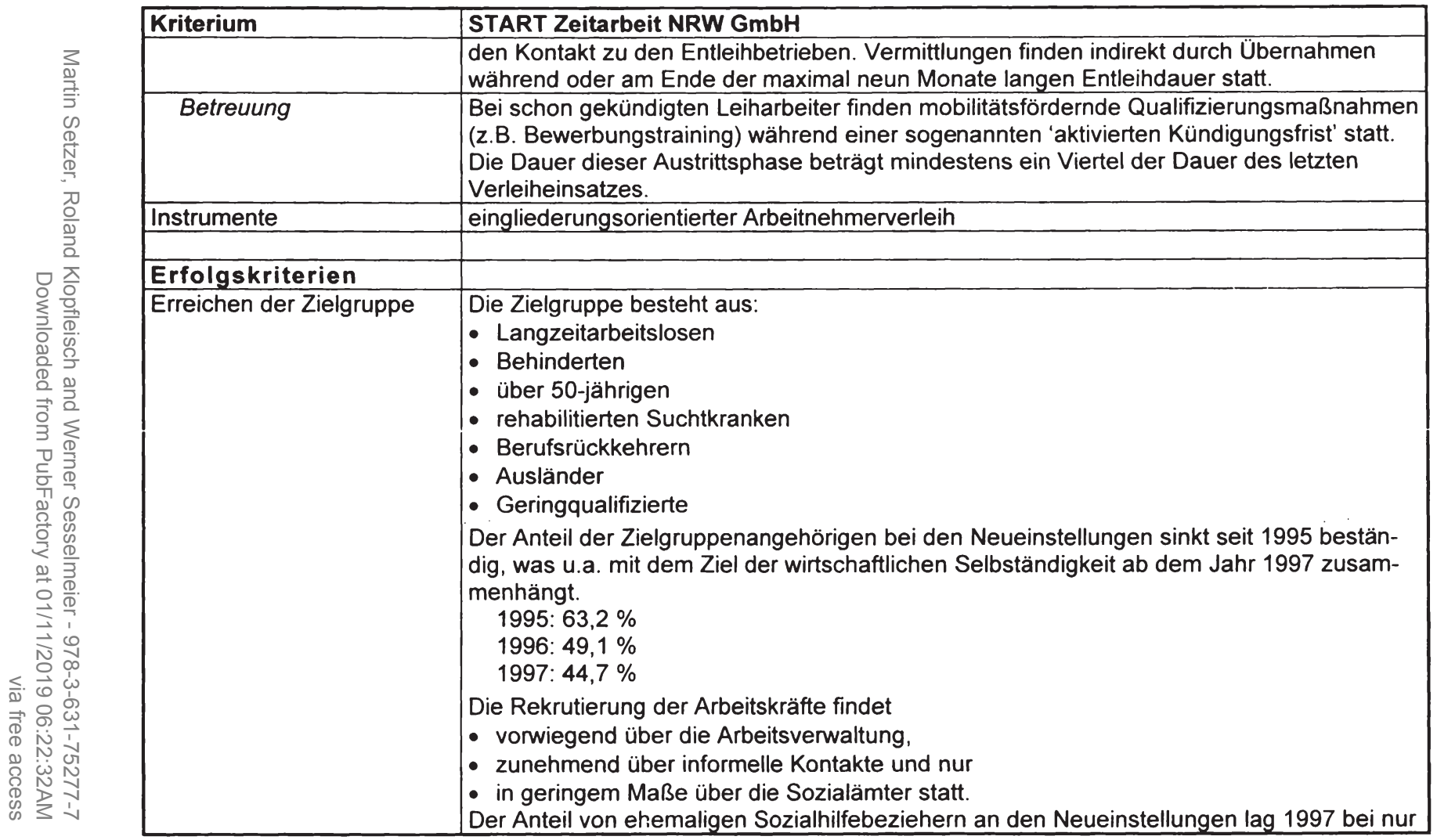




\begin{tabular}{|c|c|c|c|c|}
\hline Kriterium & \multicolumn{4}{|l|}{ START Zeitarbeit NRW GmbH } \\
\hline & \multicolumn{4}{|l|}{$5,3 \%$ (Vgl.: Teilnehmergewinnung). } \\
\hline \multicolumn{5}{|l|}{ Integrationsleistungen } \\
\hline \multirow[t]{2}{*}{ Projektintegration } & \multirow{2}{*}{\multicolumn{4}{|c|}{ 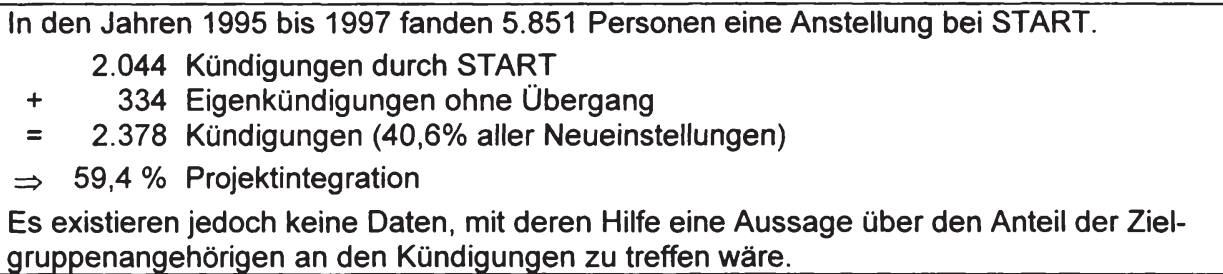 }} \\
\hline & & & & \\
\hline Qualifizierungsphase & \multicolumn{4}{|c|}{ START verpflichtet sich, in verleihfreien Zeiten Qualifizierungsmaßnahmen durchzuführen. } \\
\hline \multirow[t]{7}{*}{ Arbeitsmarktintegration } & \multicolumn{4}{|c|}{$\begin{array}{l}\text { Die Entleihbetriebe übernahmen in den Jahren } 1995 \text { bis } 1997 \text { insgesamt } \\
\text { 1.917 Leiharbeitskräfte. Dies sind: } \\
32,8 \% \text { aller Neueinstellungen bzw. } \\
44,6 \% \text { aller bis zuim Jahresende } 1997 \text { Ausgeschiedenen }\end{array}$} \\
\hline & \multicolumn{4}{|c|}{$\begin{array}{l}\text { Von den } 1.917 \text { Arbeitskräften zählen } 786 \text { zu der Zielgruppe. } \\
\Rightarrow 41,0 \% \text { Arbeitsmarktintegration der Zielgruppenangehörigen }\end{array}$} \\
\hline & \multicolumn{4}{|c|}{$\begin{array}{l}\text { Der Anteil der Zielgruppenangehörigen an den Übernahmen schwankte von Jahr zu Jahr. } \\
\text { Die folgende Tabelle gibt Aufschluß über die jährlichen Übernahmequoten von Zielgrup- } \\
\text { penangehörigen in feste Beschäftigungsverhältnisse des Ersten Arbeitsmarkts }{ }^{42} \text {. }\end{array}$} \\
\hline & $\begin{array}{l}\text { Übernahmequoten von Zielgruppenangehörigen } \\
\text { davon: }\end{array}$ & $\begin{array}{c}1995 \\
28,5 \%\end{array}$ & $\begin{array}{c}1996 \\
49,5 \%\end{array}$ & $\begin{array}{c}1997 \\
39,1 \%\end{array}$ \\
\hline & Langzeitarbeitslose & $48,4 \%$ & $31,0 \%$ & $44,8 \%$ \\
\hline & Ausländer & $18,3 \%$ & $34,8 \%$ & $32,2 \%$ \\
\hline & Geringqualifizierte & $17,2 \%$ & $20,5 \%$ & $11,5 \%$ \\
\hline
\end{tabular}

$\overline{{ }^{42} \text { Vgl.: Vanselow, Achim/Neinkopf, Claudia }(1998,18)}$ 


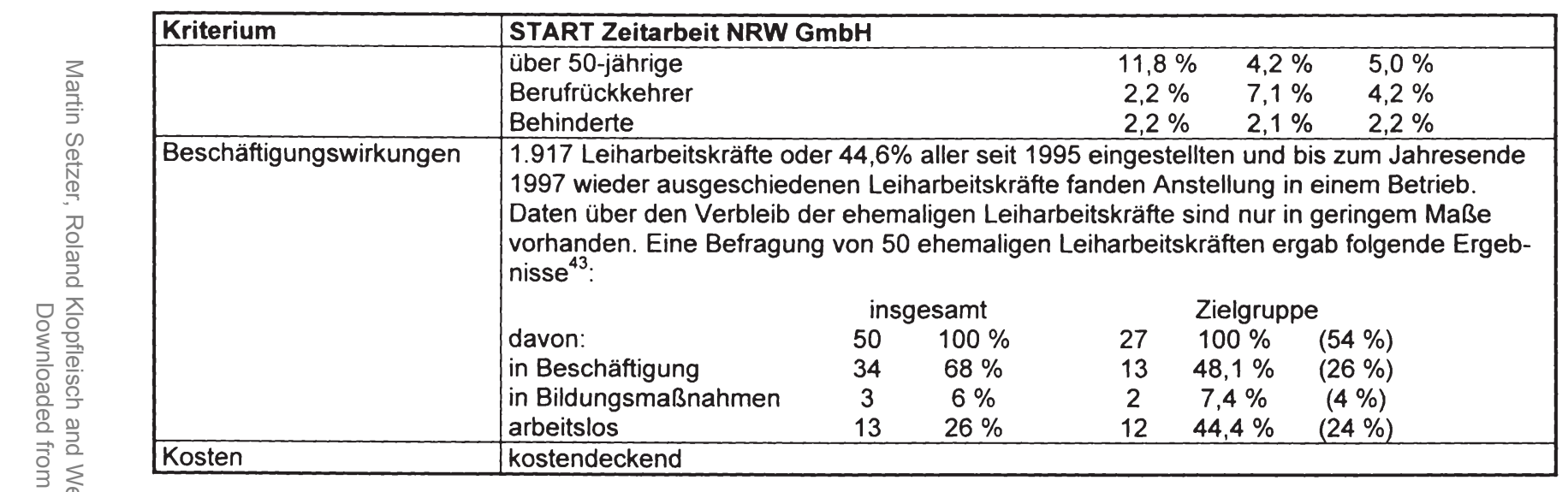

$\overline{{ }^{43} \text { Vgl.: Vanselow, Achim/Weinkopf, Claudia }(1998,32 f f .)}$ 


\begin{tabular}{|l|l|}
\hline Kriterium & Projekt 1.000 Ressourcen \\
\hline Projektstrukturdaten & \\
\hline Trägerschaft & Kommunale Beschäftigungsgesellschaft der Stadt Bielefeld (REGEmbH) \\
\hline Rechtsform & $\begin{array}{l}\text { Kooperationsprojekt der INITA (Institut für angewandte Psychologie, Transaktionsanalyse } \\
\text { und Erwachsenenbildung) gemeinnützige GmbH in Hannover und der REGEmbH } \\
\text { (Regionale Personalentwicklungsgesellschaft mbH) in Bielefeld. }\end{array}$ \\
\hline Finanzierungsquellen & $\begin{array}{l}\text { Die Kosten des Projekts werden letztendlich über den Sozialhilfeetat der Stadt Bielefeld } \\
\text { gedeckt. Zwischengeschaltet ist die REGE, welche die INITA als projektdurchführende } \\
\text { Institution bezahlt, die wiederum die Projektmitarbeiter vergütet. } \\
\text { Die REGE selbst finanziert sich aus Mitteln der Stadt Bielefeld (ca. 45 \%), des Landes } \\
\text { Nordrhein-Westfalen, des Bundes und der EU }\end{array}$ \\
\hline Personal & $\begin{array}{l}\text { Im Projekt sind elf Personen (Vollzeit- und Teilzeitkräfte) zuzüglich einiger Honorarkräfte } \\
\text { beschäftigt. } \\
\text { sechs Trainer (drei Vollzeit-, drei Teilzeitstellen), überwiegend Sozialpädagogen, ge- } \\
\text { schult in Teamentwicklung und Organisationsberatung } \\
\text { zwei Personen sind zuständig für die Projektrahmenleitung und Projektsteuerung } \\
\text { (Institutsleiter INITA, Bereichsleiter REGE) } \\
\text { eine Person ist zuständig für die Projektkoordination } \\
\text { eine Person ist zuständig für die Arbeitsvermittlung (8,5 Stunden monatlich) } \\
\text { eine Person ist zuständig für die Ablauforganisation und Betreuung (halbe Stelle) } \\
\text { mehrere Honorarkräfte für die Datenverarbeitung } \\
\text { Außerhalb des Projekts waren die wissenschaftliche Begleitung sowie alle zwei bis drei } \\
\text { Wochen drei Stunden lang eine Supervisorin tätig. }\end{array}$ \\
\hline Größe & $\begin{array}{l}61 \text { Teilnehmer begannen 1997 die drei Kurse des Pilotprojektes in Bielefeld. Von innen } \\
\text { schieden neun während der ersten acht Wochen (Potentialanalyse) aus. }\end{array}$ \\
\hline Aufgabenfelder & \\
\hline
\end{tabular}

${ }^{44}$ seit 01.01.1998: 1.000 Ressourcen - Qualifizierende Arbeitsvermittlung auf neuen Wegen

${ }^{45}$ Vgl.: Böckmann-Schewe, Lisa/Röhrig, Anne $(1997,159)$ 


\begin{tabular}{|c|c|}
\hline Kriterium & START Zeitarbeit NRW GmbH \\
\hline \multicolumn{2}{|l|}{ Angebote } \\
\hline Dauer der Beschäftigung & $\begin{array}{l}\text { achtmonatiges Programm aufgeteilt in drei Phasen: } \\
\text { 1. Potentialanalyse (acht Wochen, siehe Orientierung) } \\
\text { 2. Arbeitsvermittlung (drei Monate, siehe Vermittlung) } \\
\text { 3. Betreuung nach der Arbeitsaufnahme (drei Monate, siehe Betreuung) }\end{array}$ \\
\hline Entlohnung & $\begin{array}{l}\text { Die Kursteilnehmer des Projekts stehen bis zu einer eventuellen Arbeitsaufnahme im So- } \\
\text { zialhilfebezug. Zusätzlich erhalten sie während des Trainings eine Monatskarte des ÖPNV } \\
\text { sowie in einem bestimmten Rahmen bedarfsbezogene Aufwendungen für Bewerbungen } \\
\text { (bspw. entsprechende Kleidung für Vorstellungsgespräche). }\end{array}$ \\
\hline TeilzeitNollzeit & - \\
\hline Teilnehmergewinnung & $\begin{array}{l}\text { Die Teilnehmer des achtmonatigen Projektprogramms werden über eine Informationsver- } \\
\text { anstaltung gewonnen. Zu dieser Veranstaltung gelangen sie entweder: } \\
\text { - über das Sozialamt (Teilnahmezwang) oder durch } \\
\text { - Plakatwerbung (z.B. im OPNV). } \\
\text { Innerhalb der anderthalbstündigen Informationsveranstaltung stellen ein REGE-Berater } \\
\text { und ein Trainer Konzept und Ablauf des nächsten Kurses vor. } \\
\text { Die Bedingungen für eine Teilnahme an den drei Projektphasen sind: } \\
\text { - kein Leistungsbezug beim Arbeitsamt } \\
\text { - Arbeitserlaubnis in Deutschland } \\
\text { - keine gesundheitliche Beeinträchtigung (z.B. Drogenabhängigkeit) } \\
\text { - ausreichende Deutschkenntnisse } \\
\text { "Mit den nach der Information verbleibenden Interessentlnnen wurde in einem Einzelge- } \\
\text { spräch eine schriftliche Teilnahmevereinbarung geschlossen, die alle Leistungen und Er- } \\
\text { wartungen an die Teilnehmenden aufführ"46. Bisher war die Zahl der Interessenten in et- } \\
\text { wa gleich der Anzahl der verfügbaren Plätze, so daß noch keine Auswahlverfahren not- } \\
\text { wendig wurden. }\end{array}$ \\
\hline
\end{tabular}

${ }^{46}$ Kehler, Irene $(1998,9)$ 


\begin{tabular}{|c|c|}
\hline Kriterium & START Zeitarbeit NRW GmbH \\
\hline Beratung/Orientierung & $\begin{array}{l}\text { Beratung und Orientierung findet im Rahmen der ersten Phase statt, welche eine Poten- } \\
\text { tialanalyse in Form eines achtwöchigen Trainings mit Diagnoseverfahren beinhaltet. } \\
\text { - I.d.R. drei Trainer arbeiten - individuell beratend - mit durchschnittlich } 20 \text { Teilnehmern. } \\
\text { - Gearbeitet wird sowohl in Gesamt- als auch in Kleingruppenarbeitskreisen. } \\
\Rightarrow \text { Kennenlernen und Akzeptieren von: } \\
\text { - eigenen Stärken/Schwächen, } \\
\text { - evtl. brachliegenden Potentialen und } \\
\text { - verdrängten beruflichen Wünschen. } \\
\text { Folgende Schwerpunkte der Entwicklung und Analyse sind Bestandteil des achtwöchigen } \\
\text { Trainings: } \\
\text { - Grundmotivation } \\
\text { - soziales Verhalten (z.B. Feedbackregeln) } \\
\text { - Lernverhalten (Lernerfahrungen und -muster) } \\
\text { - Streßverhalten (Assessment Center) } \\
\text { - Berufsfeldanalyse } \\
\text { - biografische Analyse } \\
\text { - Arbeitsfeldanalyse } \\
\text { - Ressourcenanalyse } \\
\text { - Berufsorientierung } \\
\text { - Problemfeldanalyse } \\
\text { - Planungsverhalten } \\
\text { - Betreuungsverhalten } \\
\text { - Bewerberverhalten } \\
\text { Im Laufe dieser ersten Phase erstellen die jeweiligen Trainer für jedes Mitglied ihrer Klein- } \\
\text { gruppe insgesamt } 23 \text { Erhebungsbögen zu den oben genannten Schwerpunkten, welche }\end{array}$ \\
\hline
\end{tabular}




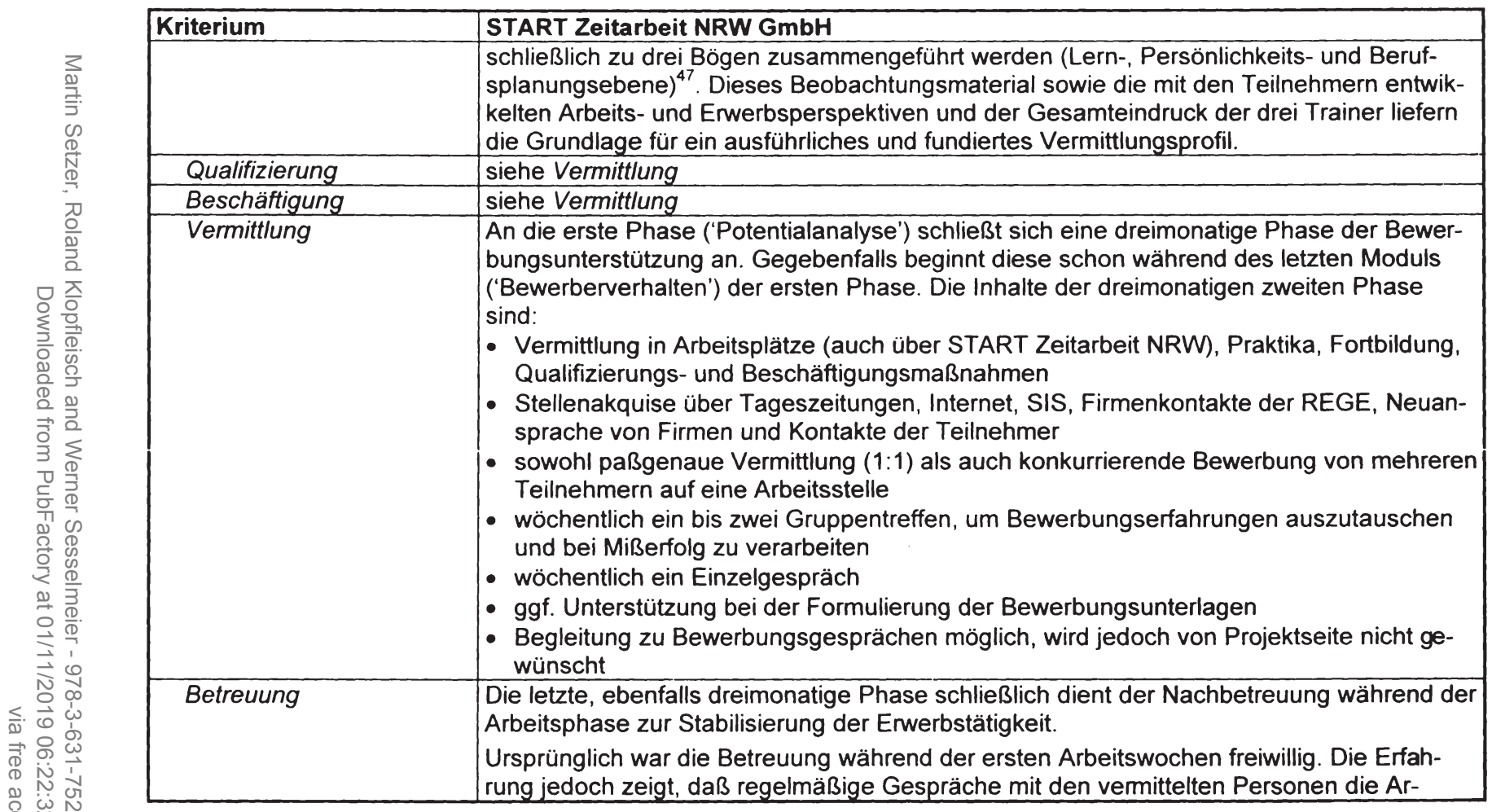

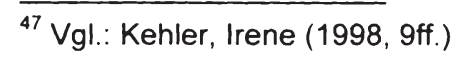




\begin{tabular}{|c|c|}
\hline Kriterium & START Zeitarbeit NRW GmbH \\
\hline & beitssituation stabilisieren und vorschnelle Abbrüche verhindern hilft. \\
\hline & $\begin{array}{l}\text { Die Kontakte mit den Arbeitgebern wurden sowohl durch die wissenschaftliche Begleitung } \\
\text { als auch durch das Projektteam sichergestellt. }\end{array}$ \\
\hline & $\begin{array}{l}\text { Die nicht in den Ersten Arbeitsmarkt vermittelten Teilnehmer, von denen ein Teil Fortbil- } \\
\text { dungs-, Qualifizierungs- oder Beschäftigungsmaßnahmen besuchen, haben ebenfalls die } \\
\text { Möglichkeit, Einzelgespräche zu vereinbaren. }\end{array}$ \\
\hline \multicolumn{2}{|l|}{ Erfolgskriterien } \\
\hline Erreichen der Zielgruppe & $\begin{array}{l}\text { Die Zielgruppe des Projektes besteht aus arbeitsfähigen Sozialhilfeempfängern aus allen } \\
\text { Bereichen und allen Altersklassen. } \\
\text { - Im ersten Halbjahr } 1997 \text { registrierte die REGE in Bielefeld } 1.254 \text { arbeitsfähige Sozialhil- } \\
\text { feempfänger. } \\
\text { - Die Anzahl von } 61 \text { Teilnehmern in den drei stattgefundenen Kursen entspricht einem } \\
\text { Erfassungssatz von } 4,9 \% \text { bezüglich der Gesamtmenge. } \\
\text { - Die Verteilung der Kursteilnehmer entspricht in etwa der Verteilung der Grundgesamt- } \\
\text { heit aller arbeitsfähigen Sozialhilfeempfänger. "Insgesamt ist von der Zusammenset- } \\
\text { zung der Kursteilnehmerlnnen nach den erfaßten Merkmalen kein positiver Effekt auf } \\
\text { die Vermittlungsergebnisse zu erwarten"48. }\end{array}$ \\
\hline \multicolumn{2}{|l|}{ Integrationsleistungen } \\
\hline Projektintegration & $\begin{array}{l}52 \text { von } 61 \text { Personen }(85,2 \%) \\
\text { Von den neun ausgeschiedenen Personen waren einige wegen Krankheit so oft abwe- } \\
\text { send, daß ein späterer Einstieg den Trainern nicht mehr möglich erschien. Ein anderer } \\
\text { Teil dieser Personen blieb zunächst ohne Angabe von Gründen dem Training fern und } \\
\text { lehnte auf Rückfrage eine weitere Teilnahme ab. Ein Teilnehmer konnte wegen Sucht- } \\
\text { problemen nicht im Training verbleiben. }\end{array}$ \\
\hline Qualifizierungsphase & 46 von 61 Personen $(75,4 \%)$ \\
\hline
\end{tabular}

${ }^{48}$ Kehler, Irene $(1998,19)$ 


\begin{tabular}{|c|c|c|}
\hline \multirow{6}{*}{ 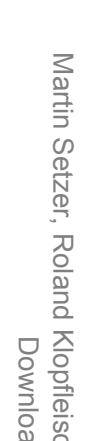 } & Kriterium & START Zeitarbeit NRW GmbH \\
\hline & & $\begin{array}{l}\text { Sechs Personen brachen nach der Trainigsphase ab. Davon drei wegen Angst vor dem } \\
\text { Schritt in die Arbeitswelt, zwei wegen vielfältiger sozialer Probleme und einer wegen Exi- } \\
\text { stenzgründung. }\end{array}$ \\
\hline & Arbeitsmarktintegration & $\begin{array}{l}22 \text { von } 61 \text { Personen }(36,1 \%) \text { konnten in den Ersten Arbeitsmarkt bzw. in betriebliche } \\
\text { Ausbildungen vermittelt werden. }\end{array}$ \\
\hline & Beschäftigungswirkungen & Erste Daten werden Ende Januar 1999 veröffentlicht. \\
\hline & Kosten & nicht errechenbar \\
\hline & Sonstiges & $\begin{array}{l}\text { Seit Mitte } 1998 \text { steht der Know-how-Transfer im Mittelpunkt der Projektarbeit. Die Pro- } \\
\text { jektangebote wurden in Module untergliedert, welche einzeln zu belegen sind. Als neues } \\
\text { Modul existiert die Weiterbildung von kommunalen Arbeitsvermittlern in der Vermittlungs- } \\
\text { methode des Projektes. }\end{array}$ \\
\hline
\end{tabular}




\begin{tabular}{|c|c|}
\hline Kriterium & Internationaler Bund Darmstadt \\
\hline \multicolumn{2}{|c|}{ Projektstrukturdaten } \\
\hline Trägerschaft & freie Trägerschaft \\
\hline Rechtsform & gemeinnütziger eingetragener Verein \\
\hline Finanzierungsquellen & $\begin{array}{l}\text { - Mitgliedsbeiträge } \\
\text { - Spenden } \\
\text { - Zuschüsse der öffentlichen Hand } \\
\text { - Entgelte für Dienstleistungen im Auftrag von Betrieben der gewerblichen Wirtschaft } \\
\text { - Das Beschäftigungsprogramm "Renovierung und Recycling" wird von der Stadt Darm- } \\
\text { stadt finanziert. }\end{array}$ \\
\hline Personal & $\begin{array}{l}\text { - Stammpersonal } 250 \text { Personen, davon etwa } 25 \text { bis } 30 \text { in der Beschäftigungsförderung } \\
\text { - Auf Stellen des Zweiten Arbeitsmarktes arbeiten rund } 100 \text { Personen, davon etwa ein } \\
\text { Drittel in der Beschäftigungsförderung. } \\
\text { - Bundesweit arbeiten über } 11.000 \text { Personen in über } 680 \text { Einrichtungen. }\end{array}$ \\
\hline Größe & $\begin{array}{l}\text { Die unten genannten Projekte (siehe: Aufgabenfelder) beherbergen insgesamt } 387 \text { Plätze, } \\
\text { davon allerdings alleine } 320 \text { Plätze in den Orientierungslehrgängen }{ }^{49} \text {. }\end{array}$ \\
\hline Aufgabenfelder & $\begin{array}{l}\text { - Projekt 'Werkstatt Arbeit und Umwelt' } \\
\text { (Renovieren von Darmstädter Schulen) } \\
\text { - Projekt 'Renovieren und Recyclen' } \\
\text { Renovieren von Wohnungen und öffentlichen Einrichtungen) } \\
\text { - Projekt 'E-Recycling' } \\
\text { (Sortieren und Demontieren von Elektronikschrott und Elektrogeräten) } \\
\text { - Projekt 'Orientierungslehrgänge/Erstberatung' } \\
\text { (siehe: Beratung und Orientierung) } \\
\text { - Projekt 'Second-Hand-Kaufhaus' } \\
\text { (Verkauf, Logisitik, Buchhaltung, Reperatur/Aufbereitung) }\end{array}$ \\
\hline ngebote & \\
\hline
\end{tabular}

${ }^{49}$ Vgl.: Stadt Darmstadt (1998, Anlage 8ff.) 


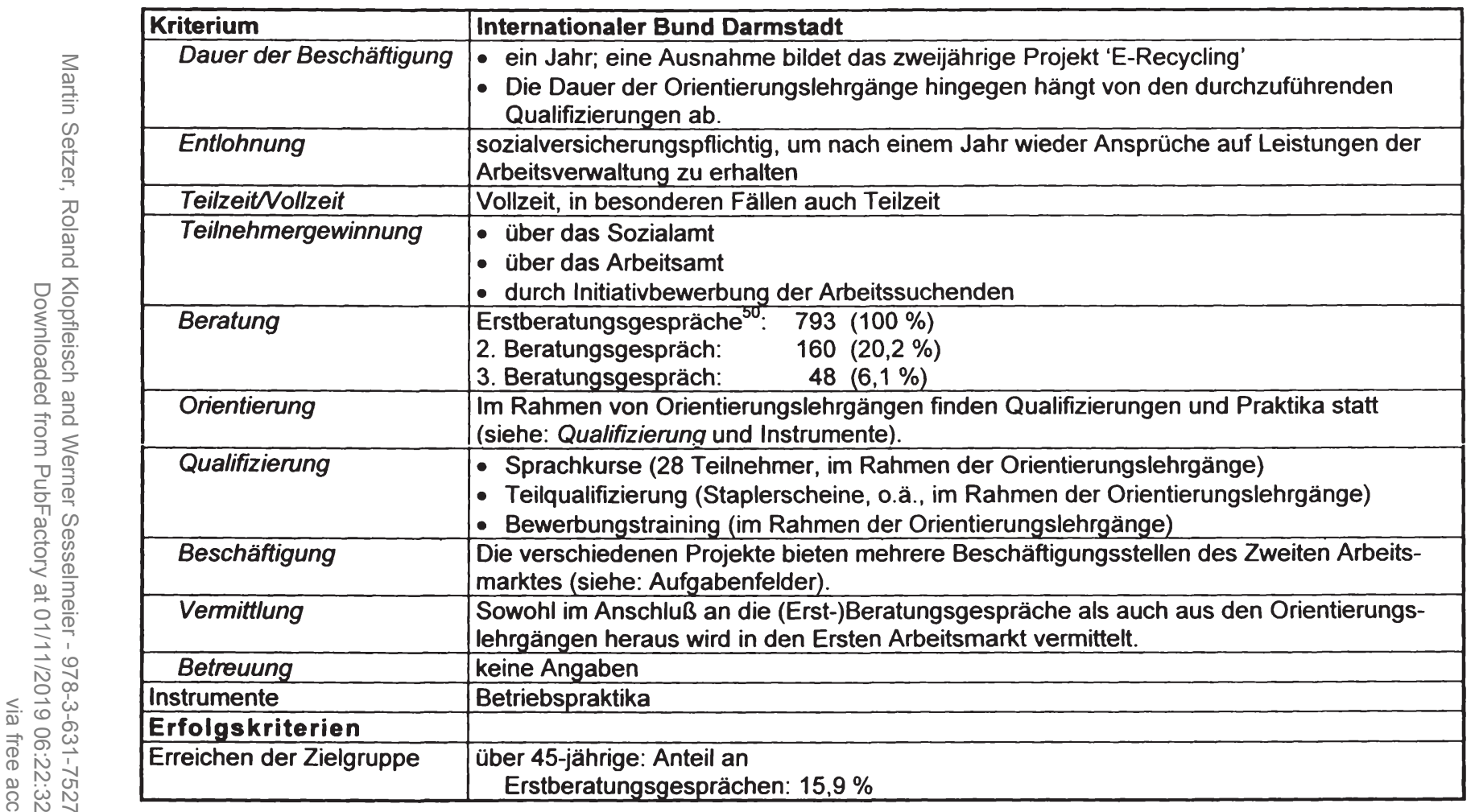

${ }^{50}$ Alle nachfolgenden Daten beziehen sich auf den Zeitraum von Januar bis August 1998, Internationaler Bund Darmstadt 


\begin{tabular}{|c|c|}
\hline Kriterium & Internationaler Bund Darmstadt \\
\hline & $\begin{array}{l}\text { Orientierungslehrgängen: } 15,8 \% \\
\text { Spätaussiedler: Anteil an } \\
\text { Erstberatungsgesprächen: } 12,9 \% \\
\text { Orientierungslehrgängen: } 4,6 \% \\
\text { Alleinerziehende: Anteil an } \\
\text { Erstberatungsgesprächen: } 7,2 \% \\
\text { Orientierungslehrgängen: } 9,9 \% \\
\end{array}$ \\
\hline \multicolumn{2}{|l|}{ Integrationsleistungen } \\
\hline Projektintegration & - \\
\hline Qualifizierungsphase & - \\
\hline Arbeitsmarktintegration & $\begin{array}{l}793 \text { Teilnehmer an einem Erstberatungsgespräch, davon vermittelt in: } \\
\text { Ersten Arbeitsmarkt: } 33(4,2 \%) \\
\text { Zweiten Arbeitsmarkt: } 10(1,3 \%) \\
152 \text { Teilnehmer an einem Orientierungslehrgang, davon vermittelt in: } \\
\text { Ersten Árbeitsmarkt: } \overline{2} \overline{6}(1 \overline{7}, 1 \%) \\
\text { Zweiten Arbeitsmarkt: } 16(10,5 \%) \\
\text { Praktikum: } 84(55,3 \%)\end{array}$ \\
\hline Beschäftigungswirkungen & keine Angaben \\
\hline Kosten & keine Angaben \\
\hline
\end{tabular}




\title{
SOZIALÖKONOMISCHE SCHRIFTEN
}

\author{
Herausgegeben von Professor Dr. Dr. h.c. Bert Rürup
}

Band 1 Marietta Jass: Erfolgskontrolle des Abwasserabgabengesetzes. Ein Konzept zur Erfassung der Gesetzeswirkungen verbunden mit einer empirischen Untersuchung in der $\mathrm{Pa}$ pierindustrie. 1990.

Band 2 Frank Schulz-Nieswandt: Stationäre Altenpflege und "Pflegenotstand" in der Bundesrepublik Deutschland. 1990.

Band 3 Helmut Böhme, Alois Peressin (Hrsg.): Sozialraum Europa. Die soziale Dimension des Europäischen Binnenmarktes. 1990.

Band 4 Stephan Ruß: Telekommunikation als Standortfaktor für Klein- und Mittelbetriebe. Telekommunikative Entwicklungstendenzen und regionale Wirtschaftspolitik am Beispiel Hessen. 1991.

Band 5 Reinhard Grünewald: Tertiärisierungsdefizite im Industrieland Bundesrepublik Deutschland. Nachweis und politische Konsequenzen. 1992.

Band 6 Bert Rürup, Uwe H. Schneider (Hrsg.): Umwelt und Technik in den Europäischen Gemeinschaften. Teil I: Die grenzüberschreitende Entsorgung von Abfällen. Bearbeitet von: Thomas Kemmler, Thomas Steinbacher. 1993.

Band 7 Mihai Nedelea: Erfordernisse und Möglichkeiten einer wachstumsorientierten Steuerpolitik in Rumänien. Dargestellt am Beispiel der Textil- und Bekleidungsindustrie. 1995.

Band 8 Andreas Schade: Ganzjährige Beschäftigung in der Bauwirtschaft - Eine Wirkungsanalyse. Analyse und Ansätze für eine Reform der Winterbauförderung. 1995.

Band 9 Frank Schulz-Nieswandt: Ökonomik der Transformation als wirtschafts- und gesellschaftspolitisches Problem. Eine Einführung aus wirtschaftsanthropologischer Sicht. 1996.

Band 10 Werner Sesselmeier / Roland Klopfleisch / Martin Setzer: Mehr Beschäftigung durch eine Negative Einkommensteuer. Zur beschäftigungspolitischen Effektivität und Effizienz eines integrierten Steuer- und Transfersystems. 1996.

Band 11 Sylvia Liebler: Der Einfluß der Unabhängigkeit von Notenbanken auf die Stabilität des Geldwertes. 1996.

Band 12 Werner Sesselmeier: Einkommenstransfers als Instrumente der Beschäftigungspolitik. Negative Einkommensteuer und Lohnsubventionen im Lichte moderner Arbeitsmarkttheorien und der Neuen Institutionenökonomik. 1997.

Band 13 Stefan Lorenz: Der Zusammenhang von Arbeitsgestaltung und Erwerbsleben unter besonderer Berücksichtigung der Erwerbstätigkeiten von Frauen und Älteren. 1997.

Band 14 Volker Ehrlich: Arbeitslosigkeit und zweiter Arbeitsmarkt. Theoretische Grundlagen, Probleme und Erfahrungen. 1997.

Band 15 Philipp Hartmann: Grenzen der Versicherbarkeit. Private Arbeitslosenversicherung. 1998.

Band 16 Martin Setzer / Roland Klopfleisch / Werner Sesselmeier: Langzeitarbeitslose und Erster Arbeitsmarkt. Eine kombinierte Strategie zur Erhöhung der Wiederbeschäftigungschancen. 1999. 


\section{Alfred Stiassny}

\section{Persistente Arbeitslosigkeit}

Frankfurt/M., Berlin, Bern, New York, Paris, Wien, 1998.

284 S., 7 Tab., 41 Graf.

ISBN 3-631-32445-6 · br. DM 89.-*

Seit den letzten 20 Jahren sind fast alle europäischen Staaten mit einer ständig steigenden Arbeitslosigkeit konfrontiert. In diesem Buch werden einige Erklärungsversuche für dieses Phänomen näher betrachtet.

O) Besonderer Wert wird auf das Lohnsetzungsverhalten der Gewerkschaften und auf einen möglichen Beitrag von Effizienzlohntheorien gelegt.

Außerdem wird der Einfluß von Gütermarktimperfektionen untersucht und auf diese Weise Ansätze der sogenannten „New Keynesian Economics" mit modernen Arbeitsmarkttheorien kombiniert.

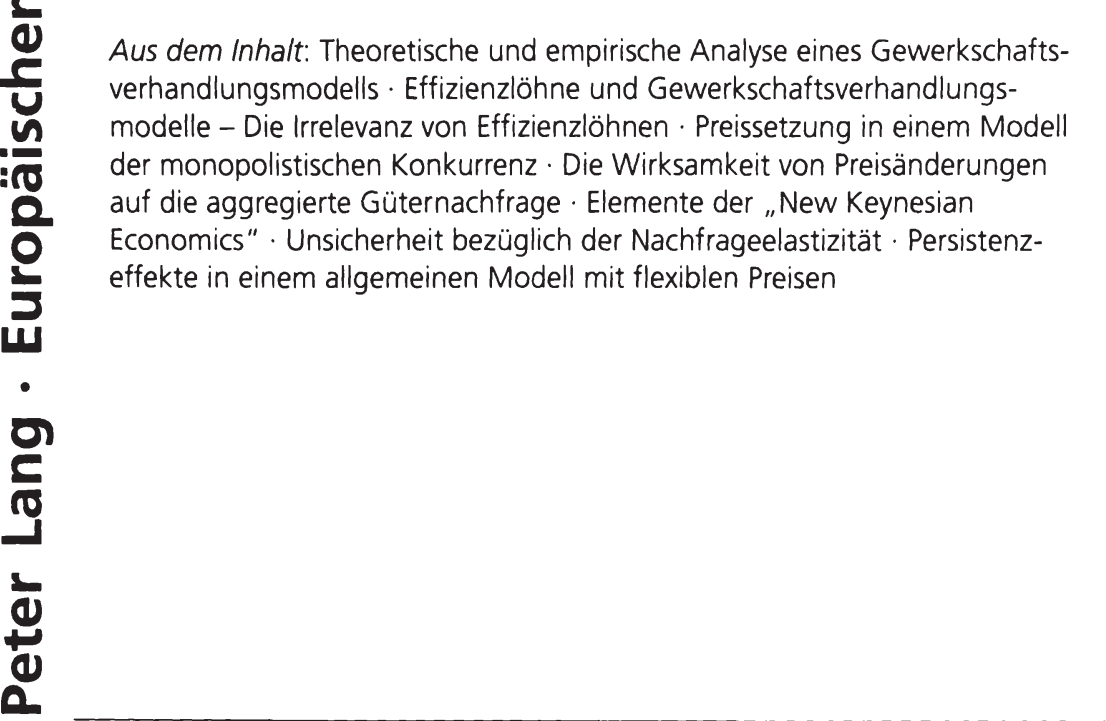

Frankfurt/M - Berlin - Bern - New York - Paris - Wien

Auslieferung: Verlag Peter Lang AG

Jupiterstr. 15, CH-3000 Bern 15

Telefax (004131) 9402131

*inklusive Mehrwertsteuer

Preisänderungen vorbehalten

Martin Setzer, Roland Klopfleisch and Werner Sesselmeier - 978-3-631-75277-7 